\title{
GENERATING MAXIMAL SUBGROUPS OF FINITE ALMOST SIMPLE GROUPS
}

\author{
ANDREA LUCCHINI ${ }^{1}$, CLAUDE MARION ${ }^{2}$ and GARETH TRACEY ${ }^{3}$ \\ ${ }^{1}$ Dipartimento di Matematica Tullio Levi-Civita, Università degli Studi di Padova, \\ 35121-I Padova, Italy; \\ email: lucchini@math.unipd.it \\ 2 CMUP, Departamento de Matemática, Universidade do Porto, 4169-007 Porto, Portugal; \\ email: claude.marion@fc.up.pt \\ ${ }^{3}$ Department of Mathematical Sciences, University of Bath, Bath BA2 7AY, UK; \\ email: gmt29@bath.ac.uk
}

Received 28 December 2018; accepted 4 November 2019

\begin{abstract}
For a finite group $G$, let $d(G)$ denote the minimal number of elements required to generate $G$. In this paper, we prove sharp upper bounds on $d(H)$ whenever $H$ is a maximal subgroup of a finite almost simple group. In particular, we show that $d(H) \leqslant 5$ and that $d(H) \geqslant 4$ if and only if $H$ occurs in a known list. This improves a result of Burness, Liebeck and Shalev. The method involves the theory of crowns in finite groups.
\end{abstract}

2010 Mathematics Subject Classification: 20F05 (primary); 20D05, 20E28 (secondary)

\section{Introduction}

For a finite group $G$, let $d(G)$ denote the minimal number of elements required to generate $G$. It is well known that if $G$ is a nonabelian finite simple group, then $d(G)=2$; see $[2,43,47]$. More generally, if $G$ is almost simple with simple socle $G_{0}$ (that is, $G_{0} \leqslant G \leqslant \operatorname{Aut}\left(G_{0}\right)$ with $G_{0}$ a nonabelian finite simple group), then, by [17], $d(G)=\max \left\{2, d\left(G / G_{0}\right)\right\} \leqslant 3$.

In this paper, we consider the corresponding result for maximal subgroups $H$ of almost simple groups $G$. In [10], Burness, Liebeck and Shalev prove that like $G$, $H$ can be generated by a bounded number of elements. More precisely, they show

(c) The Author(s) 2020. This is an Open Access article, distributed under the terms of the Creative Commons Attribution licence (http://creativecommons.org/licenses/by/4.0/), which permits unrestricted re-use, distribution, and reproduction in any medium, provided the original work is properly cited. 
that $d(H) \leqslant 6$. Furthermore, they prove the sharp upper bounds $d(H) \leqslant 4$ if $G$ is simple or if $\operatorname{soc}(G)$ is an alternating group, and $d(H) \leqslant 3$ if $\operatorname{soc}(G)$ is a sporadic group. They also speculate that the bound $d(H) \leqslant 4$ may hold in the general case. We use the theory of crowns in finite groups to investigate this problem. We prove that $d(H) \leqslant 5$ and that $d(H) \geqslant 4$ if and only if $H$ occurs in a known list (see Theorem 1(ii)). In particular, we prove that $d(H) \leqslant 3$ when $G$ is an almost simple group with socle an exceptional group of Lie type.

Before precisely stating our theorem, we require the following notation and terminology. Throughout the paper, we will, for the most part, use the notation from [31] for group names. In particular, $Z_{n}$ denotes a cyclic group of order $n$, although we do use $n$ instead when there is no ambiguity. Similarly, we will write $p^{n}$ in place of $\left(Z_{p}\right)^{n}$ to denote an elementary abelian group of order $p^{n}$, for a prime $p$. We will denote the alternating and symmetric groups by $\mathrm{Alt}_{n}$ and $\mathrm{Sym}_{n}$, respectively. The notation $\operatorname{Frat}(G)$ will denote the Frattini subgroup of the group $G$, while $\operatorname{soc}(G)$ denotes the socle of $G$. We will write $\operatorname{Aut}(G)$ and $\operatorname{Out}(G)$ for the automorphism and outer automorphism group of $G$, respectively. With core ${ }_{G}(M)$, we will denote the normal core of $M$ in $G$.

The statement of our main result (Theorem 1) requires a preliminary discussion of finite classical groups and their maximal subgroups. Let $G$ be a finite classical group with natural module $V$. The main theorem on the subgroup structure of classical groups is due to Aschbacher. In [1], eight collections of subgroups of $G$ are defined, labelled $\mathcal{C}_{i}$ for $1 \leqslant i \leqslant 8$, and it is shown that if $H$ is a maximal subgroup of $G$, then either $H$ is contained in one of these natural subgroup collections or it belongs to a family of almost simple subgroups that act irreducibly on $V$. Sometimes in the literature, $\mathcal{S}$ is used to denote this additional subgroup collection, but, here, we use the definitions from [31], which differ slightly from [1], and we denote by $\mathcal{C}_{9}$ this class.

We also subdivide each class $\mathcal{C}_{i}$ into types as in [31]. To state our main theorem, we require a description of the classes $\mathcal{C}_{1}, \mathcal{C}_{2}, \mathcal{C}_{4}$ and $\mathcal{C}_{7}$ and the types therein. This will be given in the paragraphs below.

Let $G$ be one of the groups $\operatorname{SL}_{n}(q), \operatorname{SU}_{n}(q), \operatorname{Sp}_{n}(q)$ or $\Omega_{n}^{\epsilon}(q)(\epsilon \in\{\circ, \pm\})$. Let $H$ be a subgroup of $G$, and let $V$ denote the natural $G$-module. We say that $H$ is in class $\mathcal{C}_{1}$ if $H=\operatorname{Stab}_{G}(W)$ or $H=\operatorname{Stab}_{G}(W, U)$, for nondegenerate, totally singular or nonsingular subspaces $W$ and $U$ of $V$, as in [31, Table 4.1.A]. The types of $H$ are also given in [31, Table 4.1.A]. In particular, we say that a subgroup $H \in \mathcal{C}_{1}$ is parabolic if $H$ contains a $G$-conjugate of a Borel subgroup of $G$. In this case, either $H$ stabilizes a totally singular subspace $W \subset V$ of dimension $m \leqslant n / 2$ or $G=\operatorname{SL}_{n}(q)$ and $H$ stabilizes subspaces $W$ and $U$ with $W \subset U$ and $\operatorname{dim} W=m \leqslant n / 2, \operatorname{dim} U=n-m$. Such parabolic subgroups are said to have type $P_{m}$ and type $P_{m, n-m}$, respectively. 
We say that $H$ is in class $\mathcal{C}_{2}$ if $H=\operatorname{Stab}_{G}\left(\left\{V_{1}, \ldots, V_{t}\right\}\right)$, where $V=V_{1} \oplus \cdots \oplus$ $V_{t}$ is a direct sum decomposition of $V$ consisting of subspaces $V_{i}$ of common dimension $m:=n / t$. Furthermore, the $V_{i}$ are either nondegenerate for all $i$ or totally singular for all $i$. The types of $H$ are $\operatorname{GL}_{m}(q)$ 2 $\operatorname{Sym}_{t}, \operatorname{GU}_{m}(q)$ 2 $\operatorname{Sym}_{t}$, $\operatorname{Sp}_{m}(q)$ 2 $\operatorname{Sym}_{t}, \mathrm{O}_{m}^{\epsilon}(q)$ 2 $\operatorname{Sym}_{t}, \mathrm{GL}_{n / 2}\left(q^{u}\right) .2$ and $\Omega_{n / 2}(q)^{2}$ (see [31, Table 4.2.A]).

We now describe the classes $\mathcal{C}_{4}$ and $\mathcal{C}_{7}$. For a decomposition $n=\prod_{i=1}^{t} n_{i}$, we can view the vector space $V$ as a tensor product $V=V_{1} \otimes \cdots \otimes V_{t}$, where $\operatorname{dim} V_{i}=$ $n_{i}$. Write $G_{i}$ for the stabilizer in $G$ of the set $0 \otimes \cdots \otimes V_{i} \otimes \cdots \otimes 0$ of pure tensors with the only nonzero element occurring in the $i$ th position. Also, set $K:=\bigcap_{i=1}^{t} G_{i}$.

The group $H$ is in class $\mathcal{C}_{4}$ if $H$ is the stabilizer of a tensor decomposition $V=V_{1} \otimes V_{2}$ of $V$ with the property that the induced actions of $H \cap G_{i}$ on $V_{i}$ preserves a certain form $f_{i}$ on $V_{i}$, given in [31, Table 4.4.A]. In particular, $\left(V_{1}, f_{1}\right)$ and $\left(V_{2}, f_{2}\right)$ are not similar; so $H=K$. The types of $H$ are $\mathrm{GL}_{n_{1}}(q) \otimes \mathrm{GL}_{n_{2}}(q)$, $\mathrm{GU}_{n_{1}}(q) \otimes \mathrm{GU}_{n_{2}}(q), \mathrm{Sp}_{n_{1}}(q) \otimes \mathrm{O}_{n_{2}}^{\epsilon}(q), \operatorname{Sp}_{n_{1}}(q) \otimes \operatorname{Sp}_{n_{2}}(q)$ and $\Omega_{n_{1}}^{\epsilon_{1}}(q) \otimes \Omega_{n_{2}}^{\epsilon_{2}}(q)$ (see [31, Table 4.4.A]).

Finally, $H$ is in class $\mathcal{C}_{7}$ if $H$ is the stabilizer of a tensor decomposition $V=$ $V_{1} \otimes \cdots \otimes V_{t}$, where the induced action of $H \cap G_{i}$ on $V_{i}$ preserves a certain form $f_{i}$ on $V_{i}$, given in [31, Table 4.7.A]. In this case, $\left(V_{1}, f_{1}\right)$ is similar to $\left(V_{i}\right.$, $f_{i}$ ) for each $i, K<H$, and $H / K$ transitively permutes the set $\left\{V_{1}, \ldots, V_{t}\right\}$. The

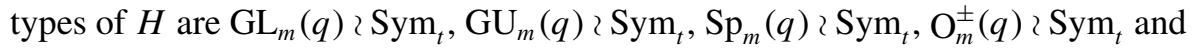
$\mathrm{O}_{m}(q)$ 2 $\operatorname{Sym}_{t}$ (see [31, Table 4.7.A]).

The other classes (and their associated types) are defined in [31, Ch. 4] for $1 \leqslant i \leqslant 8$ (see [31, Section 4.i] for information about class $\mathcal{C}_{i}$ ).

Finally, if $G$ is an almost simple classical group and $H$ is a maximal subgroup of $G$, then $H$ is said to be of class $\mathcal{C}_{i}$ and type $\mathcal{T}$ if $H \cap \operatorname{soc}(G)$ lifts to a group of class $\mathcal{C}_{i}$ and type $\mathcal{T}$ in the associated matrix group, as defined above.

Let $G$ be an almost simple classical group with socle $G_{0}$, and assume that either $G_{0}=\mathrm{L}_{n}(q)$ with $n \geqslant 3$ or

$G_{0}=\mathrm{P} \Omega_{n}^{\epsilon}(q), q$ is odd, $\epsilon \in\{+,-\}$, the associated quadratic form has square discriminant and $G$ is contained in $\mathrm{P} \Gamma \mathrm{O}_{n}^{\epsilon}(q)$.

Note that the final condition in (1.1) is vacuously true unless $(n, \epsilon)=(8,+)$. Write $q=p^{f}$, with $p$ prime. We now make some remarks about subgroups of $\operatorname{Out}\left(G_{0}\right)$ containing $G$ in these cases. Our reference is [31, Ch. 2]. Note first that we have $\operatorname{Out}\left(\mathrm{L}_{n}(q)\right)=\langle\delta, \phi, g\rangle$, subject to the relations

$$
\delta^{(n, q-1)}=\phi^{f}=g^{2}=1 \quad \text { and } \quad \delta^{\phi}=\delta^{p}, \delta^{g}=\delta^{-1}, \phi^{g}=\phi .
$$


Also, we have $\mathrm{P} \mathrm{O}_{n}^{\epsilon}(q)=\langle r, s, \delta, \phi, g\rangle$, with $r=s$ if the discriminant of the associated quadratic form is nonsquare. The relations are

$\delta^{2(2, n / 2-1)}=\phi^{f}=r^{2}=s^{2}=1, \quad \delta^{\phi}=\delta^{(-1)^{(n(p-1) / 4)}}, \quad r^{\delta}=s, s^{\delta}=r, \phi^{r}=\phi^{s}=\phi$

again by [31, Ch. 2]. Finally, if $G_{0}=\mathrm{P} \Omega_{n}^{-}(q)$ with square discriminant, then $\operatorname{Out}\left(G_{0}\right)=\langle r, s, \delta, \phi, g\rangle$, subject to the relations

$$
r^{2}=s^{2}=\delta^{2}=\phi^{f}=1 \quad \text { and } \quad \delta^{\phi}=\delta, r^{\delta}=s, s^{\delta}=r, \phi^{r}=\phi^{s}=\phi .
$$

Set $D:=\langle\delta\rangle$ in the case $G_{0}=\mathrm{L}_{n}(q)$ with $n \geqslant 3$, and $D:=\langle r, s, \delta\rangle$ in the case at (1.1). Also, set $F:=\langle\phi\rangle$ in each case. For $E \in\{D, F\}$, we then say that $(E)_{1}$ holds if $(G / \operatorname{soc}(G)) \cap E$ has even order and that $(E)_{0}$ holds otherwise. If we are in case $G_{0}=\mathrm{L}_{n}(q)$ and $G / G_{0}$ projects nontrivially onto $\operatorname{Out}\left(G_{0}\right) /\langle D, F\rangle$, then we will say that $(\gamma)$ holds. If we are in the case at $(1.1)$, and $\left(G / G_{0}\right) \cap D \cong 2^{2}$ or $D_{8}$, then we will say that $(D)_{2}$ holds. Finally, we will say that $(\star)$ holds if

(a) $\operatorname{soc}(G)=\mathrm{L}_{n}(q)$ with $n \geqslant 3$, and $(D)_{1},(F)_{1}$ and $(\gamma)$ hold or

(b) (1.1) holds, and $(D)_{2}$ and $(F)_{1}$ hold.

We remark that $d\left(G / G_{0}\right) \leqslant 3$ with equality if and only if $\operatorname{soc}(G) \in\left\{\mathrm{L}_{n}(q)\right.$, $\left.\mathrm{P} \Omega_{n}^{ \pm}(q)\right\}$ and $(\star)$ holds (see Proposition 3.12 and Lemma 6.4).

THEOREM 1. Let $G$ be a finite almost simple group with socle $G_{0}$, and let $H$ be a maximal subgroup of $G$. The following assertions hold:

(i) $d(H) \leqslant 5$.

(ii) $d(H) \geqslant 4$ if and only if one of the following holds, with $H$ given up to conjugation in $\operatorname{Aut}\left(G_{0}\right)$ :

(a) The socle of $G$ is an alternating group of degree $n$; $G \in\left\{\mathrm{Alt}_{n}, \mathrm{Sym}_{n}\right\}$; $H=\left(T^{k} .\left(\operatorname{Out}(T) \times \operatorname{Sym}_{k}\right)\right) \cap G$ is of diagonal type (that is, $n=|T|^{k-1}$, where $T$ is nonabelian simple and $k>1) ; \operatorname{Sym}_{k} \leqslant H ; d(\operatorname{Aut}(T) \cap$ $H)=3$. In this case, $d(H)=4$.

(b) The socle of $G$ is of classical type, with field of definition $\mathbb{F}_{q}$ and natural module of dimension $n$, and $(G, H)$ is one of the pairs listed in Table 1.

In particular, $d(H) \geqslant 4$ if and only if $H$ has an elementary abelian factor group of order $2^{d(H)}$.

Note that the conditions listed in the fourth column of Table 1 are in addition to all the relevant conditions in [9] and [31] that are needed for the existence and maximality of $H$ in $G$. We also remark that semicolons in the table mean 'and'. 
Table 1. The exceptional cases $(G, H)$ in part (ii) of Theorem 1 .

\begin{tabular}{|c|c|c|c|c|}
\hline$G_{0}$ & Class & Type & Conditions & $d(H)$ \\
\hline $\mathrm{L}_{n}(q)$ & $\mathcal{C}_{1}$ & $P_{m, n-m}$ & $m$ and $n-m$ are even; $(\star)$ holds & 4 \\
\hline $\mathrm{L}_{n}(q)$ & $\mathcal{C}_{2}$ & $\mathrm{GL}_{m}(q) \prec \mathrm{Sym}_{t}$ & $(\star)$ holds & 4 \\
\hline $\mathrm{L}_{n}(q)$ & $\mathcal{C}_{4}$ & $\mathrm{GL}_{n_{1}}(q) \otimes \mathrm{GL}_{n_{2}}(q)$ & $\begin{array}{l}n_{1} \text { and } n_{2} \text { are even; } q \text { is odd; } \\
d(G\langle\delta\rangle /\langle\delta\rangle)=2\end{array}$ & 4 \\
\hline $\mathrm{L}_{n}(q)$ & $\mathcal{C}_{7}$ & $\mathrm{GL}_{m}(q)$ \ $\mathrm{Sym}_{t}$ & $\begin{array}{l}(\star) \text { holds and either } t>2 \text { or } t=2 \text { and } \\
\text { either } m \neq \equiv 2(\bmod 4) \text { or } q \neq \equiv 3(\bmod \\
\text { 4) }\end{array}$ & 4 \\
\hline $\mathrm{P} \Omega_{n}^{ \pm}(q)$ & $\mathcal{C}_{1}$ & $\mathrm{O}_{m}^{\epsilon_{1}}(q) \oplus \mathrm{O}_{n-m}^{\epsilon_{2}}(q)$ & $\begin{array}{l}q \text { is odd; both } Q_{i} \text { have nonsquare } \\
\text { discriminant; }(\star) \text { holds; }\left(\epsilon_{1}, \epsilon_{2}\right) \neq \\
(-,-)\end{array}$ & 4 \\
\hline $\mathrm{P} \Omega_{n}^{ \pm}(q)$ & $\mathcal{C}_{1}$ & $\mathrm{O}_{m}^{\epsilon_{1}}(q) \oplus \mathrm{O}_{n-m}^{\epsilon_{2}}(q)$ & $\begin{array}{l}q \text { is odd; } Q_{2} \text { has square discriminant; } \\
(D)_{i} \text { and }(F)_{j} \text { hold with } i+j \geqslant 2\end{array}$ & $2+i+j$ \\
\hline $\mathrm{P} \Omega_{n}^{ \pm}(q)$ & $\mathcal{C}_{2}$ & $\mathrm{O}_{m}(q) \prec \mathrm{Sym}_{t}$ & $\begin{array}{l}m q \text { is odd; }(\star) \text { holds and either } m>1 \\
\text { or } m=1 \text { and } q \equiv \pm 1(\bmod 8)\end{array}$ & 4 \\
\hline $\mathrm{P} \Omega_{n}^{+}(q)$ & $\mathcal{C}_{4}$ & $\mathrm{O}_{n_{1}}^{\epsilon_{1}}(q) \otimes \mathrm{O}_{n_{2}}(q)$ & $\begin{array}{l}q \text { is odd; } n_{1} \text { is even; } n_{2} \text { is odd; }(\star) \\
\text { holds; } Q_{1} \text { has square discriminant if } \\
\epsilon_{1}=-\end{array}$ & 4 \\
\hline $\mathrm{P} \Omega_{n}^{+}(q)$ & $\mathcal{C}_{4}$ & $\mathrm{O}_{n_{1}}^{ \pm}(q) \otimes \mathrm{O}_{n_{2}}^{ \pm}(q)$ & $\begin{array}{l}q \text { is odd; each } Q_{i} \text { has square } \\
\text { discriminant; }(D)_{0} \text { and }(F)_{i} \text { hold }\end{array}$ & $4+i$ \\
\hline $\mathrm{P} \Omega_{n}^{+}(q)$ & $\mathcal{C}_{4}$ & $\mathrm{O}_{n_{1}}^{ \pm}(q) \otimes \mathrm{O}_{n_{2}}^{ \pm}(q)$ & $\begin{array}{l}q \text { is odd; each } Q_{i} \text { has square } \\
\text { discriminant; }(D)_{1} \text { and }(F)_{1} \text { hold }\end{array}$ & 4 \\
\hline $\mathrm{P} \Omega_{n}^{+}(q)$ & $\mathcal{C}_{4}$ & $\mathrm{O}_{n_{1}}^{\epsilon_{1}}(q) \otimes \mathrm{O}_{n_{2}}^{\epsilon_{2}}(q)$ & $\begin{array}{l}q \text { is odd; } \epsilon_{i} \in\{ \pm\} ; \epsilon_{i}=+ \text { if } D\left(Q_{i}\right) \\
\text { is nonsquare; the } Q_{i} \text { have distinct } \\
\text { discriminants; }(D)_{1} \text { holds }\end{array}$ & 4 \\
\hline $\mathrm{P} \Omega_{n}^{+}(q)$ & $\mathcal{C}_{4}$ & $\mathrm{O}_{n_{1}}^{+}(q) \otimes \mathrm{O}_{n_{2}}^{-}(q)$ & $\begin{array}{l}q \text { is odd; each } Q_{i} \text { has nonsquare } \\
\text { discriminant; }(D)_{1} \text { and }(F)_{1} \text { hold }\end{array}$ & 4 \\
\hline $\mathrm{P} \Omega_{n}^{+}(q)$ & $\mathcal{C}_{4}$ & $\mathrm{O}_{n_{1}}^{+}(q) \otimes \mathrm{O}_{n_{2}}^{+}(q)$ & $\begin{array}{l}q \text { is odd; each } Q_{i} \text { has nonsquare } \\
\text { discriminant; }(D)_{0} \text { and }(F)_{1} \text { hold }\end{array}$ & 4 \\
\hline $\mathrm{P} \Omega_{n}^{+}(q)$ & $\mathcal{C}_{4}$ & $\mathrm{O}_{n_{1}}^{+}(q) \otimes \mathrm{O}_{n_{2}}^{+}(q)$ & $\begin{array}{l}q \text { is odd; each } Q_{i} \text { has nonsquare } \\
\text { discriminant; }(D)_{1} \text { and }(F)_{i} \text { hold }\end{array}$ & $4+i$ \\
\hline $\mathrm{P} \Omega_{n}^{+}(q)$ & $\mathcal{C}_{7}$ & $\mathrm{O}_{m}^{\epsilon}(q)$ ? $\mathrm{Sym}_{2}$ & $(\star)$ holds; $m \equiv 0(\bmod 4)$ & 4 \\
\hline $\mathrm{P} \Omega_{n}^{+}(q)$ & $\mathcal{C}_{7}$ & $\mathrm{O}_{m}^{\epsilon}(q) \geq \mathrm{Sym}_{3}$ & $\begin{array}{l}(\star) \text { holds and either } m \not \equiv 2(\bmod 4) \text { or } \\
q \not \equiv 3 \epsilon(\bmod 4)\end{array}$ & 4 \\
\hline $\mathrm{P} \Omega_{n}^{+}(q)$ & $\mathcal{C}_{7}$ & $\mathrm{O}_{m}^{\epsilon}(q) \prec \mathrm{Sym}_{t}$ & $t>3 ;(\star)$ holds & 4 \\
\hline
\end{tabular}

As an illustrative example, consider the seventh row of Table 1 . Here, $G_{0}=$ $\mathrm{P} \Omega_{n}^{\epsilon}(q)$ and $H$ lies in class $\mathcal{C}_{1}$ of type $\mathrm{O}_{m}^{\epsilon_{1}}(q) \oplus \mathrm{O}_{n-m}^{\epsilon_{2}}(q)$, with $\epsilon, \epsilon_{1}, \epsilon_{2} \in$ $\{+,-\}$. Furthermore, $H$ is the stabilizer of a nondegenerate $m$-space $W$ of type $\epsilon_{1}$. 
In order for the group $H$ to need at least four generators, a number of conditions are necessary. First, $q$ must be odd, and $Q_{2}$ (the quadratic form induced by $H$ on $\left.W^{\perp}\right)$ has square discriminant. Also, $G$ must be contained in an $\operatorname{Aut}\left(G_{0}\right)$-conjugate of $\mathrm{P} \Gamma \mathrm{O}_{n}^{\epsilon}(q)$. Hence, we have that $G / G_{0}$ is a subgroup of $\mathrm{P} \Gamma \mathrm{O}_{n}^{\epsilon}(q)^{x} / \mathrm{P} \Omega_{n}^{\epsilon}(q)$, for some $x \in \operatorname{Aut}\left(G_{0}\right)$. Note that $\mathrm{P} \mathrm{O}_{n}^{\epsilon}(q)^{x} / \mathrm{P} \Omega_{n}^{\epsilon}(q) \cong K \times Z_{a f}$, where $(K, a)=$ $\left(D_{8}, 1\right)$, if $Q$ (the quadratic form induced by $G_{0}$ on $V$ ) has square discriminant, $(K, a)=\left(2^{2}, 1\right)$ if $Q$ has nonsquare discriminant and $\epsilon=+$ and $(K, a)=\left(Z_{2}\right.$, 2) if $Q$ has nonsquare discriminant and $\epsilon=-$ (see [31, Ch. 2]). Let $i$ denote the minimal number of generators for the intersection of $G / G_{0}$ with the normal factor $K$ of $\mathrm{P} \Gamma \mathrm{O}_{n}^{\epsilon}(q)^{x} / \mathrm{P} \Omega_{n}^{\epsilon}(q)$ (so that $i \in\{0,1,2\}$ ). Similarly, let $j$ denote the minimal number of generators for the intersection of $G / G_{0}$ with the Sylow 2-subgroup of the $Z_{a f}$ normal factor of $\mathrm{P} \Gamma \mathrm{O}_{n}^{\epsilon}(q)^{x} / \mathrm{P} \Omega_{n}^{\epsilon}(q)$ (so that $j \in\{0,1\}$ ). Then we must have $i+j \geqslant 2$. If all of these conditions are satisfied, then we have $d(H)=2+i+j$.

Part (ii) of the theorem shows that there exist infinitely many almost simple groups possessing a maximal subgroup requiring five generators. Thus, part (i) is best possible. We will also show that there are infinitely many pairs $(G, H)$ with the property that $G$ is a finite almost simple group; $H$ is a maximal subgroup of $G ; d(H)=3$; and $H$ does not have an elementary abelian factor group of order $2^{3}$. See Example 7.9 for an exposition of an infinite family of such examples. In particular, part (ii) is also best possible.

The following is an immediate consequence of Theorem 1.

COROLlARY 2. Let $G$ be a finite almost simple group with exceptional socle, and let $H$ be a maximal subgroup of $G$. Then $d(H) \leqslant 3$.

We also mention an application. In [11], a new equivalence relation $\equiv_{\mathrm{m}}$ among the elements of a finite group $G$ is defined, where two elements are equivalent if each can be substituted by the other in any generating set for $G$. This relation can be refined to a sequence $\equiv_{\mathrm{m}}^{(r)}$ of equivalence relations by saying that $x \equiv_{\mathrm{m}}^{(r)} y$ if each can be substituted by the other in any $r$-element generating set. The relations $\equiv_{\mathrm{m}}^{(r)}$ become finer as $r$ increases. The authors of [11] define a new group invariant $\psi(G)$ to be the value of $r$ at which they stabilize to $\equiv_{\mathrm{m}}$. They conjecture that $\psi(G) \in\{d(G), d(G)+1\}$ and use the main result in [10] to prove that $\psi(G) \leqslant 7$ for every almost simple group $G$ (see [11, Corollary 2.13]). Using Theorem 1, the better bound $\psi(G) \leqslant 6$ can be proved.

COROLlary 3. Let $G$ be a finite almost simple group. Then $\psi(G) \leqslant 6$.

The strategy for proving Theorem 1 can be described briefly as follows. By a result of Dalla Volta and the first author (see Proposition 2.6), $d(H)$ (for any 
finite group $H$ ) can be determined precisely from the non-Frattini chief factors of $H$ and the induced actions of $H$ on these factors (see Sections 2 and 3 for more details). To prove Theorem 1, we use detailed information on the structure of the maximal subgroups of finite almost simple groups to determine the chief factors of such subgroups and their associated actions. We remark that we only determine the action of $H$ on a chief factor $A$ of $H$ when it is necessary. For example, if we are trying to prove that the number of chief factors of size 3 in a class of groups is at most 2, then a group of shape $S . K$, where $S$ is a nonabelian simple group and $K$ has order 6 , clearly satisfies this property.

The layout of the paper is as follows: as mentioned above, our approach uses the theory of crowns in finite groups, which will be described in detail in Section 2. We will then conclude the section with a restatement of our theorem in this language (see Theorem 2.7). In Section 3, we prove a series of lemmas comprising the main tools which we will use to prove the theorem. We then subdivide our proof according to the classification of finite simple groups, beginning in Sections 4 and 5 with the proof of the theorem in the cases when $\operatorname{soc}(G)$ is a sporadic or an alternating group. We then move on to the classical cases not containing a so-called exceptional automorphism in Section 6, before completing the proof in Section 7, where we consider the almost simple groups with exceptional socle and the remaining classical cases (which only occur when $\left.\operatorname{soc}(G) \in\left\{\operatorname{Sp}_{4}\left(2^{f}\right), \mathrm{P} \Omega_{8}^{+}(q)\right\}\right)$. We conclude the paper with the above-mentioned examples of almost simple groups containing a maximal subgroup $H$ such that $d(H)=3$, but $H$ does not have an elementary abelian factor group of order $2^{3}$.

\section{Crowns in finite groups}

Let $G$ be a nontrivial finite group. In this section, we recall several notions in the theory of crowns in finite groups, including those of a chief series for $G$, a $G$-group, equivalent $G$-groups and monolithic primitive groups. We use these to express $d(G)$ as a function of the chief factors of $G$.

Recall that a chief series of a finite group $G$ is a normal series

$$
1=N_{0}<N_{1}<\cdots<N_{n}=G
$$

of finite length with the property that for $i \in\{0, \ldots, n-1\}, N_{i+1} / N_{i}$ is a minimal normal subgroup of $G / N_{i}$. The integer $n$ is called the length of the series and the factors $N_{i+1} / N_{i}$, where $0 \leqslant i \leqslant n-1$, are called the chief factors of the series. A nontrivial finite group $G$ always possesses a chief series. Moreover, two chief series of $G$ have the same length, and any two chief series of $G$ are the same up to permutation and isomorphism. Thus, adopting the notation above, we may define the chief length of $G$ to be $n$ and the chieffactors of $G$ to be the groups $N_{i+1} / N_{i}$. 
We can now begin our description of the theory of crowns in finite groups. The notion of a crown in a finite soluble group was defined by Gaschütz in [24], before being extended to all finite groups by Dalla Volta and the first author in [18]. A detailed exposition of the theory is also given in $[6,1.3]$.

We now require some terminology.

DEFINITION 2.1. A finite group $L$ is called monolithic if $L$ has a unique minimal normal subgroup $A$. If, in addition, $A$ is not contained in $\operatorname{Frat}(L)$, then $L$ is called a monolithic primitive group.

Let $L$ be a monolithic primitive group and let $A$ be its unique minimal normal subgroup. For each positive integer $k$, let $L^{k}$ be the $k$-fold direct product of $L$. The crown-based power of $L$ of size $k$ is the subgroup $L_{k}$ of $L^{k}$ defined by

$$
L_{k}=\left\{\left(l_{1}, \ldots, l_{k}\right) \in L^{k}: l_{1} \equiv \cdots \equiv l_{k} \bmod A\right\} .
$$

Equivalently, $L_{k}=A^{k} \operatorname{diag}\left(L^{k}\right)$, where $\operatorname{diag}\left(L^{k}\right):=\{(l, l, \ldots, l): l \in L\} \leqslant L^{k}$. We also define $L_{0}:=1$.

If a group $G$ acts on a group $A$ via automorphisms (that is, if there exists a homomorphism $G \rightarrow \operatorname{Aut}(A)$ ), then we say that $A$ is a $G$-group. If $G$ does not stabilize any nontrivial proper subgroup of $A$, then $A$ is called an irreducible $G$ group. Two $G$-groups $A$ and $B$ are said to be $G$-isomorphic, or $A \cong_{G} B$, if there exists a group isomorphism $\phi: A \rightarrow B$ such that $\phi(g(a))=g(\phi(a))$ for all $a \in A, g \in G$. Following [29], we say that two $G$-groups $A$ and $B$ are $G$ equivalent and we put $A \equiv_{G} B$, if there are isomorphisms $\phi: A \rightarrow B$ and $\Phi: A \rtimes G \rightarrow B \rtimes G$ such that the following diagram commutes:

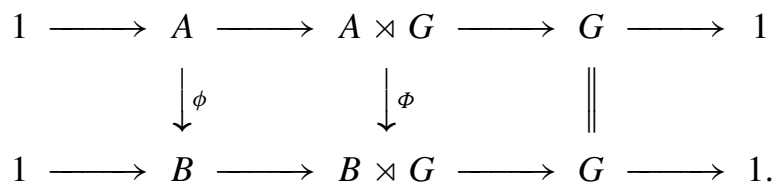

Note that two $G$-isomorphic $G$-groups are $G$-equivalent. In the particular case where $A$ and $B$ are abelian, the converse is true: if $A$ and $B$ are abelian and $G$-equivalent, then $A$ and $B$ are also $G$-isomorphic. It is proved (see, for example, [29, Proposition 1.4]) that two chief factors $A$ and $B$ of $G$ are $G$-equivalent if and only if either they are $G$-isomorphic or there exists a maximal subgroup $M$ of $G$ such that $G / \operatorname{core}_{G}(M)$ has two minimal normal subgroups $X$ and $Y$ that are $G$ isomorphic to $A$ and $B$, respectively. For example, the minimal normal subgroups of a crown-based power $L_{k}$ are all $L_{k}$-equivalent.

Recall that the Frattini group $\operatorname{Frat}(G)$ of a nontrivial finite group $G$ is nilpotent. The following terminology will be used frequently. 
DEFINITION 2.2. Let $G$ be a nontrivial finite group and let $H / K$ be a chief factor of $G$.

(i) We say that $H / K$ is Frattini if $H / K \leqslant \operatorname{Frat}(G / K)$.

(ii) We say that $H / K$ is complemented if there exists a subgroup $U$ of $G$ such that $U H=G$ and $U \cap H=K$. The group $U$ is said to be a complement of $H / K$ in $G$.

Recall that a subgroup $H$ of a group $G$ is said to supplement $K \leqslant G$ if $K H=G$. Since the Frattini subgroup of a finite group $G$ is nilpotent and the only subgroup supplementing $\operatorname{Frat}(G)$ is $G$ itself, the following lemma is immediate.

LEMMA 2.3. Let $G$ be a nontrivial finite group and let $A=H / K$ be a chief factor of $G$.

(i) If $A$ is abelian, then $A$ is non-Frattini if and only if $A$ is complemented.

(ii) If $A$ is nonabelian, then $A$ is non-Frattini.

For an irreducible $G$-group $A$, we define $\delta_{G}(A)$ to be the number of non-Frattini chief factors $G$-equivalent to $A$ in a chief series for $G$. Clearly, the number $\delta_{G}(A)$ does not depend on the choice of chief series for $G$. Denote by $L_{A}$ the monolithic primitive group associated with $A$. That is,

$$
L_{A}= \begin{cases}A \rtimes\left(G / C_{G}(A)\right) & \text { if } A \text { is abelian, } \\ G / C_{G}(A) & \text { otherwise. }\end{cases}
$$

If $A$ is a non-Frattini chief factor of $G$, then $L_{A}$ is a homomorphic image of $G$. More precisely, there exists a normal subgroup $N$ of $G$ such that $G / N \cong L_{A}$ and $\operatorname{soc}(G / N) \equiv_{G} A$. Consider now all the normal subgroups $N$ of $G$ with the property that $G / N \cong L_{A}$ and $\operatorname{soc}(G / N) \equiv_{G} A$ : the intersection $R_{G}(A)$ of all these subgroups has the property that $G / R_{G}(A)$ is isomorphic to the crown-based power $\left(L_{A}\right)_{\delta_{G}(A)}$. The socle $I_{G}(A) / R_{G}(A)$ of $G / R_{G}(A)$ is called the $A$-crown of $G$ and it is a direct product of $\delta_{G}(A)$ minimal normal subgroups $G$-equivalent to $A$.

Before proceeding, we briefly illustrate the definitions and terminology introduced above with an example. Let $V$ be a vector space of dimension $n$ over a field $\mathbb{F}$ of odd prime order $p$, and let $R \cong Z_{2}$ act on $V$ by inverting the nonzero elements of $V$. Set $G:=V \rtimes R$. Then $G$ has $n$ chief factors which are $G$-isomorphic to $A:=Z_{p}$. These chief factors are non-Frattini, noncentral and $G / C_{G}(A) \cong Z_{2}$. The group $G$ also has a central chief factor $B \cong Z_{2}$. Hence, we have $\delta_{H}(A)=n$ and $\delta_{H}(B)=1$. 
Proposition 2.4. Let $G$ be a nontrivial finite group and let $A=H / K$ be a non-Frattini chief factor of $G$. The following assertions hold:

(i) We have $I_{G}(A)=H C_{G}(A)$.

(ii) The group $I_{G}(A) / R_{G}(A)$ is the direct product of $\delta_{G}(A)$ non-Frattini chief factors of $G$ that are $G$-equivalent to A. Moreover, $\operatorname{soc}\left(G / R_{G}(A)\right)=$ $I_{G}(A) / R_{G}(A)$.

(iii) The group $G / R_{G}(A)$ is isomorphic to the crown-based power $\left(L_{A}\right)_{\delta_{G}(A)}$ of $L_{A}$ of size $\delta_{G}(A)$.

Proof. Part (i) is an easy exercise, while part (ii) follows from [23, Proposition 2.4]. Finally, part (iii) is [20, Proposition 9].

Next, we provide the key technical tools which will be used to prove Theorem 1 . The first reads as follows.

LEMMA 2.5. Let $G$ be a noncyclic finite group. The following assertions hold.

(i) There exist a monolithic primitive group $L$ and a positive integer $k$ such that $L_{k}$ is an image of $G$ and $d(G)=d\left(L_{k}\right)>d\left(L_{k-1}\right)$.

(ii) If $L$ is a monolithic primitive group such that $L_{k}$ is an image of $G$ for some positive integer $k$, then there exists a non-Frattini chief factor A of $G$ such that $L$ is isomorphic to the monolithic primitive group $L_{A}$ of $G$ associated with $A$ and $k \leqslant \delta_{G}(A)$. Moreover, if $d\left(L_{k}\right)=d(G)$, then $d\left(\left(L_{A}\right)_{\delta_{G}(A)}\right)=$ $d(G)$.

Proof. We consider part (i). Let $N$ be a normal subgroup of $G$ maximal with respect to $d(G / N)=d(G)$. Then, since $G$ is not cyclic, $d(H)<d(G)$ for any proper quotient $H$ of $G / N$. Set $K=G / N$. Note that $K$ is nontrivial and so $K$ has a minimal normal subgroup. Moreover, $K$ is not cyclic.

Suppose first that $K$ has a unique minimal normal subgroup $M$. Note that $M$ is a chief factor of $K$ and $\operatorname{soc}(K)=M$. By [37, Theorem 1.1], $d(K)=2$ and $K / M$ is cyclic. Since $d(K)=d(K / \operatorname{Frat}(K))$ and $d(H)<d(K)$ for any proper quotient $H$ of $K$, we must have $\operatorname{Frat}(K)=1$. In particular, $K$ is a monolithic primitive group with $d(K)>d(K / \operatorname{soc}(K))$. Suppose now that $K$ has two distinct minimal normal subgroups. The argument used in the proof of [18, Theorem 1.4] yields that there exist a monolithic primitive group $L$ and a positive integer $k$ such that $K \cong L_{k}$ and $d\left(L_{k}\right)>d\left(L_{k-1}\right)$. This proves part (i).

We now consider part (ii). By assumption, there exist a normal subgroup $N$ of $G$, a monolithic primitive group $L$ and a positive integer $k$ such that $G / N \cong L_{k}$. 
Let $B=\operatorname{soc}(L)$. Then $G$ has a non-Frattini chief factor $A$ isomorphic to $B$ and the monolithic primitive group $L_{A}$ of $G$ associated with $A$ is isomorphic to $L$. Note that a chief series for $L_{k}$ has $k$ non-Frattini chief factors $L_{k}$-equivalent to $B$. It follows that a chief series for $G$ has at least $k$ non-Frattini chief factors $G$-equivalent to $A$. In particular, $k \leqslant \delta_{G}(A)$. Suppose $d(G)=d\left(L_{k}\right)$. As $k \leqslant$ $\delta_{G}(A)$, we have $d\left(\left(L_{A}\right)_{\delta_{G}(A)}\right) \geqslant d(G)$. Proposition 2.4(iii) now yields $d(G)=$ $d\left(\left(L_{A}\right)_{\delta_{G}(A)}\right)$, as needed.

We now state and prove the main tool which will be used to prove our theorem.

Proposition 2.6. Let $G$ be a noncyclic finite group. For a non-Frattini chief factor $A$ of $G$, let $L_{A}$ be the monolithic primitive group of $G$ associated with $A$ and let $\delta_{G}(A)$ be the number of non-Frattini chief factors of $G$ in a chief series for $G$ which are $G$-equivalent to A. If $A$ is abelian, define

$$
\begin{aligned}
& r(A)=\operatorname{dim}_{\operatorname{End}_{L_{A} / A}(A)} A, \\
& s(A)=\operatorname{dim}_{E_{L_{A} / A}(A)} H^{1}\left(L_{A} / A, A\right), \\
& \theta(A)= \begin{cases}0 & \text { if } A \text { is central, } \\
1 & \text { otherwise, }\end{cases} \\
& h(A)=\theta(A)+\left\lceil\frac{\delta_{G}(A)+s(A)}{r(A)}\right\rceil .
\end{aligned}
$$

The following assertions hold:

(i) We have

$$
d(G)=\max _{A \text { non-Frattini }} d\left(\left(L_{A}\right)_{\delta_{G}(A)}\right),
$$

where the maximum is taken over all non-Frattini chieffactors $A$ of $G$.

(ii) Suppose that for every nonabelian chief factor $A=S^{n}$ of $G$, we have

$$
\delta_{G}(A) \leqslant \frac{|A|}{2 n|\operatorname{Out}(S)|} .
$$

Then either $d(G)=2$ or there is an abelian non-Frattini chief factor $B$ of $G$ such that $d(G)=d\left(\left(L_{B}\right)_{\delta_{G}(B)}\right) \geqslant 3$ and $d\left(L_{B}\right)>d\left(L_{B} / \operatorname{soc}\left(L_{B}\right)\right)$.

(iii) Let $\Phi$ be the set of abelian non-Frattini chief factors of $G$. Under the assumption of (ii), if $d(G)>2$, then

$$
d(G)=\max _{A \in \Phi} d\left(\left(L_{A}\right)_{\delta_{G}(A)}\right)=\max _{A \in \Phi} h(A) \leqslant \max _{A \in \Phi} \delta_{G}(A)+\theta(A) .
$$

Moreover, if $d(G)=d\left(\left(L_{A}\right)_{\delta_{G}(A)}\right)$, where $A \cong Z_{2}$ is a non-Frattini chief factor of $G$, then $d(G)=\delta_{G}(A)$. 
(iv) Under the assumption of (ii), if $\max _{A \in \Phi} h(A) \leqslant 3$, then $d(G) \leqslant 3$.

Proof. We first consider part (i). By Lemma 2.5, there exist a monolithic primitive group $L$ and a positive integer $k$ such that $L_{k}$ is an image of $G$ and $d(G)=$ $d\left(L_{k}\right)>d\left(L_{k-1}\right)$. Also there is a non-Frattini chief factor $A$ of $G$ isomorphic to $\operatorname{soc}(L)$ such that the monolithic primitive group $L_{A}$ associated with $G$ is isomorphic to $L$. Moreover, $d\left(\left(L_{A}\right)_{\delta_{G}(A)}\right)=d(G)$. In particular,

$$
d(G) \leqslant \max _{A \text { non-Frattini }} d\left(\left(L_{A}\right)_{\delta_{G}(A)}\right) .
$$

The result now follows from Proposition 2.4(iii). In the remaining part of the proof, we will implicitly use the following consequence of part (i): there is a non-Frattini chief factor $A$ of $G$ such that $d(G)=d\left(\left(L_{A}\right)_{\delta_{G}(A)}\right)$ and $d\left(L_{A}\right)>$ $d\left(L_{A} / \operatorname{soc}\left(L_{A}\right)\right)$.

We now consider part (ii). By Lemma 2.5, there is a non-Frattini chief factor $C$ of $G$ such that $d(G)=d\left(\left(L_{C}\right)_{\delta_{G}(C)}\right)>d\left(L_{C} / \operatorname{soc}\left(L_{C}\right)\right)$. Suppose, for a contradiction, that $d(G)>2$ and there is no non-Frattini abelian chief factor $B$ of $G$ such that $d(G)=d\left(\left(L_{B}\right)_{\delta_{G}(B)}\right) \geqslant 3$ and $d\left(L_{B}\right)>d\left(L_{B} / \operatorname{soc}\left(L_{B}\right)\right)$. Then by part (i), $C$ must be nonabelian. Write $C=S^{n}$, where $S$ is a nonabelian simple group, set $L=L_{C}$ and recall that $\operatorname{soc}(L) \cong C$. Since $L$ is a monolithic primitive group with nonabelian socle, by [19, Corollary 8], there is a function $\psi_{L}: \mathbb{N} \rightarrow \mathbb{N}$ such that for every $s \geqslant d(L)$, we have

$$
k \leqslant \psi_{L}(s) \text { if and only if } d\left(L_{k}\right) \leqslant s .
$$

Moreover, by [19, Proposition 10], there is an absolute constant $\gamma$ such that if $s \geqslant d(L)$, then

$$
\psi_{L}(s) \geqslant \frac{\gamma|C|^{s-1}}{n|\operatorname{Out}(S)|}
$$

and by [38, Corollary 1.2] and [21, Corollary 1.2], $\gamma \geqslant 1 / 2$. We claim that $d(L) \leqslant$ 2. Suppose not. Since $\delta_{G}(C) \leqslant|C| /(2 n|\operatorname{Out}(S)|)$, the bounds in (2.1) and (2.2) imply that $d\left(L_{\delta_{G}(C)}\right) \leqslant d(L)$ and so $d(L)=d\left(L_{\delta_{G}(C)}\right)$. In particular, $d(L)>$ $d(L / \operatorname{soc}(L))$. Since $L$ is a monolithic primitive group and $L$ is not cyclic, by [37, Theorem 1.1], $d(L)=\max (2, d(L / \operatorname{soc}(L)))$. It follows that $d(L)=2$, a contradiction. Arguing by contradiction, we have established the claim, namely $d(L) \leqslant 2$. Since $d(L) \leqslant 2$ and $\delta_{G}(C) \leqslant|C| /(2 n|\operatorname{Out}(S)|)$, setting $s=2$ in (2.1) and (2.2), we obtain $d\left(L_{\delta_{G}(C)}\right) \leqslant 2$ and so $d(G) \leqslant 2$, a contradiction. This final contradiction establishes part (ii).

We now consider part (iii). Since $d(G)>2$, by part (ii), there is an abelian non-Frattini chief factor $B$ of $G$ such that $d(G)=d\left(\left(L_{B}\right)_{\delta_{G}(B)}\right)$ and $d\left(L_{B}\right)>$ $d\left(L_{B} / \operatorname{soc}\left(L_{B}\right)\right)$. Since $d\left(L_{B}\right)>d\left(L_{B} / \operatorname{soc}\left(L_{B}\right)\right)$, [19, Proposition 6] gives 
$d\left(\left(L_{B}\right)_{\delta_{G}(B)}\right)=h(B)$. By [2, Theorem A] $s(B)<r(B)$ and so $h(B) \leqslant \theta(B)+$ $\delta_{G}(B)$. Finally, if $d(G)=d\left(\left(L_{A}\right)_{\delta_{G}(A)}\right)$, where $A \cong Z_{2}$ is a non-Frattini chief factor of $G$, then $\left(L_{A}\right)_{\delta_{G}(A)} \cong Z_{2}^{\delta_{G}(A)}$ and so $d(G)=\delta_{G}(A)$. Part (iii) follows.

Finally, part (iv) follows immediately from parts (i), (ii) and (iii).

We can now restate our main theorem in the language of crowns. Note that a finite group $H$ always has a non-Frattini chief factor. Indeed, a minimal normal subgroup of $H / \operatorname{Frat}(H)$ is always a non-Frattini chief factor of $H$.

THEOREM 2.7. Let $G$ be a finite almost simple group with socle $G_{0}$. Fix a maximal subgroup $H$ of $G$ and a non-Frattini chieffactor $A$ of $H$.

(i) If $A$ is nonabelian, then $\delta_{H}(A) \leqslant 2$.

(ii) If $A$ is abelian but noncentral, then $\delta_{H}(A) \leqslant 2$.

(iii) If $A$ is central, then $\delta_{H}(A) \leqslant 3$, unless $|A|=2$ and $(G, H)$ is one of the pairs described in part (ii) of Theorem 1. In this latter case, $\delta_{H}(A)=4$ if $G$ is as in part (ii)(a) of Theorem 1. If $G$ is as in part $(i i)(b)$, then $\delta_{H}(A)=d$, where $d$ is as in the last column of Table 1.

We now show that, in fact, Theorem 2.7 implies Theorem 1. Indeed, suppose that Theorem 2.7 holds, and let $H$ and $G$ be as in the statement of Theorem 1 . By [42, Lemma 2.1], we have $|\operatorname{Out}(S)| \leqslant \log |S|$ for every nonabelian finite simple group $S$. Hence, the bound $|S|^{n} /(2 n|\operatorname{Out}(S)|) \geqslant 2$ holds. It then follows from Proposition 2.6 that $d(H) \leqslant \max _{A}\left(\delta_{H}(A)+\theta(A)\right)$ as $A$ runs over the abelian non-Frattini chief factors of $H$. Theorem 1 then follows immediately from Theorem 2.7 and the definition of $\theta$.

\section{Chief factors of finite groups}

As shown in the last section, to prove Theorem 1, it will suffice to prove Theorem 2.7. This will be our aim for the remainder of the paper. In this section, we prove some technical lemmas which will be crucial in our proof of Theorem 2.7. The first three are elementary but will be used frequently. Before we state them, we require a definition.

DeFinition 3.1. Let $G$ be a finite group.

(a) A subsection of $G$ is a group $N / M$, where $N \leqslant G$ and $M$ is normal in $N$. A subsection $N / M$ is called a section of $G$ if $N$ and $M$ are both normal in $G$. 
(b) Let $N / M$ be a section of $G$, and let $A$ be a non-Frattini chief factor of $G$. Then $N R_{G}(A) / M R_{G}(A)$ is a normal subgroup of $G / R_{G}(A)$, so the intersection of $N R_{G}(A) / M R_{G}(A)$ with the socle $\operatorname{soc}\left(G / R_{G}(A)\right)$ is isomorphic to $A^{m}$, for some $m \geqslant 0$. We define $\delta_{G, N / M}(A):=m$. That is, $\delta_{G, N / M}(A)$ is the number of non-Frattini chief factors of $G$ which are $G$ equivalent to $A$ and appear as a section of $N / M$.

REMARK 3.2. Let $G$ be a finite group, and let $N / M$ be a section of $G$. If $\delta_{G, N / M}(A)>0$ for some chief factor $A$ of $G$, that is, if $A$ appears as a section of $N / M$, then we will write $A \preccurlyeq N$.

We begin the series of lemmas mentioned above with a result concerning chief factors in groups with a cyclic normal subgroup: its proof is an easy consequence of the Jordan-Hölder theorem.

LEMMA 3.3. Let $G$ be a finite group having a cyclic normal subgroup $N$, say $N=Z_{a}$ for some $a \in \mathbb{N}$, and set $J=G / N$. The following assertions hold:

(i) A chieffactor of $G$ is either a section of $N$ or $J$.

(ii) If $J$ has a cyclic normal subgroup $M / N$, say $M / N=Z_{b}$ for some $b \in \mathbb{N}$, then a chief factor of $G$ is either a section of $N, M / N$ or $G / M$.

The next result is a useful reduction lemma.

LEMMA 3.4. Let $G$ be a finite group, and let $A$ be a non-Frattini chieffactor of G. Suppose that $1=N_{0} \leqslant N_{1} \leqslant \cdots \leqslant N_{k}=G$ is a normal series for $G$. Then

(i) $\delta_{G}(A)=\sum_{i=1}^{k} \delta_{G, N_{i} / N_{i-1}}(A)$.

(ii) $\delta_{G, G / N_{i}}(A)=\delta_{G / N_{i}}(A)$.

(iii) If $N_{i} / N_{i-1}$ is cyclic, then $\delta_{G, N_{i} / N_{i-1}}(A) \leqslant 1$.

Proof. If $N$ is normal in $G$ and $N R_{G}(A) / R_{G}(A) \cong A^{m}$, then the group $\operatorname{soc}\left(G / R_{G}(A)\right)$ modulo $N R_{G}(A) / R_{G}(A)$ is clearly isomorphic to $A^{\delta_{G}(A)-m}$. Hence, $\delta_{G}(A)=\delta_{G, N}(A)+\delta_{G, G / N}(A)$. Part (i) now follows by an easy inductive argument.

Since $\operatorname{Frat}\left(G / N_{i}\right)=\operatorname{Frat}(G) N_{i} / N_{i}$, part (ii) follows, and since the non-Frattini chief factors of a cyclic group are precisely the (cyclic) prime factors of its unique square-free quotient, part (iii) follows. 
Lemma 3.5. Let $G$ be a finite perfect group. Then $Z(G) \leqslant \operatorname{Frat}(G)$. In particular, this holds if $G$ is quasisimple.

Proof. Suppose that $z$ is an element of $Z(G)$ of prime order and that $z$ is not in $\operatorname{Frat}(G)$. Then there exists a maximal subgroup $H<G$, with $z \notin H$. Hence, $G=H\langle z\rangle$. It then follows that $H \unlhd G$. This contradicts $G$ being perfect since $G / H$ is abelian in this case. The result follows.

We now investigate the case where certain subgroups of wreath products appear as sections in finite groups. This will be especially useful in our work on the $\mathcal{C}_{2}$ and $\mathcal{C}_{7}$ families in the classical cases. First, we need two definitions.

DEFINITION 3.6. Let $Q$ be a finite abelian group. The subgroup

$$
K:=\left\{\left(x_{1}, \ldots, x_{t}\right): \prod_{i} x_{i}=1\right\}
$$

of $Q^{t}$ is called the deleted subgroup of $Q^{t}$.

REMARK 3.7. If the group $Q$ in Definition 3.6 is elementary abelian of order $p^{a}$ for a prime $p$, then $K$ is a module for the group $J:=\mathrm{Sym}_{t}$, via permutation of coordinates. It is called the deleted permutation module for $J$ and it has dimension $a(t-1)$ over the field $\mathbb{F}_{p}$. In particular, when $Q=\mathbb{F}_{p}, K$ and $W=\{(f, \ldots$, $\left.f) \mid f \in \mathbb{F}_{p}\right\}$ are the unique nontrivial proper $J$-submodules of $\mathbb{F}_{p}^{t}$ and $K / K \cap W$ is called the fully deleted permutation module for $J$.

Lemma 3.9 requires a careful analysis of the chief factors of certain subgroups in a wreath product $E ₹ J$, where $E$ is a finite group, and $J:=\operatorname{Sym}_{t}$. We will denote the base group of such a wreath product by $B=B(E \geq J)$. We will view $B$ as the direct product $B=E_{1} \times \cdots \times E_{t}$ of $t$ copies of $E$, and for a subgroup $L$ of $E$, we will write $L_{i}$ for the corresponding subgroup of $E_{i}$. Furthermore, we will write $B_{L}:=L_{1} \times \cdots \times L_{t}$. We will frequently use, and make no further mention, of these conventions.

Before stating Lemma 3.9, we need to introduce some additional terminology.

DEFINITION 3.8. Let $E$ be a finite group, let $t \geqslant 2$ be a positive integer, and consider the wreath product $E \imath J$, where $J:=\operatorname{Sym}_{t}$. Let $B=B(E \imath J)$ be the base group. We call a subgroup $H$ of $E \geq J$ extra large if all of the following conditions are satisfied:

(a) $H \cap J \in\left\{\mathrm{Alt}_{t}, \mathrm{Sym}_{t}\right\}$. 
(b) $H \cap B$ contains $B_{F}$ for some normal subgroup $F$ of $E$ such that

(i) $E / F$ has abelian Frattini quotient $E / \operatorname{Frat}(E) F$;

(ii) $\operatorname{Frat}(E) \cap F=\operatorname{Frat}(F)$;

(iii) $\delta_{E, F}(W) \leqslant 1$ for all non-Frattini chief factors $W$ of $E$.

(c) $(H \cap B) /\left(H \cap B_{\text {Frat }(E) F}\right)$ is the deleted subgroup of $(E / \operatorname{Frat}(E) F)^{t}$.

The normal subgroup $F$ of $E$ above will be called a source of $H$.

LEMMA 3.9. Let $H$ be an extra large subgroup of a wreath product $E ? J$, where $E$ is a finite group and $J:=\operatorname{Sym}_{t}$, with $t \geqslant 2$. Let $B=B(E<J)$ be the base group, and let $F \unlhd E$ be a source of $H$. Assume that $G$ is a finite group with a normal series

$$
1<H \leqslant G
$$

such that $N_{G}\left(F_{1}\right) H=G$. Let $W$ be a non-Frattini chief factor of $E$.

(i) Suppose that $W \preccurlyeq F$ is noncentral and that if $t=2$, then $E / C_{E}(W)$ is not an elementary abelian 2-group. Then $B_{W} \cong W^{t}$ is a noncentral chief factor of $G$ contained in $H \cap B$.

(ii) Suppose that either $W \preccurlyeq F$ is central or that $t=2$ and $E / C_{E}(W)$ is an elementary abelian 2-group. Let $A_{\text {diag }}$ (respectively $A_{\text {full }}$ ) be the diagonal (respectively fully deleted) permutation modules for $J$ over the field $\mathbb{F}_{p}$, where $p=|W|$.

(a) Assume that $p \mid t$. Then $A_{\text {diag }}, A_{\text {full }} / A_{\text {diag }}$ and $B_{W} / A_{\text {full }}$ are chieffactors of $G$ contained in $H \cap B$. Furthermore, $A_{\text {diag }}$ is Frattini since it is not complemented in $B_{W}$.

(b) Assume that $p \nmid t$. Then the $G$-module $B_{W} \cong W^{t}$ splits into a direct sum of two $G$-chief factors: the diagonal subgroup $A_{\text {diag }}$ of $B_{W}$ and the fully deleted permutation module $A_{\text {full }}$ in $B_{W}$.

(iii) Suppose that $W \preccurlyeq E / F$.

(a) Assume that $p \mid t$. Then $A_{\text {diag }}$ and $A_{\text {full }} / A_{\text {diag }}$ are chief factors of $G$ contained in $H \cap B$. Furthermore, $A_{\text {diag }}$ is Frattini since it is not complemented in $A_{\text {full. }}$.

(b) Assume that $p \nmid t$. Then the fully deleted permutation module $A_{\text {full }}$ is a chief factor of $G$ contained in $H \cap B$. In particular, we get one central $G$-chieffactor and one noncentral $G$-chieffactor of order $|W|^{t-1}$. 
Finally, the group $H \cap B$ is normal in $G$, and the non-Frattini $G$-chief factors contained in $H \cap B$ are a subset of the groups listed in (i), (ii) and (iii) above.

Proof. We prove the claim by induction on $|E|$. If $E=1$, then our statement is trivially true, so we may assume $E \neq 1$. Identify $E$ with $E_{1}$, and for any subgroup $K$ of $E$, recall that we write $K_{i}$ for the corresponding subgroup of $E_{i}$ and $B_{K}$ for the group $K_{1} \times K_{2} \times \cdots \times K_{t} \leqslant B$. Let $\pi_{i}: B \rightarrow E_{i}$ be the projection maps. First, note that the condition $N_{G}\left(F_{1}\right) H=G$ implies that $H$ acts transitively on the set $\Sigma$ of $G$-conjugates of $F_{1}$. Since $H \cap B$ is precisely the kernel of the action of $H$ on $\Sigma$, and $H \unlhd G$, it follows that $H \cap B \unlhd G$, as claimed. Note also that $H / H \cap B$ is isomorphic to either Alt $_{t}$ or $\operatorname{Sym}_{t}$.

We now examine the chief factors of $G$ contained in $H \cap B$. Suppose first that $F$ is nontrivial, and let $W$ be a minimal normal subgroup of $E$ contained in $F$. The group $N_{G}\left(F_{1}\right)$ acts on $F_{1}$ via automorphisms, and the associated $N_{G}\left(F_{1}\right)$ conjugates of $W$ are normal subgroups of $F$ contained in $\operatorname{soc}(F)$. Let $X$ be the product of the distinct $N_{G}\left(F_{1}\right)$-conjugates of $W$ in $E$. Then $X$, being (equivalent to) a normal subgroup of $N_{G}\left(F_{1}\right) \geqslant E_{1}$, is a normal subgroup of $E$. Hence, the condition $N_{G}\left(F_{1}\right) H=G$ implies that $B_{X}$ is normal in $G$.

Suppose first that $W \leqslant \operatorname{Frat}(E)$. Then $W \leqslant \operatorname{Frat}(E) \cap F=\operatorname{Frat}(F)$. Moreover, $\operatorname{Frat}\left(F_{1}\right)$ char $F_{1}$, so $N_{G}\left(F_{1}\right)$ normalizes Frat $\left(F_{1}\right)$. Hence, $X \leqslant \operatorname{Frat}(F)$ as well. Thus, $B_{X} \leqslant \operatorname{Frat}(F)^{t} \leqslant \operatorname{Frat}\left(B_{F}\right)$. Since $B_{F}$ is subnormal in $G$, it follows that $B_{X} \leqslant \operatorname{Frat}(G)$. Now, $H / B_{X} \leqslant(E / X) 2 J$, and the series $1<H / B_{X} \leqslant G / B_{X}$ satisfies the hypothesis of the lemma, with $E$ replaced by $E / X$ and $F$ replaced by $F / X$. The result then follows from the inductive hypothesis.

So we may assume that $W$ is not contained in the Frattini subgroup of $E$. Then $X \cong W^{m}$, where $0 \leqslant m \leqslant \delta_{E, F}(W)=1$. Hence, we must have $X=W$ since $X$ is nontrivial. In particular, it follows that $W_{1}$ is normalized by $N_{G}\left(F_{1}\right)$ and that $B_{W}=B_{X}$ is normal in $G$. We wish to examine the $G$-chief factors contained in $B_{W}$. We distinguish two cases:

(1) $W$ is nonabelian. Then $W \cong T^{a}$ for some nonabelian simple group $T$ and some positive integer $a$. We claim that $B_{W}$ is, in fact, a minimal normal subgroup of $G$ in this case. To prove this, suppose that $K$ is any nontrivial normal subgroup of $G$ contained in $B_{W}$. Then $K^{\pi_{i}} \unlhd(H \cap B)^{\pi_{i}}=E_{i}$, so $K^{\pi_{i}}$ is a normal subgroup of $E_{i}$ contained in $W_{i}$. Since $K$ is nontrivial and $H \cap J$ is transitive, it follows that $K^{\pi_{i}}=W_{i}$ for all $i$. Thus, $K \leqslant B_{W}$ is a subdirect product of $T^{a t}$. Since $K \unlhd B_{W}$ and $T$ is a nonabelian simple group, it follows that $K=B_{W}$, so $B_{W}$ is a minimal normal subgroup of $G$.

(2) $W$ is abelian. Then $|W|=p^{a}$, for some prime $p$. For ease of notation, set $Y:=B_{W}$. Let $D \cong F$ be the diagonal subgroup of $B_{F}$, and consider 
the subgroup $I:=D \times(H \cap J) \leqslant(H \cap B)(H \cap J) \leqslant H$. By [31, Proposition 5.3.4], the $(H \cap J)$-module $Y \downarrow_{H \cap J}$ has an $(H \cap J)$-series

$$
1=Y_{0}<Y_{1}<Y_{1}+Y_{2} \leqslant Y_{3}=Y,
$$

where $Y_{1}$ is the diagonal subgroup of $Y$ and splits into a direct sum of $a$ copies of the trivial $\mathbb{F}_{p}[H \cap J]$-module; $Y_{2}$ splits into a direct sum of $a$ copies of the (irreducible) fully deleted permutation module for $H \cap J$ over $\mathbb{F}_{p}$. Also, $Y=Y_{3}=Y_{1}+Y_{2}$ if $p \nmid t$. If $p \mid t$, then $Y /\left(Y_{1}+Y_{2}\right)$ is a direct sum of $a$ copies of the trivial $\mathbb{F}_{p}[H \cap J]$-module. Next, $Y \downarrow_{D}$ splits as a direct sum

$$
Y=W_{1} \oplus W_{2} \oplus \cdots \oplus W_{t} .
$$

(We caution the reader that although the $W_{i}$ are completely reducible by Clifford's theorem (see [16, Theorem 49.2]), they are not necessarily irreducible as $D$-modules.) It follows that $Y \downarrow_{I}$ has an $I$-series

$$
1=A_{0}<A_{1} \leqslant A_{1}+A_{2} \leqslant A_{3}=Y,
$$

where $A_{1} \cong W_{1} \otimes 1$ has dimension $a$ and $A_{2} \cong Y_{2}$ has dimension $a(t-1)$. Furthermore, if $p \nmid t$, then $A_{1} \cap A_{2}$ is trivial and $Y=A_{1} \oplus A_{2}$. If $p \mid t$, then $A_{1} \leqslant A_{2}$ and $Y / A_{2}$ has dimension $a$.

Now, if $W$ is central, then $a=1$ and, as an $H$-series, the series in (3.2) is equivalent to the series in (3.1). Hence, it is, in fact, an irreducible $\mathrm{H}$ series. This is because $H \cap B$ is in the kernel of the action of $H$ on $Y$ in this case, so we can just view $Y$ as a module for $H / H \cap B$, which is isomorphic to either Alt $_{t}$ or $\mathrm{Sym}_{t}$. The irreducibility of the factors then follows from [31, Proposition 5.3.4], as above. Since $W_{1}$ and $B_{W_{1}}$ are both normalized by $N_{G}\left(F_{1}\right)$, the series (3.2) is also fixed by $N_{G}\left(F_{1}\right)$. Hence, (3.2) is a $G$-series with irreducible factors since $G=H N_{G}\left(F_{1}\right)$.

So we may assume that $W$ is noncentral. If $W_{1}$ is not in $Z\left(F_{1}\right)$, then we may choose an element $x \in B_{F}$ with the property that $x^{\pi_{1}}$ does not centralize $A_{1}$, and $x^{\pi_{i}}=1$ for $i \geqslant 2$. If $W_{1} \leqslant Z\left(F_{1}\right)$ and $t>2$, then choose $e \in E$ such that $e$ does not centralize $W$. Then we may choose an element $x \in H \cap B$ with $x^{\pi_{1}} \in F_{1} e, x^{\pi_{2}} \in F_{2} e^{-1}$ and $x^{\pi_{i}} \in F_{i}$ for $i>2$. If $W_{1} \leqslant Z\left(F_{1}\right), t=2$, and there exists $e \in E$ such that $e^{2}$ acts nontrivially on $W$, then we may choose an element $x \in H \cap B$ with $x^{\pi_{1}} \in F_{1} e$ and $x^{\pi_{2}} \in F_{2} e^{-1}$. Then in each case, neither $A_{1}$ nor $A_{2}$ are $\langle x\rangle$-modules, so neither of them are $(H \cap B)$ submodules of $Y$. Thus, (3.2) implies that in both cases, $Y$ must be irreducible as an $(H \cap B)(H \cap J)$-module and, hence, irreducible as a $G$-module.

Finally, if $W_{1} \leqslant Z\left(F_{1}\right), t=2$, and $E / C_{E}(W)$ is an elementary abelian 2group, then arguing as in the central case above, it is easy to see that (3.2) is, in fact, a $G$-series with irreducible factors. 
Finally, we apply our inductive hypothesis: we have a normal series

$$
1<H / B_{W} \leqslant G / B_{W},
$$

where $H / B_{W} \leqslant(E / W)$ 乙 $J$, and

$$
N_{G / B_{W}}\left(F_{1} / W_{1}\right) H / B_{W} \geqslant\left(N_{G}\left(F_{1}\right) B_{W} / B_{W}\right) \cdot\left(H / B_{W}\right)=G / B_{W} .
$$

The assumptions of the lemma are now satisfied, with $E$ replaced by $E / W$ and $F$ replaced by $F / W$.

This gives us the chief factors of $G$ contained in $B_{F}$. To find the chief factors of $G$ contained in $H \cap B / B_{F}$, note that $H \cap B / B_{F}$ is the fully deleted subgroup of $(E / F)^{t}$. Let $W$ be a non-Frattini chief factor of $E / F$. Then $W$ is central since $E / F$ is abelian. Let $A_{\text {diag }}$ (respectively $A_{\text {full }}$ ) be the diagonal (respectively fully deleted) permutation modules. Then, arguing as in the central case above, we see that

(a) If $p \mid t$, then $A_{\text {diag }}$ and $A_{\text {full }} / A_{\text {diag }}$ are the chief factors of $G$ contained in $H \cap B / B_{F}$. Furthermore, $A_{\text {diag }}$ is Frattini since it is not complemented in $A_{\text {full. }}$.

(b) If $p \nmid t$, then the fully deleted permutation module $A_{\text {full }}$ is a chief factor of $G$ contained in $H \cap B$.

Hence, the chief factors of $G$ contained in $H \cap B$ are a subset of those stated in parts (i)-(iii) of the lemma.

The following will allow us to apply Lemma 3.9 in our proof of Theorem 2.7 in the classical case.

LEMMA 3.10. Let $E:=\mathrm{PX}_{m}(q)$ and $F:=\mathrm{PY}_{m}(q)$, where $(\mathrm{X}, \mathrm{Y})$ runs through the symbols $\left\{(\mathrm{GL}, \mathrm{SL}),(\mathrm{GU}, \mathrm{SU}),(\mathrm{GSp}, \mathrm{Sp}),\left(\mathrm{GO}^{\epsilon}, \Omega^{\epsilon}\right)\right\}$. Then

(i) $F \geqslant E^{\prime}$;

(ii) $\operatorname{Frat}(E) \cap F=\operatorname{Frat}(F)$;

(iii) all chief factors of $E$ contained in $F$ are noncentral;

(iv) $\delta_{E, F}(W) \leqslant 1$ for all non-Frattini chieffactors $W$ of $E$.

Proof. In most cases, the group $E$ is almost simple with socle $F$ and $E / F$ is abelian. In these cases, therefore, the result is clear. If $m=1$, then $F=1$, and again the result is clear. The other possibilities for $\mathrm{PY}_{m}(q)$ are listed in [31, Proposition 2.9.2], and the result can be checked by direct computation in these cases. 
We conclude this section by recording some important results concerning the outer automorphism groups of the nonabelian finite simple groups. Recall that a finite simple group $G_{0}$ of Lie type occurs as the derived subgroup of the fixed point group of a simple algebraic group $\mathbf{G}$ of adjoint type, defined over an algebraically closed field of prime characteristic $p$, under a Steinberg endomorphism $\sigma$, that is, $G_{0}=\left(\mathbf{G}_{\sigma}\right)^{\prime}$. We use the standard notation $G_{0}=\left(\mathbf{G}_{\sigma}\right)^{\prime}=$ $\mathbf{G}(q)$, where $q=p^{f}$ for some positive integer $f$. (We include the possibility that $\mathbf{G}(q)$ is of twisted type.) Also if $G_{0}$ is of orthogonal type with associated nondegenerate quadratic form $Q$ and $q$ is odd, we let $D(Q)$ be the discriminant of $Q$ (see [31, Section 2.5]).

Proposition 3.11 [15, Table 5]. Let $G_{0}$ be a finite simple group. The outer automorphism group $\operatorname{Out}\left(G_{0}\right)$ of $G_{0}$ is given in Table 2.

The next result follows from [17]. We include a different proof illustrating the method of crowns.

Proposition 3.12. Let $G$ be a finite almost simple group and let $G_{0}=\operatorname{soc}(G)$. Then $d(G) \in\{2,3\}$. Moreover, $d(G)=3$ if and only if $G$ has a central nonFrattini chief factor $A \cong Z_{2}$ with $\delta_{G}(A)=3$. In particular, if $d(G)=3$, then either

(i) $G_{0}=\mathrm{L}_{n}(q)$, where $n \geqslant 4$ is even and $q=p^{f}$ is odd with $f$ even or

(ii) $G_{0}=\mathrm{P} \Omega_{n}^{ \pm}(q)$, where $q=p^{f}$ is odd with $f$ even.

Proof. Since $G_{0}$ is the only nonabelian chief factor of $G$, we have $\delta_{G}\left(G_{0}\right)=1$. In particular, $2 \delta_{G}\left(G_{0}\right)\left|\operatorname{Out}\left(G_{0}\right)\right|<\left|G_{0}\right|$, and so Proposition 2.6 implies that $d(G)=$ 2 or

$$
\begin{aligned}
d(G) & =\max _{A \in \Phi} d\left(L_{A, \delta_{G}(A)}\right)=\max _{A \in \Phi} \theta(A)+\left\lceil\frac{\delta_{G}(A)+s(A)}{r(A)}\right\rceil \\
& \leqslant \max _{A \in \Phi} \theta(A)+\delta_{G}(A),
\end{aligned}
$$

where $\Phi, \theta, r$ and $s$ are defined in Proposition 2.6. Suppose that $d(G)>2$. Let $A$ be a non-Frattini abelian chief factor of $G$. Then $A$ can be viewed as a chief factor of $G / G_{0} \leqslant \operatorname{Out}\left(G_{0}\right)$. Set $K=G / G_{0}$.

Without loss of generality, suppose $d(G)=d\left(L_{A, \delta_{G}(A)}\right)$. Note that $\operatorname{Out}\left(G_{0}\right)$ is not cyclic as otherwise $\delta_{G}(A)=1$, contradicting $d(G)>2$.

We claim that $\operatorname{Out}\left(G_{0}\right)$ is not the semidirect product of two nontrivial cyclic groups. Suppose otherwise. Say $\operatorname{Out}\left(G_{0}\right)=N H$ where $N \triangleleft \operatorname{Out}\left(G_{0}\right), H \cong$ 
Table 2. The outer automorphism group of a finite simple group.

\begin{tabular}{|c|c|}
\hline$G_{0}$ & $\operatorname{Out}\left(G_{0}\right)$ \\
\hline $\mathrm{Alt}_{n}, n \geqslant 5$ & $\begin{cases}Z_{2} & \text { if } n \neq 6 \\
Z_{2} \times Z_{2} & \text { if } n=6\end{cases}$ \\
\hline $\mathrm{L}_{n}(q), n \geqslant 2$ & $\begin{cases}\left(Z_{(n, q-1)}: Z_{f}\right): Z_{2} & \text { if } n \geqslant 3 \\
Z_{(2, q-1)} \times Z_{f} & \text { if } n=2\end{cases}$ \\
\hline $\mathrm{U}_{n}(q), n \geqslant 3$ & $\begin{array}{l}Z_{(n, q+1)}: Z_{2 f} \\
Z_{2} \times Z_{f} \text { if } q \text { odd }\end{array}$ \\
\hline $\operatorname{PSp}_{2 m}(q), m \geqslant 2$ & $\begin{cases}Z_{f} & \text { if } m \geqslant 3 \text { and } q \text { even } \\
Z_{2 f} & \text { if } m=2 \text { and } q \text { even }\end{cases}$ \\
\hline$\Omega_{2 m+1}(q), m \geqslant 3$ and $q$ odd & $Z_{2} \times Z_{f}$ \\
\hline $\mathrm{P} \Omega_{8}^{+}(q)$ & $\begin{cases}\operatorname{Sym}_{4} \times Z_{f} & \text { if } q \text { odd } \\
\operatorname{Sym}_{3} \times Z_{f} & \text { if } q \text { even }\end{cases}$ \\
\hline $\mathrm{P} \Omega_{2 m}^{+}(q), m>4$ & $\begin{cases}D_{8} \times Z_{f} & \text { if } q \text { odd and } D(Q) \text { square } \\
Z_{2} \times Z_{2} \times Z_{f} & \text { if } q \text { odd and } D(Q) \text { nonsquare } \\
Z_{2} \times Z_{f} & \text { if } q \text { even }\end{cases}$ \\
\hline $\mathrm{P} \Omega_{2 m}^{-}(q), m \geqslant 4$ & $\begin{array}{ll}Z_{2} \times Z_{2 f} & \text { if } q \text { odd and } D(Q) \text { nonsquare } \\
Z_{2 f} & \text { if } q \text { even }\end{array}$ \\
\hline$G_{2}(q)$ & $\begin{array}{ll}Z_{f} & \text { if } p \neq 3 \\
Z_{f}: Z_{2} & \text { if } p=3\end{array}$ \\
\hline$F_{4}(q)$ & $\begin{array}{ll}Z_{f} & \text { if } p \neq 2 \\
Z_{f}: Z_{2} & \text { if } p=2\end{array}$ \\
\hline$E_{6}(q)$ & $\left(Z_{(3, q-1)}: Z_{f}\right): Z_{2}$ \\
\hline$E_{7}(q)$ & $Z_{(2, q-1)} \times Z_{f}$ \\
\hline$E_{8}(q)$ & $Z_{f}$ \\
\hline${ }^{2} B_{2}(q), q=2^{2 m+1}, m \geqslant 1$ & $Z_{f}$ \\
\hline${ }^{2} G_{2}(q), q=3^{2 m+1}, m \geqslant 1$ & $Z_{f}$ \\
\hline${ }^{2} F_{4}(q), q=2^{2 m+1}, m \geqslant 1$ & $Z_{f}$ \\
\hline${ }^{2} F_{4}(2)^{\prime}$ & $Z_{2}$ \\
\hline${ }^{3} D_{4}(q)$ & $Z_{3 f}$ \\
\hline${ }^{2} E_{6}(q)$ & $Z_{(3, q+1): Z_{2 f}}$ \\
\hline $\begin{array}{l}\mathrm{M}_{11}, \mathrm{M}_{23}, \mathrm{M}_{24}, \mathrm{~J}_{1}, \mathrm{~J}_{4}, \mathrm{Ru}, \mathrm{Ly}, \\
\mathrm{Co}_{1}, \mathrm{Co}_{2}, \mathrm{Co}_{3}, \mathrm{Fi}_{23}, \mathrm{Th}, \mathrm{BM}, \mathrm{M}\end{array}$ & 1 \\
\hline $\begin{array}{l}\mathrm{M}_{12}, \mathrm{M}_{22}, \mathrm{~J}_{2}, \mathrm{~J}_{3}, \mathrm{HS}, \mathrm{Suz}, \mathrm{McL}, \\
\mathrm{He}, \mathrm{O}^{\prime} \mathrm{N}, \mathrm{Fi}_{22}, \mathrm{Fi}_{24}^{\prime}, \mathrm{HN}\end{array}$ & $Z_{2}$ \\
\hline
\end{tabular}

$\operatorname{Out}\left(G_{0}\right) / N$, and $N \cong Z_{a}, H \cong Z_{b}$ for some positive integers $a$ and $b$ greater than 1 . Then $K$ has a cyclic normal subgroup, namely $N \cap K \leqslant N \cong Z_{a}$, and

$$
K /(N \cap K) \cong(N K) / N \leqslant \operatorname{Out}\left(G_{0}\right) / N \cong Z_{b}
$$


By Lemma 3.3, either $\delta_{G}(A)=1$ or $A$ is central and $\delta_{G}(A)=2$, contradicting $d(G)>2$.

In the remainder of the proof, we will use Lemma 3.3 implicitly. Suppose that $\operatorname{Out}\left(G_{0}\right)$ has shape $\left(Z_{a}: Z_{b}\right): Z_{c}$. We can assume that $G_{0} \neq E_{6}(q)$ as otherwise $\delta_{G}(A)=1$ or $A$ is a central chief factor of $G$ and $\delta_{G}(A)=2$, contradicting $d(G)>2$. Hence, $G_{0}=\mathrm{L}_{n}(q)$ where $n \geqslant 3$, or $G_{0}=\mathrm{P} \Omega_{2 m}^{+}(q)$ where $m>4, q$ is odd and $D(Q)$ is not a square. If $G_{0}=\mathrm{L}_{n}(q)$, then $\operatorname{Out}\left(G_{0}\right)=\left(Z_{(n, q-1)}: Z_{f}\right): Z_{2}$ and we may assume that $p$ is odd, $n$ and $f$ are both even and $A \cong Z_{2}$; if not, then either $\delta_{G}(A)=1$ or $A$ is a central chief factor of $G$ and $\delta_{G}(A)=2$, contradicting $d(G)>2$. Similarly, if $G_{0}=\mathrm{P} \Omega_{2 m}^{+}(q)$, then $\operatorname{Out}\left(G_{0}\right)=Z_{2} \times Z_{2} \times Z_{f}, f$ must be even and $A \cong Z_{2}$. The result follows in these cases.

Suppose that $G_{0}=\mathrm{P} \Omega_{8}^{+}(q)$. Note that $q$ is odd as otherwise Out $\left(G_{0}\right)=\operatorname{Sym}_{3} \times$ $Z_{f}$ and either $\delta_{G}(A)=1$ or $A$ is central and $\delta_{G}(A)=2$, contradicting $d(G)>2$. In particular, $\operatorname{Out}\left(G_{0}\right)=\operatorname{Sym}_{4} \times Z_{f}$. Also $f$ is even and $A \cong Z_{2}$ as otherwise $d(G)=2$, a contradiction. The result follows in this case.

Suppose finally that $G_{0}=\mathrm{P} \Omega_{2 m}^{ \pm}(q) \neq \mathrm{P} \Omega_{8}^{+}(q)$, where $m \geqslant 4, q$ is odd and $D(Q)$ is a square. Then $\operatorname{Out}\left(G_{0}\right)=D_{8} \times Z_{f}$. Again, $f$ must be even and $A \cong Z_{2}$ as otherwise $d(G)=2$, a contradiction. Once again, the result follows.

This completes our preparations. We will now prove Theorem 2.7 by considering each possibility for $G_{0}$ in turn, using the classification of finite simple groups.

\section{Almost simple groups with sporadic socle}

In this section, we prove Theorem 2.7 in the case when $\operatorname{soc}(G)$ is a sporadic simple group. We remark that the bound $d(H) \leqslant 3$ is proved directly in [10].

We first need a lemma. For a finite group $H$ and an $H$-module $V$, we will write $\operatorname{Rad}_{H}(V)$ (or $\operatorname{Rad}(V)$ if $H$ is understood from the context) to denote the $H$-radical of $V$. That is, $\operatorname{Rad}_{H}(V)$ denotes the intersection of the maximal $H$ submodules of $V$.

LEMMA 4.1. Let $H$ be a finite group, and let $N$ be an elementary abelian normal subgroup of $H$. Then $\operatorname{Rad}_{H}(N) \leqslant \operatorname{Frat}(H)$.

Proof. Suppose that $M$ is a maximal subgroup of $H$ not containing $R:=$ $\operatorname{Rad}_{H}(N)$. Then $H=R M$, so $N=R M \cap N=R(M \cap N)$. This is a contradiction since $R$ is contained in every maximal submodule of $N$, so $R$ cannot be supplemented in $N$. The claim follows. 
We are now ready to prove Theorem 2.7 for maximal subgroups of almost simple groups with sporadic socle.

Proposition 4.2. Let $G$ be an almost simple group with sporadic socle $G_{0}$. Let $H$ be a maximal subgroup of $G$, and let $A$ be a non-Frattini chief factor of $H$. Then $\delta_{H}(A) \leqslant 3$, with equality only if $A$ is central.

Proof. If $G_{0} \notin\left\{\mathrm{HS}, \mathrm{Co}_{1}, \mathrm{HN}, \mathrm{Fi}_{23}, \mathrm{Fi}_{24}^{\prime}, \mathrm{B}, \mathrm{M}\right\}$, then the chief factors of the group $H$ can be determined by using Magma [7] together with information from the Web Atlas [53]. We now deal with the remaining cases.

Suppose first that $G_{0}=\mathrm{Co}_{1}$. Then $G=G_{0}$, and $G$ has 22 conjugacy classes of maximal subgroups. In most cases, the required bounds on $\delta_{H}(A)$ follow immediately by inspection. The cases where the bounds are not immediate are when $H$ has one of the following shapes:

$$
\begin{gathered}
2^{2+12}:\left(\mathrm{Alt}_{8} \times \mathrm{Sym}_{3}\right), 2^{4+12} \cdot\left(\operatorname{Sym}_{3} \times 3 \cdot \mathrm{Sym}_{6}\right), \\
3^{6}:\left(2 \cdot \mathrm{M}_{12}\right), 3^{1+4}:\left(2 \cdot \mathrm{PSp}_{4}(3) \cdot 2\right), 3^{3+4}:\left(2 \cdot\left(\mathrm{Sym}_{4} \times \mathrm{Sym}_{4}\right)\right) .
\end{gathered}
$$

In these cases, we need more specific arguments. In particular, in what follows, we view the double cover 2. $G$ as a subgroup of $\mathrm{GL}_{24}(2)$ and use the analysis in [48].

Suppose first that $H$ has shape $2^{2+12}:\left(\mathrm{Alt}_{8} \times \mathrm{Sym}_{3}\right)$. Then, viewing $2 . G$ as a subgroup of $\mathrm{GL}_{24}(2)$, we have that $2 . H$ preserves a decomposition of the natural module $\left(\mathbb{F}_{2}\right)^{24}$ into a direct sum of three subspaces of dimension 8 . With this action, we have that $2 . H \leqslant R 2 \mathrm{Sym}_{3}$ where $R$ has shape $2_{+}^{1+6} \cdot \mathrm{O}_{6}^{+}(2)$, by [48]. Let $E$ denote the extraspecial normal subgroup $2_{+}^{1+6}$ of $R$. Then the intersection of $2 . H$ with the natural copy of $E^{3}$ in $R^{3}$ is the set of elements $\left(g_{1}, g_{2}, g_{3}\right) \in E^{3}$ with the property that $g_{1} g_{2} g_{3}$ is in the diagonal subgroup of $Z(E)^{3}$. In particular, it has order $2^{3+12}$ and is a subdirect product in $E^{3}$. Since $2 . H \cap R^{3}$ modulo this normal 2-subgroup is the diagonal subgroup of $\mathrm{O}_{6}^{+}(2)$ acted upon trivially by $\mathrm{Sym}_{3}$, we have that the three central normal factors of $2^{3+12}$ are Frattini, while the $2^{12}$ on top is a completely reducible $\left(2 . H \cap R^{3}\right)$-module with two irreducible summands each of order $2^{6}$. Since these factors are clearly conjugate under the action of $\mathrm{Sym}_{3}$, we deduce that $H$ has non-Frattini chief factors $2^{12}, \mathrm{Alt}_{8}, \mathrm{Alt}_{3}$ and $\mathrm{Sym}_{3} / \mathrm{Alt}_{3}$. Whence, $\delta_{H}(A)=1$.

Suppose next that $H$ has shape $2^{4+12} \cdot\left(\mathrm{Sym}_{3} \times 3 . \mathrm{Sym}_{6}\right)$. Then $2 . H$ preserves a decomposition of the natural module $\left(\mathbb{F}_{2}\right)^{24}$ into a direct sum of six subspaces of dimension 4 , and $2 . H \leqslant R 2 \operatorname{Sym}_{6}$ where $R$ has shape $2_{+}^{1+4} \cdot\left(\operatorname{Sym}_{3} \times 3\right)$. Defining $E$ as the $2_{+}^{1+4}$ normal subgroup of $R$, we have that $2 . H$ intersects $E^{6}$ in a subgroup of order $2^{5+12}$. As before, the $2^{5}$ at the bottom of this normal subgroup is contained 
in the Frattini subgroup of 2.H. Furthermore, the group $\left(2 . H \cap R^{6}\right) /\left(2 . H \cap E^{6}\right)$ is a subdirect product of $\left(\operatorname{Sym}_{3} \times 3\right)^{6}$. This group $\operatorname{Sym}_{3} \times 3$ acts on $E / Z(E) \cong$ $2^{4}$ completely reducibly, with $E / Z(E)$ decomposing as a direct sum of two submodules of dimension 2 . It follows that $\left(2 . H \cap R^{6}\right) /\left(2 . H \cap E^{6}\right)$ acts completely reducibly on $E^{6} / Z(E)^{6}$, with $E^{6} / Z(E)^{6}$ decomposing as a direct sum of two 12dimensional submodules. Since $2 . H /\left(2 . H \cap R^{6}\right) \cong \mathrm{Sym}_{6}$, we then have that as a $2 . H$-module, $E^{6} / Z(E)^{6}$ is a direct sum of two submodules of dimension 12 . It follows that $\left(2 . H \cap E^{6}\right) /\left(2 . H \cap Z(E)^{6}\right) \cong 2^{12}$ is irreducible as a $2 . H$-module. It follows that the non-Frattini chief factors of $H$ are $2^{12}, 3$ (the 3 in $3 . \mathrm{Sym}_{6}$ is Frattini; see [48]), 2 (with multiplicity 2 ) and Alt $_{6}$. Hence, $\delta_{H}(A) \leqslant 2$.

Using [48] and the embedding 2.G $\rightarrow \mathrm{GL}_{24}(2)$ again, we can argue in a similar way in the remaining cases. For example, suppose that $H$ has shape $3^{3+4}:\left(2 .\left(\operatorname{Sym}_{4} \times \operatorname{Sym}_{4}\right)\right)$. Then $2 . H$ preserves a decomposition of $\left(\mathbb{F}_{2}\right)^{24}$ into a direct sum of eight three-dimensional submodules. This time, the stabilizer of a three-dimensional submodule induces $3^{1+2} \cdot \mathrm{GL}_{2}(3)$, where the $\mathrm{GL}_{2}(3)$ acts naturally on the quotient of the normal extraspecial 3 -subgroup of order $3^{1+2}$ by its centre. We again deduce that the normal subgroup of order $3^{3}$ in $H$ is Frattini. Furthermore, it is shown in [48] that the $3^{4}$ on top of $O_{3}(H)$ splits as a direct sum of two irreducible $H$-modules of dimension 2. Also, $H / O_{3}(H) \cong$ 2. $\left(\operatorname{Sym}_{4} \times \operatorname{Sym}_{4}\right)$ is a central product in $\mathrm{GL}_{2}(3) \times \mathrm{GL}_{2}(3)$. Hence, the Frattini chief factors of $H$ are $3^{2}$ (of multiplicity at most 2), $2^{2}$ (of multiplicity 2), 3 (of multiplicity 2 ) and 2 (of multiplicity 2 ). Hence, $\delta_{H}(A) \leqslant 2$. (Note that we do not need to check whether or not the two chief factors of order $3^{2}$ are Frattini or not.)

Next, suppose that $G=\mathrm{Fi}_{23}$. The maximal subgroups of $G$ are determined in [32]. The group $G$ has 14 conjugacy classes of maximal subgroups. If

$$
H \notin\left\{3^{1+8} \cdot 2^{1+6} \cdot 3^{1+2} \cdot 2 \cdot \operatorname{Sym}_{4},\left[3^{10}\right] \cdot\left(\mathrm{L}_{3}(3) \times 2\right), 2^{6+8} \cdot\left(\mathrm{Alt}_{7} \times \mathrm{Sym}_{3}\right)\right\},
$$

then the result follows by using Magma, together with the information in the Web Atlas [53]. We now deal with the outstanding cases. Suppose first that $H$ has shape $3^{1+8} \cdot 2^{1+6} \cdot 3^{1+2} \cdot 2 \mathrm{Sym}_{4}$. The central subgroups in the extraspecial sections $3^{1+8}, 2^{1+6}$ and $3^{1+2}$ are Frattini, so we just need to determine the action of $H$ on the elementary abelian factors of these sections by their central subgroup. Information on the structure of $H$ is given in [50, Section 1.2], and from this, we deduce that $H / 3^{1+8}$ acts faithfully on the $3^{8}$. The group $H / 3^{1+8}$ preserves a decomposition of the $3^{8}$ into a direct sum of four subspaces, each of dimension 2. Furthermore, the stabilizer of one of these subspaces induces either $\mathrm{SL}_{2}(3)$ or $\mathrm{GL}_{2}(3)$ on the subspace, and $H / 3^{1+8}$ induces $\mathrm{Alt}_{4}$ on the set of subspaces. It follows that $H$ acts irreducibly on the $3^{8}$. One can also see from [50, Section 1.2] that the action of $H$ on the $2^{6}$ is irreducible (it is, in fact, a tensor product of a fully deleted permutation module for $\mathrm{Sym}_{4}$ with the natural module for $Z_{3} \leqslant \mathrm{GL}_{2}(2)$ ). Finally, 
$2 \mathrm{Sym}_{4} \cong \mathrm{GL}_{2}$ (3) acts naturally on the $3^{2}$ in $3^{1+2}: 2 \mathrm{Sym}_{4}$, so we deduce that the non-Frattini chief factors of $H$ are $3^{8}, 2^{6}, 3^{2}, 2^{2}, 3,2$ (the centre of $2 \mathrm{Sym}_{4} \cong$ $\mathrm{GL}_{2}(3)$ is Frattini). Hence, $\delta_{H}(A)=1$ for all $A$.

Suppose next that $H$ has shape $3^{3} \cdot 3^{7} \cdot\left(\mathrm{L}_{3}(3) \times 2\right)$. The $3^{3}$ normal subgroup $U$ on the bottom is (up to conjugation in $G$ ) contained in the $3^{1+8}$ extraspecial subgroup $E$ of $G$ from the previous paragraph. Furthermore, $U$ contains $Z(E)$ and is acted upon irreducibly by $H$ (see [51, Proposition 4.2]). Now, as argued in [50, Page 81], the group $\mathrm{L}_{3}(3) \cong \mathrm{SL}_{3}(3)$ acts on the elementary abelian group $F:=E / U$ as a direct sum of two copies of the natural module of dimension 3 . Furthermore, it follows immediately from [51, Appendix B] that the group $Z_{2}$ on top acts by permuting the two $\mathrm{L}_{3}(3)$-summands in $F$. We deduce that $H$ has chief factors $3^{3}, 3^{6}, 3, \mathrm{~L}_{3}(3), 2$, and, hence, $\delta_{H}(A)=1$.

Finally, assume that $H$ has shape $2^{6+8} \cdot\left(\mathrm{Alt}_{7} \times \mathrm{Sym}_{3}\right)$. By [22], $H$ has a subgroup of index 3 of shape $2^{7+4} \cdot\left(2^{4}\right.$. Alt 7$) \leqslant 2^{11} \cdot \mathrm{M}_{23}$, where the group $2^{4}$. Alt on top is a maximal subgroup of $\mathrm{M}_{23}$. The $2^{11}$ on the bottom is an irreducible $\mathrm{M}_{23}$-module, and we can use the information in [53] to deduce that $2^{11}$ has three composition factors as an $\mathrm{Alt}_{7}$-module, of dimensions 1, 6 and 4. Since the $2^{4}$ in $2^{4}$. Alt $_{7}<\mathrm{M}_{23}$ is also irreducible as an $\mathrm{Alt}_{7}$-module, we deduce that $\delta_{H}(A) \leqslant 2$.

Next, assume that $G \in\left\{\mathrm{Fi}_{24}^{\prime}, \mathrm{Fi}_{24}\right\}$. The conjugacy classes of maximal subgroups of $G$ are determined in [36], and the required bounds on $\delta_{H}(A)$ are immediate in most cases. However, the 2-local and 3-local maximal subgroups of $G$ need special attention and we use information on their structure given in [50] to obtain the required bounds on $\delta_{H}(A)$. For example, suppose that $G=\mathrm{Fi}_{24}^{\prime}$ and that $H$ has shape $2_{+}^{1+12} \cdot 3 . \mathrm{U}_{4}(2): 2$. Then the normal extraspecial 2-group $E$ of order $2^{1+12}$ contains a Frattini chief factor $Z(E)$ of $H$ of order 2 and a chief factor $E / Z(E)$ of order $2^{12}$. This is clear from [50, Section 3]. Indeed, viewing $E / Z(E)$ as a six-dimensional space over $\mathbb{F}_{4}$, the group 3. $\mathrm{U}_{4}(2)$ embeds as an irreducible subgroup of $\mathrm{GL}_{6}(4)$. The 2 on the top acts on $E / Z(E)$ and $3 . \mathrm{U}_{4}(2)$ as a field automorphism of $\mathbb{F}_{4}$, and this implies that $E / Z(E)$ is irreducible as a module over $\mathbb{F}_{2}$, as claimed. We deduce that $H$ has non-Frattini chief factors of order $2^{12}, 3, \mathrm{U}_{4}(2)$ and 2 , and hence $\delta_{H}(A) \leqslant 2$.

As another example, let us take $H$ to have shape $3^{2} \cdot 3^{4} \cdot 3^{8} \cdot\left(\mathrm{Alt}_{5} \times 2 \mathrm{Alt}_{4}\right): 2$. The induced actions of $\mathrm{H} / \mathrm{O}_{3}(\mathrm{H}) \cong\left(\mathrm{Alt}_{5} \times 2 \mathrm{Alt}_{4}\right): 2$ (which is a subdirect product of $\operatorname{Sym}_{5} \times \mathrm{GL}_{2}(3)$ ) on the elementary abelian factor groups $3^{2}, 3^{4}$ and $3^{8}$ can be deduced from [50, Section 2]. In particular, we get that the $3^{4}$ is the fully deleted permutation module for $\mathrm{Sym}_{5}$ over $\mathbb{F}_{3}$, while the $3^{8}$ is a tensor product of the fully deleted permutation module for $\mathrm{Sym}_{5}$ and the natural module for $2 \mathrm{Alt}_{4} \cong \mathrm{SL}_{2}$ (3). In particular, the $3^{8}$ is an irreducible $H$-module. The $3^{2}$ normal subgroup is also an irreducible $H$-module, and we deduce that $H$ has (possible) non-Frattini chief factors $3^{2}, 3^{4}, 3^{8}, \mathrm{Alt}_{5}, 2^{2}, 3,2$. Whence, $\delta_{H}(A)=1$. 
Next, suppose that $G \in\{\mathrm{HN}, \mathrm{HN}: 2\}$. The maximal subgroups of $G$ are determined in [44]. Again, the required bounds on $\delta_{H}(A)$ follow immediately in most cases, though there are some local maximal subgroups for which more care is needed. In these cases, the structure of $H$ is given in [44, Section 3], and the bounds can then be deduced. For example, if $G=\mathrm{HN}: 2$ and $H$ has shape $2^{3} \cdot 2^{2} \cdot 2^{6} \cdot\left(\operatorname{Sym}_{3} \times \mathrm{L}_{3}(2)\right)$, then the $2^{3}$ normal subgroup is contained in $\operatorname{Sym}_{12} \leqslant G$ and acts regularly on eight letters and trivially on the remaining four letters. Thus, the normalizer of $2^{3}$ in $\operatorname{Sym}_{12}$ has shape $2^{3} . \mathrm{L}_{3}(2) \times \mathrm{Sym}_{4}$. It follows that the $2^{3}$ and $2^{2}$ factors are irreducible as $H$-modules, with induced actions $\mathrm{L}_{3}(2)$ and $\mathrm{Sym}_{3} \cong \mathrm{GL}_{2}(2)$, respectively. Finally, the $2^{6}$ factor is a quotient of the extraspecial subgroup $2_{+}^{1+8}$ of $\mathrm{HN}$ and splits as a direct sum of two threedimensional submodules, with induced action $\mathrm{Sym}_{4} \leqslant O_{4}^{+}(4)$. The other cases are easier and they are handled in the same way, using [44, Section 3] as above.

Finally, we deal with the cases $G \in\{\mathrm{BM}, \mathrm{M}\}$. Since 2 . BM $\leqslant \mathrm{M}$, the bounds on $\delta_{H}(A)$ in the case $G=\mathrm{BM}$ will quickly follow from our work in the case $G=\mathrm{M}$. We will make some further remarks about this once we have dealt with the $G=$ M case.

So assume that $G=\mathrm{M}$. At this stage, 44 conjugacy classes of maximal subgroups $H$ of $G$ have been identified, namely the 43 in [53] and the one with representative $\mathrm{L}_{2}(41)$ (see [45]). Moreover, any further, if any, maximal subgroup of $G$ is almost simple with socle $\mathrm{L}_{2}(13), \mathrm{U}_{3}(4), \mathrm{U}_{3}(8)$ or ${ }^{2} B_{2}(8)$. By Proposition 3.12, the required bounds on $\delta_{H}(A)$ follow when $H$ is almost simple. Also, for 37 of the 43 representatives $H$ in [53], the information in [53], together with [7], allow us to determine the action of $H$ on each of its chief factors, and hence the required bounds on $\delta_{H}(A)$. The remaining representatives are the following:

(1) 2. BM

(3) $2^{10+16} \cdot \Omega_{10}^{+}(2)$

(5)
(2) $2^{1+24} \cdot \mathrm{Co}_{1}$

(4) $2^{3+6+12+18} \cdot\left(\mathrm{L}_{3}(2) \times 3 \mathrm{Sym}_{6}\right)$

In the first case, $H$ is quasisimple, so $\delta_{H}(A)=1$ by Lemma 3.5. In case (2), the group $\mathrm{Co}_{1}$ acts (irreducibly) on the Frattini quotient of $2_{+}^{1+24}$ as it acts on the Leech lattice modulo 2 (see [41, Section 2]). In particular, $H$ has non-Frattini chief factors $2^{24}$ and $\mathrm{Co}_{1}$, and the result follows. In case (3), the normal $2^{10}$ subgroup is acted upon irreducibly by $H$, while the normal $2^{16}$ section has a $K$ composition series consisting of two nonisomorphic $K$-modules of dimension 8, for a subgroup $K<H$. This follows from [41, Corollary 5.5 and Lemma 5.6]. The required bounds on $\delta_{H}(A)$ are then immediate.

Suppose now that we are in case (4). By [41, Lemmas 4.2 and 4.8], the $2^{3}$ and $2^{6}$ normal sections in $H$ are irreducible $H$-modules. To determine the actions of 
$H$ on the remaining two sections, we view the normal subgroup $E \cong 2^{3}$ as a subgroup in the extraspecial subgroup $Q \cong 2_{+}^{1+24} \leqslant G$. The normalizer of $Q$ in $G$ has shape $Q \cdot \mathrm{Co}_{1}$. Let $E_{0}$ be a two-dimensional subspace of $E$ containing $Z(Q)$. Then by [41, Section 2 and Lemma 4.5], the centralizer of $E_{0}$ in $N:=N_{G}(Q)$ has shape $2^{2} \cdot T .\left(T^{*} \times T^{*}\right): \mathrm{M}_{24}$, where $T \cong 2^{11}$ denotes the Todd module for $\mathrm{M}_{24}$ and $T^{*}$ denotes its dual (see [27] for another proof of this fact). By [41, Lemma 4.8], $C:=C_{N}(E) \leqslant C_{N}\left(E_{0}\right)$ reduces to the group $K:=2^{6}: 3 \mathrm{Sym}_{6} \leqslant \mathrm{M}_{24}$ modulo $O_{2}\left(C_{N}\left(E_{0}\right)\right)$. One can now check, using [7, 53], that the action of $K$ on the Todd module for $\mathrm{M}_{24}$ (referred to as the Golay cocode in [53]) has composition factors $A_{1}, A_{6}$ and $A_{4}$ of dimensions 1,6 and 4, respectively. Its radical quotient $T / \operatorname{Rad}_{K}(T)$ is irreducible of dimension 4. The dual modules $A_{1}^{*}=A_{1}, A_{6}^{*} \neq A_{6}$ and $A_{4}^{*} \neq A_{4}$ to these modules then give the composition factors in the dual to the Todd module for $\mathrm{M}_{24}$ (referred to as the Golay code in [53]). We also have that $T^{*} / \operatorname{Rad}_{K}\left(T^{*}\right)$ is irreducible, this time with dimension 1 . Thus, $C / 2^{3}$ has shape $A_{6} . A_{4} .\left(A_{4}^{*} \cdot A_{6}^{*} \cdot 2 \times A_{4}^{*} \cdot A_{6}^{*} \cdot 2\right) \cdot 2^{6}: 3 \mathrm{Sym}_{6}$, and the non-Frattini chief factors within are $A_{4}, 2,2$, using Lemma 4.1. The final action we need to check is the action of the $3 \mathrm{Sym}_{6}$ on the $2^{6}$ normal subgroup of the top group. Using Magma [7], we quickly see that the action is irreducible. The bound $\delta_{H}(A) \leqslant 3$ is now immediate.

Finally, assume that we are in case (5), and denote by $E_{5}$ the normal subgroup of $H$ of order $2^{5}$. By [41, Lemma 5.10], $E_{5}$ is contained in precisely three conjugates of the normal $2^{10}$ subgroup from case (3). Let $B_{1}$ and $B_{2}$ be two such conjugates, and let $R=B_{1} B_{2}$. Then $R$ is elementary abelian of order $2^{15}, B_{3} \leqslant R$ and $B_{1} \cap B_{2}=E_{5}$, by [41, Lemma 6.11]. Hence, $R \unlhd H=N_{G}\left(E_{5}\right)$. We are now ready to determine the $H$-chief factors contained in $R$. First, [41, Lemma 4.2] implies that $E_{5}$ is a chief factor of $H$, with $H / C_{H}\left(E_{5}\right) \cong \mathrm{L}_{5}(2)$. Next, $R / E_{5}$ contains a two-dimensional subspace $W$ which is acted upon as a fully deleted permutation module by $N_{H}(W) / C_{H}(W) \cong \operatorname{Sym}_{3}$. Also, $K:=N_{N_{G}\left(B_{1}\right)}\left(E_{5}\right)$ induces the maximal parabolic subgroup $2^{10}$. $\mathrm{L}_{5}(2) \leqslant \Omega_{10}^{+}(2)$ on $B_{1}$, by [41, Proof of Lemma 6.11]. It follows from this and [41, Lemma 6.9] that $R / E_{5} \cong 2^{10}$ has two $K$-composition factors, each of dimension 5. It follows immediately that $R / E_{5}$ is irreducible as an $H$-module. Thus, all that remains is to determine the $H$-chief factors in $O_{2}(H) / R \cong 2^{20}$. First, as mentioned above, $K=N_{N_{G}\left(B_{1}\right)}\left(E_{5}\right)$ induces the maximal parabolic subgroup $2^{10} . \mathrm{L}_{5}(2) \leqslant \Omega_{10}^{+}(2)$ on $B_{1}$, and hence on $N_{N_{G}\left(B_{1}\right)}\left(E_{5}\right) / B_{1} \cong 2^{16}$. Thus, we need to determine the composition factors for the restriction of the irreducible 16-dimensional representation $U$ for $\Omega_{10}^{+}(2)$ over $\mathbb{F}_{2}$ to the subgroup $2^{10} \cdot \Omega_{5}^{+}(2)$. By [41, Lemma 6.4], the group $\Omega_{10}^{+}(2)$ has two orbits in its action on the nonzero elements of $U$, of sizes 2295 and 63240. Furthermore, [41, Lemma 6.9] implies that $U$ has a five-dimensional $K$ submodule. By constructing the transitive permutation representations of $\Omega_{10}^{+}(2)$ of degrees 2295 and 63240 and the induced action of the group $K<\Omega_{10}^{+}(2)$ 
in these representations, we quickly deduce that the only possibility is that $K$ has orbits of sizes $31,1024,1240,3720,27776$ and 31744 and $U$ has $K$-composition factors of dimensions 5, 10 and 1. Furthermore, $U$ and $O_{2}(K)$ are nonisomorphic as $K$-modules, and $\mathrm{O}_{2}(K)$ is irreducible (one can see this by computing the orbits of $K$ in its action on the nonidentity elements of $O_{2}(K)$ ). Thus, we have that the $2^{20}$ section in $H$ is either irreducible or consists of two nonisomorphic $H$-chief factors, each of order $2^{10}$. Thus, an $H$-chief factor of order $2^{5}$ occurs at most once in $O_{2}(H)$, while there is at most one pair of $H$-equivalent chief factors of order $2^{10}$. Since $H / O_{2}(H) \cong \operatorname{Sym}_{3} \times \mathrm{L}_{5}(2)$, we deduce that $\delta_{H}(A) \leqslant 2$ in all cases, and this gives us what we need.

This completes the analysis for the case $G=\mathrm{M}$. If $G=\mathrm{BM}$, then the work above suffices to yield the result for most maximal subgroups $H$ of $G$ for which the information in the Web Atlas is insufficient to prove the required bounds on $\delta_{H}(A)$. For example, suppose that $H$ has shape $\left[2^{35}\right] .\left(\operatorname{Sym}_{5} \times \mathrm{L}_{3}(2)\right)$. Then $2 . H$ is a subgroup of the maximal subgroup $L$ of $\mathrm{M}$ of shape $2^{3+6+12+18} \cdot\left(\mathrm{L}_{3}(2) \times 3 \mathrm{Sym}_{6}\right)$. We have already seen above that the group $\mathrm{L}_{3}(2)$ acts on the $2^{3}$ normal subgroup at the bottom of $L$ irreducibly, and this is the same action as the $\mathrm{L}_{3}(2)$ section of $H$ on the $2^{3}$ there. Now, as above, it is convenient to view the normal subgroup 2. $\left(\mathrm{O}_{2}(\mathrm{H}) . \mathrm{Sym}_{5}\right)$ of $2 . \mathrm{H}$ as a subgroup of

$$
2^{2} \cdot T \cdot\left(T^{*} \times T^{*}\right) \cdot \mathrm{M}_{24},
$$

where $T$ denotes the Todd module for $\mathrm{M}_{24}$ and $T^{*}$ denotes its dual. Viewing $R:=2^{6} . \mathrm{Sym}_{5}$ as a subgroup of $\mathrm{M}_{24}$, we can use Magma to determine the actions of $R$ on $T$ and $T^{*}$. We find that for any $R$-submodule $S$ of $T$ or $T^{*}$, the $R$-radical quotient $S / \operatorname{Rad}_{R}(S)$ of $S$ is irreducible of dimension either 1 or 4 . Furthermore, the radical quotient of the $2^{6}$ section in $R$ is irreducible of dimension 4 . Using Lemma 4.1, we deduce that there are at most four non-Frattini $H$-chief factors contained in $\mathrm{O}_{2}(H)$, and at most three of these have dimension 1. Hence, $\delta_{H}\left(Z_{2}\right) \leqslant 3$. Moreover, if more than two chief factors of order $2^{4}$ occur, then at most two of them, say $A_{1}$ and $A_{2}$, are isomorphic to $A$, and the others are isomorphic to the dual $A^{*}$. The other cases are entirely similar.

\section{Almost simple groups with alternating socle}

In this section, we prove the following.

Proposition 5.1. Suppose that $G_{0} \leqslant G \leqslant \operatorname{Aut}\left(G_{0}\right)$, where $G_{0}=\operatorname{Alt}_{n}$ is an alternating group of degree $n \geqslant 5$. Let $H$ be a maximal subgroup of $G$ and let $A$ be a non-Frattini chief factor of $H$.

(i) If $A$ is nonabelian, then $\delta_{H}(A)=1$. 
(ii) If $A$ is abelian but noncentral, then $\delta_{H}(A) \leqslant 2$.

(iii) If $A$ is central, then $\delta_{H}(A) \leqslant 4$. Moreover, if $\delta_{H}(A) \in\{3,4\}$, then $|A|=2$. Finally, $\delta_{H}(A)=4$ if and only if $G \in\left\{\operatorname{Alt}_{n}, \operatorname{Sym}_{n}\right\}, H=\left(T^{k} \cdot(\operatorname{Out}(T) \times\right.$ Sym $\left.\left._{k}\right)\right) \cap G$ is of diagonal type (that is, $n=|T|^{k-1}$ where $T$ is nonabelian simple and $k>1), \operatorname{Sym}_{k} \leqslant H,|A|=2, A$ is a section of $\operatorname{Out}(T) \times \operatorname{Sym}_{k}$ and $\delta_{\mathrm{Out}(T) \cap\left(H / T^{k}\right)}(A)=3$.

We remark that the bound $d(H) \leqslant 4$, with equality if and only if we are in the exceptional case in part (iii) above, is proved in [10, Proposition 4.2].

Recall that if $n \neq 6$, then $G=\operatorname{Alt}_{n}$ or $G=\operatorname{Sym}_{n}$, whereas if $n=6$, then $G \in\left\{\mathrm{Alt}_{6}, \mathrm{Sym}_{6}, \mathrm{M}_{10}, \mathrm{PGL}_{2}(9), \mathrm{P} \Gamma \mathrm{L}_{2}(9)\right\}$.

We first treat the case where $G$ is neither an alternating group nor a symmetric group. In particular, $n=6$ and $G \in\left\{\mathrm{M}_{10}, \mathrm{PGL}_{2}(9), \mathrm{P} \Gamma \mathrm{L}_{2}(9)\right\}$. We use Magma to check our calculations.

Lemma 5.2. Suppose that $G \in\left\{\mathrm{M}_{10}, \mathrm{PGL}_{2}(9), \mathrm{P} \Gamma \mathrm{L}_{2}(9)\right\}$. Let $H$ be a maximal subgroup of $G$ and let $A$ be a non-Frattini chief factor of $H$. Then $\delta_{H}(A) \leqslant 3$, with $\delta_{H}(A) \leqslant 1$ if $A$ is nonabelian. Moreover, $\delta_{H}(A)=3$ only if $|A|=2$.

We can now suppose that $G=\mathrm{Alt}_{n}$ or $G=\operatorname{Sym}_{n}$, where $n \geqslant 5$. We recall the O'Nan-Scott theorem which describes the maximal subgroups of $G$.

Theorem 5.3 [3, Appendix]. Let $G=\mathrm{Alt}_{n}$ or $G=\mathrm{Sym}_{n}$, where $n \geqslant 5$. Let $H$ be a maximal subgroup of $G$. One of the following assertions holds:

(i) $H$ is intransitive: $H=\left(\operatorname{Sym}_{k} \times \operatorname{Sym}_{n-k}\right) \cap G$ where $1 \leqslant k<n / 2$.

(ii) $H$ is affine: $H=\operatorname{AGL}_{d}(p) \cap G$ where $n=p^{d}$, $p$ is prime and $d \geqslant 1$.

(iii) $H$ is imprimitive or of wreath type: $H=\left(\operatorname{Sym}_{k} 2 \operatorname{Sym}_{t}\right) \cap G$ where $n=k t$ or $n=k^{t}$ for some $t>1$.

(iv) $H$ is of diagonal type: $H=\left(T^{k}\right.$.(Out $\left.\left.(T) \times \operatorname{Sym}_{k}\right)\right) \cap G$ where $T$ is nonabelian simple and $n=|T|^{k-1}$ for some $k>1$.

(v) $H$ is almost simple.

We can now finish the proof of Proposition 5.1. We remark that the proof here is similar to the proof of [10, Proposition 4.2].

Proof of Proposition 5.1. By Proposition 3.12, if $H$ is almost simple, then $\delta_{H}(A) \leqslant 3$ with equality only if $A \cong Z_{2}$ is central. We therefore assume in the 
remainder that $H$ is not almost simple. Suppose first that $G=\operatorname{Sym}_{n}$. Suppose $H$ is intransitive. The chief factors of $H$ are then $\mathrm{Alt}_{k}, \mathrm{Alt}_{n-k}, Z_{2}$ and $Z_{2}$. In particular, either $A$ is a nonabelian and $\delta_{H}(A)=1$ or $A \cong Z_{2}$ is a central chief factor of $H$ satisfying $\delta_{H}(A) \leqslant 2$.

Suppose now that $H$ is affine. Then $H=V: \operatorname{GL}_{d}(p)$, where $V=Z_{p}^{d}$ is an elementary abelian $p$-group and is the unique minimal normal subgroup of $H$. Moreover, $H=V \cdot Z_{a} \cdot \mathrm{L}_{d}(p) . Z_{b}$, with $a=(d, p-1)$ and $b=p-1$. Since a chief factor of $H$ that is a section of $Z_{a}$ is Frattini, it follows from Lemma 3.10 that $\delta_{H}(A)=1$.

Suppose that $G$ is imprimitive or of wreath type. In the following, when we write $H:=N_{1} \cdot N_{2} \cdots N_{t-1} \cdot N_{t}=H$, we mean that $H$ has normal series with factors $N_{1}, \ldots, N_{t}$. Then by Lemma 3.9,

$$
H= \begin{cases}\operatorname{Alt}_{k}^{t} \cdot 2 \cdot 2^{t-2} \cdot 2 \cdot \mathrm{Alt}_{t} \cdot 2 & \text { if } t \text { is even } \\ \operatorname{Alt}_{k}^{t} \cdot 2 \cdot 2^{t-1} \cdot \mathrm{Alt}_{t} \cdot 2 & \text { if } t \text { is odd }\end{cases}
$$

Moreover, if $t$ is even, then the first $Z_{2}$ chief factor of $H$ in (5.1) is Frattini.

Suppose $t \neq 4$ and $k \neq 4$. By Lemma 3.9, we see that (5.1) is a chief series for $H$. In particular, $\delta_{H}(A) \leqslant 2$, and if $\delta_{H}(A)=2$, then $A \cong Z_{2}$ is central.

Suppose $t=4$ and $k \neq 4$. By Lemma 3.9, (5.1) can be refined to the following series for $H$, in which all the factors are chief factors:

$$
\mathrm{Alt}_{k}^{4} \cdot 2 \cdot 2^{2} \cdot 2 \cdot 2^{2} \cdot 3 \cdot 2
$$

Now $H$ has two chief factors $A_{1}$ and $A_{2}$ with $A_{1} \cong A_{2} \cong Z_{2} \times Z_{2}$. However, $A_{1}$ and $A_{2}$ are not $H$-isomorphic and so they are not $H$-equivalent. It follows that $\delta_{H}(A) \leqslant 2$, and if $\delta_{H}(A)=2$, then $A \cong Z_{2}$ is central.

Suppose $t \neq 4$ and $k=4$. By Lemma 3.9, a chief series of $H$ is

$$
H= \begin{cases}2^{2 t} \cdot 3^{t} \cdot 2 \cdot 2^{t-2} \cdot 2 \cdot \text { Alt }_{t} \cdot 2 & \text { if } t \text { is even } \\ 2^{2 t} \cdot 3^{t} \cdot 2 \cdot 2^{t-1} \cdot \text { Alt }_{t} \cdot 2 & \text { if } t \text { is odd }\end{cases}
$$

Again $\delta_{H}(A) \leqslant 2$, and if $\delta_{H}(A)=2$, then $A \cong Z_{2}$ is central.

Finally, if $t=k=4$, then a chief series of $H$ is given by

$$
H=2^{8} \cdot 3^{4} \cdot 2 \cdot 2^{2} \cdot 2 \cdot 2^{2} \cdot 3 \cdot 2 \text {. }
$$

Again, since the first $Z_{2}$ chief factor of $H$ is Frattini and the two $Z_{2} \times Z_{2}$ chief factors of $H$ are not $H$-equivalent, it follows that $\delta_{H}(A) \leqslant 2$, with equality only if $A \cong Z_{2}$ is central.

Suppose next that $H$ is of diagonal type so that $H=T^{k}$. $\left(\operatorname{Out}(T) \times \operatorname{Sym}_{k}\right)$, where $n=|T|^{k-1}$. Since $T^{k}$ is a chief factor of $H$ and $\delta_{H}\left(T^{k}\right)=1$, we can 
restrict our analysis to the non-Frattini chief factors $A$ of $H$ which appear as sections of $\operatorname{Out}(T) \times \operatorname{Sym}_{k}$. It follows from Proposition 3.12 that $\delta_{H}(A) \leqslant 4$. Moreover, if $\delta_{H}(A) \geqslant 3$, then $A \cong Z_{2}$ is central, and $\delta_{H}(A)=4$ if and only if $\delta_{\operatorname{Out}(T)}(A)=3$. (Note that if $k \in\{3,4\}$, then $H$ can have three chief factors $A_{1}, A_{2}$ and $B$ isomorphic to $Z_{3}$. However, if this is the case, then $A_{1}$ and $A_{2}$ appear as sections of $\operatorname{Out}(T) \geqslant\left(Z_{3} \times Z_{3}\right): 2$, whereas $B$ appears as a section of $\operatorname{Sym}_{k}$ which has a single $Z_{3}$ composition factor. In particular, for $i \in\{1,2\}$, $A_{i}$ and $B$ have nonisomorphic centralizers and so are not $H$-isomorphic. Hence, $\delta_{H}\left(Z_{3}\right) \leqslant 2$.)

Suppose finally that $G=\mathrm{Alt}_{n}$. Note that $H$ is a subgroup of index at most 2 in a subgroup of $\mathrm{Sym}_{n}$ that is of intransitive, affine, imprimitive, wreath, diagonal or almost simple type as in (i)-(v) of Theorem 5.3. If $H$ is not of diagonal type, then a similar argument to the one given in the case where $G=\operatorname{Sym}_{n}$ yields the result. Assume that $H$ is of diagonal type. There are two cases to consider, respectively, as $H$ is a subgroup of $T^{k}$.(Out $\left.(T) \times \operatorname{Sym}_{k}\right)$ of index 1 or 2 . In the former case, as before, we get $\delta_{H}(A) \leqslant 4$. Moreover, if $\delta_{H}(A) \geqslant 3$, then $A \cong Z_{2}$ is central, and $\delta_{H}(A)=4$ if and only if $\delta_{\mathrm{Out}(T)}(A)=3$. We therefore assume that $H$ is a subgroup of $T^{k}$. $\left(\operatorname{Out}(T) \times \operatorname{Sym}_{k}\right)$ of index 2 . Note that $T^{k}$ is the unique minimal normal subgroup of $H$. Let $D=\left\{\left(t_{1}, \ldots, t_{k}\right): t_{1} \in T, t_{1}=\cdots=t_{k}\right\} \cong T$ be the diagonal subgroup of $T^{k}$. Then $\operatorname{Sym}_{n}$ is the symmetric group on the set $\Omega$ of cosets of $D$ in $T^{k}$. Also $\sigma \in \operatorname{Sym}_{k}$ acts on $\Omega$ by sending a coset $D\left(t_{1}, \ldots, t_{k}\right)$ to $D\left(t_{\sigma(1)}, \ldots, t_{\sigma(k)}\right)$.

Suppose that $k \geqslant 3$. A transposition $\tau$ of $\operatorname{Sym}_{k}$ then fixes $|T|^{k-2}$ points of $\Omega$. It follows that, seen as an element of $\operatorname{Sym}_{n}, \tau$ is a product of

$$
\frac{n-|T|^{k-2}}{2}=\frac{|T|^{k-2}(|T|-1)}{2}
$$

disjoint 2-cycles. In particular, a transposition of $\mathrm{Sym}_{k}$ is an even permutation of $\operatorname{Sym}_{n}$ and $\operatorname{Sym}_{k} \leqslant \mathrm{Alt}_{n}$. Therefore, $H=T^{k}$. $\left(L \times \operatorname{Sym}_{k}\right)$, where $L$ is a subgroup of $\operatorname{Out}(T)$ of index 2. It follows from Proposition 3.12 that $\delta_{H}(A) \leqslant 4$. Moreover, if $\delta_{H}(A) \geqslant 3$, then $A \cong Z_{2}$ is central, and $\delta_{H}(A)=4$ if and only if $\delta_{\mathrm{Out}(T) \cap\left(H / T^{k}\right)}(A)=3$.

Suppose finally that $k=2$. The transposition $\tau=(1,2) \in \mathrm{Sym}_{k}$ fixes a coset $D\left(t_{1}, t_{2}\right)$ if and only if $t_{1}=t t_{2}$ for some $t \in T$ of order dividing 2. In particular, $\tau$ fixes $i_{2}(T)+1$ points of $\Omega$, where $i_{2}(T)$ is the number of involutions of $T$. It follows that, seen as an element of $\operatorname{Sym}_{n}, \tau$ is a product of

$$
N=\left(|T|-i_{2}(T)-1\right) / 2
$$

2-cycles. 
If $N$ is odd, then $\tau \notin H, H=T$.Out( $T$ ) and so, by Proposition 3.12, $\delta_{H}(A) \leqslant 3$ with equality only if $A \cong Z_{2}$ is central and $\delta_{\operatorname{Out}(T)}(A)=3$. If $N$ is even, then $H=T$. $\left(L \times Z_{2}\right)$, where $L$ is a subgroup of $\operatorname{Out}(T)$ of index 2 . It follows from Proposition 3.12 that $\delta_{H}(A) \leqslant 4$. Moreover, if $\delta_{H}(A) \geqslant 3$, then $A \cong Z_{2}$ is central, and $\delta_{H}(A)=4$ if and only if $\delta_{\mathrm{Out}(T) \cap\left(H / T^{k}\right)}(A)=3$.

\section{Almost simple groups with classical socle}

In this section, we prove Theorem 1 for maximal subgroups of classical groups. We begin by fixing some notation which will be retained for the remainder of the section. First, fix a vector space $V$ of dimension $n$ over a field $\mathbb{F}$. Also, let $\kappa: V^{l} \rightarrow \mathbb{F}$ be a map, where either $l=1$ and $\kappa$ is a nondegenerate quadratic form on $V$ (we will call this case $\mathbf{O}$ ) or $l=2$ and $\kappa$ is either the zero form (case $\mathbf{L}$ ), a nondegenerate unitary form (case $\mathbf{U}$ ), or a nondegenerate symplectic form (case S) on $V$.

Now define $I(V, \kappa) \leqslant \mathrm{GL}(V)$ to be the group of $\kappa$-isometries of $V$, define $S(V, \kappa):=I(V, \kappa) \cap \operatorname{SL}(V)$ to be the group of special $\kappa$-isometries of $V$, define $\Delta(V, \kappa)$ to be the group of $\kappa$-similarities on $V$ and define $\Gamma(V, \kappa)$ to be the group of $\kappa$-semisimilarities of $V$. Also, define $\Omega(V, \kappa)$ to be the kernel of the spinor norm on $S(V, \kappa)$ in case $\mathbf{O}$ and define $\Omega(V, \kappa):=S(V, \kappa)$ otherwise. Finally, we define another group $\Sigma$ as follows: in case $\mathbf{L}$, the group $S(V)=S(V, \kappa)$ has an automorphism $\iota$ which is defined by fixing a basis for $V$ and then composing the inverse and transpose antiautomorphisms of the group $S(V)$ viewed as a matrix group over the field $\mathbb{F}$ with respect to this basis. Set $\Sigma(V, \kappa):=\Gamma(V, \kappa)\langle\iota\rangle$ in this case, and $\Sigma(V, \kappa):=\Gamma(V, \kappa)$ otherwise. We then have a normal series

$$
\Omega(V, \kappa) \leqslant S(V, \kappa) \leqslant I(V, \kappa) \leqslant \Delta(V, \kappa) \leqslant \Gamma(V, \kappa) \leqslant \Sigma(V, \kappa) .
$$

Each group in (6.1) also normalizes the group of scalars of $I(V, \kappa)$, which we call $Z$. Writing bars to denote reduction modulo $Z$, we then get another normal series

$$
\overline{\Omega(V, \kappa)} \leqslant \overline{S(V, \kappa)} \leqslant \overline{I(V, \kappa)} \leqslant \overline{\Delta(V, \kappa)} \leqslant \overline{\Gamma(V, \kappa)} \leqslant \overline{\Sigma(V, \kappa)} .
$$

Finally, fix a prime $p$ and a power $q:=p^{f}$ of $p$. We will assume throughout that the field $\mathbb{F}$ is the field $\mathbb{F}:=\mathbb{F}_{q^{u}}$ of $q^{u}$ elements, where $u:=2$ if we are in case $\mathbf{U}$, and $u:=1$ otherwise.

Now, let $X$ be one of the symbols $\{\Omega, S, I, \Delta, \Gamma, \Sigma\}$. If two forms $\kappa$ and $\kappa^{\prime}$ are similar, then $X(V, \kappa) \cong X\left(V, \kappa^{\prime}\right)$. Thus, since there is a unique similarity class of symplectic forms and a unique similarity class of unitary forms over $\mathbb{F}$, we can omit reference to the specific form $\kappa$ and speak of the symplectic and the unitary $X$-group $X(V)=X(V, \kappa)$. When we are in case $\mathbf{O}$ with $n$ even, there are two 
similarity classes of quadratic forms on $V$, which we will refer to as case $\mathbf{O}^{+}$and case $\mathbf{O}^{-}$. (These cases are distinguished according to whether the Witt index of $\kappa$ is 0 or 1 , respectively.) When we are in case $\mathbf{O}$ with $n$ odd, there is a unique similarity class of quadratic forms on $V$, and we will refer to this as case $\mathbf{O}^{\circ}$. In general, when the similarity class of the form under consideration is understood, we will write $X(V)$ in place of $X(V, \kappa)$. We will also write either $\overline{X(V)}$ or $\operatorname{PX}(V)$ in place of $\overline{X(V, \kappa)}$ (it will be convenient to retain both pieces of notation for the reduction of $X(A)$ modulo scalars). We will also omit reference to the vector space $V$ when $V$ is understood. The groups $X=X(V)$ and $\bar{X}=\overline{X(V)}=\operatorname{PX}(V)$ are called the classical groups.

Apart from a few cases, the group $\bar{\Omega}$ is simple. The following is [31, Theorem 2.1.3].

THEOREM 6.1. Suppose that $n=\operatorname{dim}_{\mathbb{F}}(V)$ is at least $2,3,4$ and 7 in cases $\mathbf{L}, \mathbf{U}$, $\mathbf{S}$ and $\mathbf{O}$, respectively. Then the group $\bar{\Omega}$ is simple, except for $\mathrm{L}_{2}(2), \mathrm{L}_{2}(3), \mathrm{U}_{3}(2)$ and $\mathrm{PSp}_{4}(2)$.

We also record the following information about $\operatorname{Aut}(\bar{\Omega})$. See [31, Theorem 2.1.4] and the discussion following.

THEOREM 6.2 [31, Theorem 2.1.4 and the discussion following]. Assume that $\bar{\Omega}$ is simple.

(i) If $\bar{\Omega} \neq \mathrm{Sp}_{4}(q)$ with $q$ even, and $\bar{\Omega} \neq \mathrm{P} \Omega_{8}^{+}(q)$, then $\bar{\Sigma}=\operatorname{Aut}(\bar{\Omega})$.

(ii) When $\bar{\Omega}=\mathrm{P} \Omega_{8}^{+}(q)$, we have $[\operatorname{Aut}(\bar{\Omega}): \bar{\Sigma}]=3$.

(iii) When $\bar{\Omega}=\operatorname{Sp}_{4}(q)$ with $q$ even, we have $[\operatorname{Aut}(\bar{\Omega}): \bar{\Sigma}]=2$.

REMARK 6.3. In (ii) and (iii) of Theorem 6.2, an element of Aut $(\bar{\Omega}) \backslash \bar{\Sigma}$ is said to be an exceptional automorphism of $\bar{\Omega}$.

The proof of the following is an easy consequence of the analysis of the structure of outer automorphism groups of classical simple groups in [31, Ch. 2].

LEMMA 6.4. Let $\bar{\Omega}, H$ and $A$ be as above. Assume that we are in case $\boldsymbol{T}$, where $\boldsymbol{T} \in\left\{\mathbf{L}, \mathbf{U}, \mathbf{S}, \mathbf{O}^{\epsilon}\right\}$.

(i) If $A$ is nonabelian, or if $\operatorname{dim}_{\operatorname{End}_{\bar{H}}(A)} V>1$, then $\delta_{\bar{H}}(A)=\delta_{H_{\bar{\Omega}}}(A)$.

(ii) If $A$ is abelian but noncentral, then $\delta_{\bar{H}}(A) \leqslant \delta_{H_{\bar{\Omega}}}(A)+f_{T}(A)$, where $f_{\boldsymbol{T}}(A)=0$ if $\boldsymbol{T} \in\left\{\mathbf{S}, \mathbf{O}^{\epsilon}\right\}$ and $f_{\boldsymbol{T}}(A) \in\{0,1\}$ if $\boldsymbol{T} \in\{\mathbf{L}, \mathbf{U}\}$. 
(iii) If $A$ is central then $\delta_{\bar{H}}(A) \leqslant \delta_{H_{\bar{\Omega}}}(A)+2$, unless $|A|=2$ and $H / H_{\bar{\Omega}}$ has an elementary abelian factor group of order $2^{3}$, in which case $\delta_{\bar{H}}(A)=$ $\delta_{H_{\bar{\Omega}}}(A)+3$.

Proof. Note first that $\bar{H} / H_{\bar{\Omega}}$ is isomorphic to a subgroup of the soluble group $\operatorname{Out}(\bar{\Omega})$. Part (i) then immediately follows.

We now prove (ii) and (iii). By Lemma 3.4 parts (i) and (ii), we have

$$
\delta_{\bar{H}}(A)=\delta_{H_{\bar{\Omega}}}(A)+\delta_{\bar{H} / H_{\bar{\Omega}}}(A) .
$$

Suppose first that $\bar{\Omega}=\mathrm{P} \Omega_{8}^{+}(q)$ so that $\operatorname{Out}(\bar{\Omega}) \cong Z_{f} \times \operatorname{Sym}_{e}$ for some $f \geqslant 1$, where $e=3$ if $q$ is even and $e=4$ if $q$ is odd. Then $\delta_{\bar{H} / H_{\bar{\Omega}}}(A) \leqslant 3$ if $A$ is central, with equality if and only if $f$ is even, $q$ is odd, the image of $\bar{H} / H_{\bar{\Omega}}$ in $\operatorname{Sym}_{e}$ is either a Klein 4-group or $D_{8}$ and the abelianization of $\bar{H} / H_{\bar{\Omega}}$ has an elementary factor group of order $2^{3}$. If $A$ is noncentral and non-Frattini, then we must have that $|A|=3$ or $|A|=2^{2}$ (coming from the Klein 4-subgroup of $\mathrm{Sym}_{4}$ ) and $\delta_{\bar{H} / H_{\bar{\Omega}}}(A) \leqslant 1$.

Thus, we may assume that $\bar{\Omega} \neq \mathrm{P} \Omega_{8}^{+}(q)$. Then $\operatorname{Out}(\bar{\Omega})$ has shape $(D . F) . E$, where $D, F$ and $E$ are cyclic groups and $\operatorname{Out}(\bar{\Omega}) / D$ is abelian. Furthermore, at most two of $D, F$ and $E$ have order greater than 2. Part (iii) then also follows.

Finally, we prove (ii). Since $\operatorname{Out}(\bar{\Omega}) / \operatorname{Frat}(\operatorname{Out}(\bar{\Omega}))$ is abelian in cases $\mathbf{S}$ and $\mathbf{O}^{\epsilon}((n, \epsilon) \neq(8,+))$, part (ii) follows immediately in these cases. So we may assume that we are in cases $\mathbf{L}$ or $\mathbf{U}$. Since $A$ is noncentral, we must have $|A|>2$ and $n \geqslant 3$. Also, $\operatorname{Out}(\bar{\Omega}) / D$ is abelian. Thus, we must have that all noncentral chief factors of $\bar{H} / H_{\bar{\Omega}}$ occur as sections of $D$. Since $D$ is cyclic, it follows from Lemma 3.4 part (iii) that $\delta_{\bar{H} / H_{\bar{\Omega}}}(A) \leqslant 1$. This completes the proof.

We now proceed to the proof of Theorem 2.7 in the classical cases. Throughout, let $G_{0}=\bar{\Omega}$ be a simple classical group as above and let $G$ be group with $G_{0} \leqslant$ $G \leqslant \operatorname{Aut}\left(G_{0}\right)$. For a subgroup $K$ of $\Sigma$ and a symbol $X \in\{\Omega, S, I, \Delta, \Gamma, \Sigma\}$, we will write $K_{X}$ (respectively $K_{\bar{X}}$ ) to denote the group $K \cap X$ (respectively $\overline{K \cap X}$ ).

Throughout, we will write $\hat{G}$ for the unique subgroup of $\Sigma$ containing $Z$ such that $\overline{\hat{G}}=G$. Note that all maximal subgroups of $G$ can be lifted to maximal subgroups of $\hat{G}$. Thus, fixing a maximal subgroup $H$ of $\hat{G}$ and a non-Frattini chief factor $A$ of $\bar{H}$, we need to prove that

(1) $\delta_{\bar{H}}(A) \leqslant 2$ if $A$ is nonabelian.

(2) $\delta_{\bar{H}}(A) \leqslant 2$ if $A$ is abelian but noncentral.

(3) $\delta_{\bar{H}}(A) \leqslant 3$ if $A$ is central, unless $|A|=2$ and $(G, \bar{H})$ is one of the pairs in 
part (ii)(b) of Theorem 1. In this latter case, we have $\delta_{\bar{H}}(A)=d$, where $d$ is as in the last column of Table 1.

Now, we have normal series

$$
\begin{gathered}
H_{\Omega} \leqslant H_{S} \leqslant H_{I} \leqslant H_{\Delta} \leqslant H_{\Gamma} \leqslant H_{\Sigma \cdot a}=H \\
H_{\bar{\Omega}} \leqslant H_{\bar{S}} \leqslant H_{\bar{I}} \leqslant H_{\bar{\Delta}} \leqslant H_{\bar{\Gamma}} \leqslant H_{\overline{\operatorname{Aut}\left(G_{0}\right)}}=\bar{H} .
\end{gathered}
$$

Recall that for a group $G$ and a section $N / M$ of $G$, we write $\delta_{G, N / M}(A)$ for the number of non-Frattini chief factors of $G$ which are $G$-equivalent to $A$ and appear as a section of $N / M$. Using (6.3) and (6.4), we have

$$
\begin{aligned}
\delta_{\bar{H}}(A)= & \delta_{\bar{H}, H_{\bar{\Omega}}}(A)+\delta_{\bar{H}, H_{\bar{S}} / H_{\bar{\Omega}}}(A)+\delta_{\bar{H}, H_{\bar{I}} / H_{\bar{S}}}(A)+\delta_{\bar{H}, H_{\bar{\Delta}} / H_{\bar{I}}}(A)+\delta_{\bar{H}, H_{\bar{\Gamma}} / H_{\bar{\Delta}}}(A) \\
& +\delta_{\bar{H}, \bar{H} / H_{\bar{\Gamma}}}(A)
\end{aligned}
$$

by Lemma 3.4 part (i).

To prove Theorem 2.7 in the classical cases, our strategy will be to analyse the structure of $\bar{H}$ using [9] and part (I) of the main theorem in [31]. We will then apply the expression for $\delta_{\bar{H}}(A)$ in (6.5).

It will be convenient to postpone the cases $\bar{\Omega} \in\left\{\mathrm{Sp}_{4}\left(2^{f}\right), \mathrm{P} \Omega_{8}^{+}(q)\right\}$ and $G \nless \bar{\Gamma}$ until Section 7. Thus, for the remainder of this section, we assume that

$$
\text { if } \bar{\Omega} \notin\left\{\mathrm{Sp}_{4}\left(2^{f}\right), \mathrm{P} \Omega_{8}^{+}(q)\right\} \text { then } G \leqslant \bar{\Gamma} \text {. }
$$

Then by the main theorem of [1], either

(1) $\bar{H}$ is almost simple or

(2) $\bar{H}$ lies in one of eight natural classes $\mathcal{C}_{i}$ of subgroups of $G$.

This was proved, and the eight classes $\mathcal{C}_{i}$, for $1 \leqslant i \leqslant 8$, were defined in [1]. In this paper, we use the definitions of $\mathcal{C}_{i}$ from [31]. If $\bar{H}$ is almost simple, then Theorem 2.7 follows immediately from Proposition 3.12, so we will assume that $\bar{H}$ lies in one of the classes $\mathcal{C}_{i}$ for $1 \leqslant i \leqslant 8$.

We subdivide our proof accordingly. Our first two propositions concern $\mathcal{C}_{1}$. We remark that nonparabolic members of $\mathcal{C}_{1}$ are referred to as subsystem subgroups in [26, Section 2.6], which we reference in the following proof.

Proposition 6.5. Suppose that $H$ lies in class $\mathcal{C}_{1}$ and that $H$ is nonparabolic. Then Theorem 2.7 holds.

Proof. Suppose first that we are in case $\mathbf{O}^{ \pm}$with $q$ and $n$ even and $H$ of type $\operatorname{Sp}_{n-2}(q)$ (see [31, Table 4.1.A]). Then $H_{\bar{\Omega}}$ is nonabelian simple and $\bar{H} / H_{\bar{\Omega}}$ is 
soluble, so $\delta_{\bar{H}}(A)=1$ if $A$ is nonabelian, by Lemma 6.4 part (i). Also, if $A$ is abelian, then $\delta_{\bar{H}}(A)=\delta_{\bar{H}, \bar{H} / H_{\bar{\Omega}}}(A)=f_{\mathbf{O}^{ \pm}}(A)$ in the language of Lemma 6.4, and the result follows.

So we may assume that we are not in case $\mathbf{O}^{ \pm}$with $H$ of type $\operatorname{Sp}_{n-2}(q)$. Then $H$ stabilizes a direct sum decomposition $V=V_{1} \oplus V_{2}$, where each $V_{i}$ is nondegenerate if we are not in case $\mathbf{L}$. Let $\kappa_{i}=\kappa \downarrow_{V_{i}}$, where $\kappa$ is the defining form on $V$. Then, if we are not in case $\mathbf{O}$, we have $n_{1}:=\operatorname{dim} V_{1} \neq n_{2}:=\operatorname{dim} V_{2}$. If we are in case $\mathbf{O}$, then $\kappa_{i}$ is a nondegenerate quadratic form of Witt index $\epsilon_{i} \in\{0$, $\pm 1\}$ on $V_{i}$, and $\left(n_{1}, \epsilon_{1}\right) \neq\left(n_{2}, \epsilon_{2}\right)$. Write $N_{X}\left(V_{1}, V_{2}\right)$ for the full stabilizer of $V_{1} \oplus V_{2}$ in $X$, as $X$ ranges over the symbols $\Omega, S, I$ and $\Delta$. Then we have $N_{\Omega}\left(V_{1}\right.$, $\left.V_{2}\right)=H_{\Omega} \leqslant H_{I} \leqslant N_{I}\left(V_{1}, V_{2}\right)$.

Let $I_{i}$ denote the group of $\kappa_{i}$-isometries of $V_{i}$. Similarly, define $\Omega_{i}$ and $\Delta_{i}$. By [31, Lemma 4.1.1], we have $N_{I}\left(V_{1}, V_{2}\right)=I_{1} \times I_{2}$, and $\Omega_{1} \times \Omega_{2} \leqslant N_{\Omega}\left(V_{1}\right.$, $\left.V_{2}\right)=H_{\Omega}$. Then $L:=\Omega_{1} \times \Omega_{2}$ is characteristic in $H_{I}$, so $L$ is normal in $H$. Hence, we have a normal series

$$
1<\bar{L} \leqslant \bar{H}
$$

Thus, by Lemma 3.4 parts (i) and (ii), we have

$$
\delta_{\bar{H}}(A)=\delta_{\bar{H}, \bar{L}}(A)+\delta_{\bar{H} / \bar{L}}(A)
$$

We now proceed to bound each of the quantities on the right-hand side of (6.7). We first consider the group $\bar{L} \cong \overline{\Omega_{1}} \circ \overline{\Omega_{2}}$. By [31, Lemma 4.1.1], each factor $\overline{\Omega_{i}}$ is normal in $\bar{H}$ and $H_{\bar{I}}$ induces diagonal automorphisms on each $\overline{\Omega_{i}}$ (in fact, it induces the full group of diagonal automorphisms on $\overline{\Omega_{i}}$ ). Furthermore, it is clear that a field automorphism of $G_{0}$ acts as a field automorphism on each factor $\overline{\Omega_{i}}$ in $L$.

Now, assume that a central chief factor of $\bar{H}$ appears in one of the groups $\overline{\Omega_{i}}$. The possibilities for the nonsimple groups appearing as a factor $\overline{\Omega_{i}}$ are listed in [31, Proposition 2.9.2]. It is quickly checked that either $\overline{\Omega_{i}}=\operatorname{Sp}_{4}(2)$ for some $i$ or a nontrivial field or diagonal automorphism of such a group acts nontrivially on all central chief factors appearing in $\overline{\Omega_{i}}$. For example, if $\overline{\Omega_{i}}=\mathrm{P} \Omega_{4}^{+}(3)$, then $\overline{\Omega_{1}}$ has a central chief factor $B$ of order $3^{2}$, but any nontrivial outer automorphism of $G_{0}$ acts nontrivially on $B$. If $q=3$, then any nontrivial diagonal automorphism permutes these two chief factors of order 3 transitively. Whence, a central chief factor of $\bar{H}$ appearing in one of the groups $\overline{\Omega_{i}}$ implies that either $\bar{H} / H_{\bar{\Omega}} \cong$ $G / G_{0} \leqslant \operatorname{Out}\left(G_{0}\right)$ intersects both the group of diagonal and field automorphisms of $G_{0}$ trivially or $q=2$ and $\left|G / G_{0}\right| \leqslant 2$. Thus, $\left|\bar{H} / H_{\bar{\Omega}}\right| \leqslant 2$, with equality possible only in case $\mathbf{L}$ or case $\mathbf{S}$ with $q$ even. Since $\overline{\Omega_{i}}$ is either simple or is one of the groups appearing in [31, Proposition 2.9.2] (each of which has $\delta_{\overline{\Omega_{i}}}(B) \leqslant 2$ 
for all non-Frattini chief factors $B$, with equality possible only if $B$ is central and $\overline{\Omega_{i}} \in\left\{\mathrm{U}_{3}(2), \mathrm{P} \Omega_{4}^{+}(3)\right\}$ ), we deduce that either $\delta_{\bar{H}}(A) \leqslant 3$ (respectively 1 ) if $A$ is central (respectively noncentral) or all non-Frattini chief factors of $\bar{H}$ contained in $\bar{L}$ are noncentral (only one of the exceptional groups $\overline{\Omega_{i}} \in\left\{\mathrm{U}_{3}(2), \mathrm{P} \Omega_{4}^{+}(3)\right\}$ can occur, and in that case, $G / G_{0}$ must be trivial by the arguments above). Thus, for the remainder of the proof, we may assume the following.

All non-Frattini chief factors of $\bar{H}$ contained in $\bar{L}$ are noncentral.

Now, if $\overline{\Omega_{1}}$ and $\overline{\Omega_{2}}$ are nonabelian simple, then $\delta_{\bar{H}, \bar{L}}(A) \leqslant 1$ if $A$ is nonabelian since $\overline{\Omega_{1}}$ and $\overline{\Omega_{2}}$ have distinct centralizers in $\bar{H}$. Also, $\delta_{\bar{H}, \bar{L}}(A)=0$ if $A$ is abelian by Lemma 3.5. Next, assume that $\overline{\Omega_{1}}$ is nonsimple. Then the possibilities for $\overline{\Omega_{1}}$ are listed in [31, Proposition 2.9.2]. We can then see that $\delta_{\overline{\Omega_{1}}}(A) \leqslant 1$ since we are assuming that $A$ is noncentral. It follows that

$$
\delta_{\bar{H}, \bar{L}}(A) \leqslant 1
$$

with equality only if $A$ is noncentral.

Next, we consider the group $Y:=H_{\bar{I}} / \bar{L}$. If $n_{1}=1$ in case $\mathbf{O}$, then $\bar{H}$ is almost simple with socle $\overline{\Omega_{2}}$ by [31, Proposition 4.1.6]. The required bounds then follow immediately from Lemma 6.4. So we may assume that $n_{i}>1$ if we are in case O. Then by [31, Lemma 4.1.1], we have that $Y$ is a subdirect product of the group $\left(\overline{I_{1}} / \overline{\Omega_{1}}\right) \times\left(\overline{I_{2}} / \overline{\Omega_{2}}\right)$.

Now, by checking the nonsimple groups $\overline{\Omega_{i}}$ appearing in [31, Proposition 2.9.2], we can see that no chief factor of $\overline{I_{i}}$ can appear in both $\overline{I_{i}} / \overline{\Omega_{i}}$ and $\overline{\Omega_{i}}$. Thus, since $H / H_{\bar{I}} \leqslant \operatorname{Aut}\left(G_{0}\right) / I$ is abelian, we deduce from (6.9) and Lemma 3.4 that $\delta_{\bar{H}}(A)=\delta_{\bar{H}, H_{\bar{T}}}(A) \leqslant 2$ if $A$ is noncentral. Since $\overline{\Omega_{1}}$ and $\overline{\Omega_{2}}$ have distinct centralizers in $\bar{H}$ in the case when one of them is nonabelian simple, we also have that $\delta_{\bar{H}}(A) \leqslant 1$ if $A$ is nonabelian.

Thus, we may assume for the remainder of the proof that $A$ is central. In particular,

$$
\delta_{\bar{H}}(A)=\delta_{\bar{H} / \bar{L}}(A)
$$

by (6.8).

Suppose first that we are not in case $\mathbf{O}$ so that $\Delta=I$. If we are in case $\mathbf{S}$ or case $\mathbf{U}$, then $\bar{H} / H_{\bar{I}}$ is cyclic. If $|A|$ is odd, then $|A|$ is prime (since $A$ is central) and the $|A|$-part of $\bar{H} / H_{\bar{I}}$ is cyclic. The result then follows immediately from Lemma 3.4 and (6.10) since $H_{\bar{I}} / \bar{L}$ is metacyclic. So we may assume that we are in case $\mathbf{L}$ and that $|A|=2$. Then from the embedding of the group $H$ into $\mathrm{GL}_{n}(q)$, we can see that $Y_{1}:=Y \cap\left(H_{\bar{\Omega}} / \bar{L}\right)$ is a subgroup of $\left\{\left(\alpha, \alpha^{-1}\right): \alpha \in \mathbb{F}_{q}^{\times}\right\} \leqslant \mathbb{F}_{q}^{\times} \times \mathbb{F}_{q}^{\times}$. Now, if $n_{i}$ is odd, then $\left|\overline{I_{i}} / \overline{\Omega_{i}}\right|$ is odd, so the bound $\delta_{\bar{H} / \bar{L}}(A) \leqslant 3$ follows as above. So we may 
assume further that each $n_{i}$ is even. Then $\left|Y_{1}\right|$ is even, by [31, Proposition 4.1.4]. It is now clear that if $G / G_{0}=\bar{H} / H_{\bar{\Omega}}$ intersects $\bar{I} / \bar{\Omega}$ in a group of even order, then the $\bar{H}$-module $Y /\left(Y^{2} \operatorname{Frat}(Y)\right)$ has order $2^{2}$ (this is because the 2-part of $Y$ is clearly noncyclic in this case). In fact, from the relations on $\operatorname{Out}\left(G_{0}\right)$ given in Section 1, we have that $Y /\left(Y^{2} \operatorname{Frat}(Y)\right)$ is a trivial $\bar{H}$-module (recall that the field automorphisms on $G_{0}$ induce field automorphisms on each $\overline{\Omega_{i}}$ ). The module $Y /\left(Y^{2} \operatorname{Frat}(Y)\right)$ is also non-Frattini in the associated quotient $\bar{H} / \bar{L}$. One can see this by observing that each generator reduces modulo $H_{\bar{\Omega}}$ to an element of an irredundant generating set for $G / G_{0}$ (again, see the generators and relations for $\operatorname{Out}\left(G_{0}\right)$ in Section 1). Whence, we get

$$
\delta_{\bar{H}}(A)=\delta_{\bar{H} / \bar{L}}(A)=1+c+e+h,
$$

where $c:=1$ if the intersection of $G / G_{0}$ with $\bar{I} / \bar{\Omega}$ has even order, and $c:=0$ otherwise; $e:=1$ if the intersection of $G / G_{0}$ with the group of field automorphisms of $G_{0}$ has even order, and $e:=0$ otherwise; and $h:=1$ if $G$ contains a graph automorphism of $G_{0}$, and $h:=0$ otherwise. This gives us what we need.

Finally, assume that we are in case $\mathbf{O}$. The bound $\delta_{\bar{H}}(A) \leqslant 3$ clearly holds in this case if $|A|$ is odd, so we will assume for the remainder of the proof that $|A|=2$. Then arguing as in the paragraph above, we get that

$$
\delta_{\bar{H}}(A)=\delta_{\bar{H}}(Y)+d(K),
$$

where $K:=\left(G / G_{0}\right) \cap\left(\operatorname{Aut}\left(G_{0}\right) / \bar{I}\right)$. This reduces our problem to examining the group $Y$. We do this using [31, Proposition 4.1.6].

First, if both $n_{1}$ and $n_{2}$ are odd, or if $q$ is even, then $Y \leqslant Z_{2} \times Z_{2}$ and $K$ is cyclic, so the bound $\delta_{\bar{H}}(A) \leqslant 3$ follows from (6.11). So we may assume that $n_{2}$ is even and that $q$ is odd. If $n_{1}$ is odd, then $n$ is odd and $Y$ is isomorphic to a subgroup of $Z_{2} \times 2^{2}$. Also, $Y_{1} \leqslant Y$ has shape $\operatorname{diag}\left(Z_{2} \times Z_{2}\right) .2 \leqslant Z_{2} \times 2^{2}$ by [31, proof of Proposition 4.1.6]. If $G / G_{0}$ intersects $\bar{\Delta} / \bar{\Omega}$ nontrivially, then $H_{\bar{\Delta}} /\left(C_{\bar{H}}\left(\Omega_{2}\right) \overline{\Omega_{2}}\right) \cong D_{8}$, so one of the $Z_{2}$ factors in the group $Y$ is Frattini. Thus, $\delta_{H}(Y) \leqslant 2$. Otherwise, $G / G_{0}$ contains no diagonal automorphisms, so $Y=Y_{1}$ and we also get $\delta_{Y}(A)=2$. Either way, we get $\delta_{\bar{H}}(A) \leqslant 3$ by (6.11).

Thus, we may assume $q$ is odd and both $n_{1}$ and $n_{2}$ are even. Let $s_{\epsilon_{i}}:=2$ if $\epsilon_{i}=+$ and $s_{\epsilon_{i}}:=1$ otherwise. If the discriminants of both $\kappa_{i}$ are nonsquare, then $\kappa$ has square discriminant (recall that $q$ is odd). Also, $\left|H_{\bar{\Omega}} / \bar{L}\right|=2$, by [31, Proposition 4.1.6]. We then get that $H_{\bar{\Delta}} / \bar{L}$ is isomorphic to a subgroup of a group of shape $2^{s_{\epsilon_{1}}} \times 2^{s_{\epsilon_{2}}}$, with $\left|H_{\bar{\Omega}} / \bar{L}\right|=2$ and $H_{\bar{\Delta}} / H_{\bar{\Omega}}$ is precisely the intersection of $G / G_{0}$ with $\bar{\Delta} / \bar{\Omega} \leqslant D_{8}$. We then immediately deduce from Lemma 3.4 that

$$
\delta_{\bar{H}}(A)=\delta_{\bar{H} / \bar{L}}(A)=\delta_{\bar{H}, H_{\bar{\Delta}} / \bar{L}}(A)+\delta_{\left|G / G_{0} \cap \bar{\Gamma} / \bar{\Delta}\right|, 2} \leqslant 4,
$$


with equality if and only if $\left(G / G_{0}\right) \cap(\Gamma / \Delta)$ is even and $G / G_{0}$ intersects the group of diagonal automorphisms on $G_{0}$ in an elementary abelian subgroup of order $2^{2}$, which is true if and only if (6.8) holds. Note that (6.8) can hold only if $q$ is odd and the $\epsilon_{i}$ are not both -, since the intersection of the group of diagonal automorphisms of $G_{0}$ with $G / G_{0}$ has order at most 2 in these cases. This gives the first $\mathcal{C}_{1}$ case in Table 1 for case $\mathbf{O}$.

Assume next that the discriminant of $\kappa_{2}$ is a square. Then $H_{\bar{\Delta}} / H_{\bar{\Omega}}$ is isomorphic to a subgroup of a group of shape $D_{8} \times D_{8}$, and $H_{\bar{\Omega}} / L \leqslant 2^{2} \times 2^{2} \leqslant H_{\bar{\Delta}}$ has order 4, by [31, Proposition 4.1.6]. If the discriminant of $\kappa$ is a nonsquare, then the discriminant of $\kappa_{1}$ is a nonsquare and we have $H_{\bar{I}} \leqslant 2^{s_{\epsilon_{1}}} \times 2^{2}$ and $H_{\bar{\Delta}} / \bar{L}$ is an elementary abelian 2-group. Hence,

$$
\begin{aligned}
\delta_{\bar{H}}(A) & =\delta_{\bar{H}, \bar{H} / \bar{L}}(A)=\delta_{\bar{H}, H_{\bar{\Delta}} / \bar{L}}(Y)=\delta_{\bar{H}, H_{\bar{\Omega}} / \bar{L}}(A)+\delta_{\bar{H}, H_{\bar{\Delta}} / H_{\bar{\Omega}}}(A)+\delta_{\bar{H} / H_{\bar{\Delta}}}(A) \\
& =2+d\left(G / G_{0}\right) .
\end{aligned}
$$

If the discriminant of $\kappa$ is a square, then either $\left(G / G_{0}\right) \cap(\bar{\Delta} / \bar{\Omega})$ equals $D_{8}$ and we get $\delta_{\bar{H}, H_{\bar{\Delta}} / \bar{L}}(A)=3$ (precisely one of the $Z_{2}$-factors in $H_{\bar{\Omega}} / \bar{L}$ becomes Frattini in this case) or $\left(G / G_{0}\right) \cap(\bar{\Delta} / \bar{\Omega})$ does not equal $D_{8}$. In this latter case, we get that $H_{\bar{\Delta}} / \bar{L} \leqslant 2^{2} \times 2^{2}$ is elementary abelian, so as above, we get $\delta_{\bar{H}}(A)=2+d\left(G / G_{0}\right)$. These cases combine to give the second $\mathcal{C}_{1}$ case in Table 1 for case $\mathbf{O}$, and this completes the proof.

Proposition 6.6. Suppose that $H \in \mathcal{C}_{1}$ is a parabolic subgroup. Then Theorem 2.7 holds.

Proof. In this case, either

(i) $H$ stabilizes a totally singular subspace $W \subset V$ of dimension $m \leqslant n / 2$ or

(ii) we are in case $\mathbf{L}, G$ contains a graph or graph-field automorphism and $H$ stabilizes subspaces $W<U$ with $\operatorname{dim} W=m<n / 2$, and $\operatorname{dim} U=n-m$.

We will say that $H$ is of type $P_{m}$ in case (i) and that $H$ is of type $P_{m, n-m}$ in case (ii).

Suppose first that we are in case (i), and write $N_{X}(W)$ for the full stabilizer of $W$ in $X$, as $X$ ranges over the symbols $\Omega, S, I$ and $\Delta$. Similarly, define the centralizers $C_{X}(W) \leqslant N_{X}(W)$. Hence, we have $N_{\Omega}(W)=H_{\Omega} \leqslant H_{I} \leqslant N_{I}(W)$.

By [31, Lemmas 4.1.12 and 4.1.13], there exist subspaces $U$ and $Y$ of $V$, with $Y$ totally singular, such that $V=(W \oplus Y) \perp U(U=0$ in case $L)$ and $N_{I}(W)=$ $C \rtimes L$, where

$$
C:=C_{I}(W) \cap C_{I}\left(W^{\perp} / W\right) \cap C_{I}\left(V / W^{\perp}\right) \quad \text { and } \quad L:=N_{I}(W) \cap N_{I}(Y) \cap N_{I}(U) .
$$


Moreover, $\operatorname{dim} Y=m$ unless we are in case $\mathbf{L}$, in which case $\operatorname{dim} Y=n-m$. The group $C$ is called the unipotent radical of $H$, while $L$ is called a Levi subgroup of $H$. It is an easy exercise to show that $C$ is a nilpotent characteristic subgroup of $H_{\Omega}$, that $C / C^{\prime}$ is an elementary abelian $p$-group and that $C$ is contained in $N_{\Omega}(W)$. In particular, $C^{\prime} \leqslant \operatorname{Frat}(C)$. Furthermore, by [5, Theorem 2], the $L$-module $C / C^{\prime}$ is either irreducible or has composition length 2 , with nonisomorphic $L$-composition factors. It follows from Clifford's theorem that $C / C^{\prime}$ is an $\mathbb{F}_{p}[\bar{H}]$-module, with composition length at most 2 , and if it has composition length 2 , then the factors are nonisomorphic.

We now consider the structure of $L$. Let $I(U)$ denote the group of $\kappa$-isometries of the $(n-2 m)$-dimensional vector space $U$. Similarly, define $\Omega(U)$. Now, recall that $u:=2$ if we are in case $\mathbf{U}$, and $u:=1$ otherwise. Then in cases $\mathbf{U}, \mathbf{S}$ and $\mathbf{O}$, we have, by [31, Lemma 4.1.12], that $L \cong \mathrm{GL}_{m}\left(q^{u}\right) \times I(U)$. In case $\mathbf{L}$, we have, by [31, Lemma 4.1.1], that $L \cong \mathrm{GL}_{m}(q) \times \mathrm{GL}_{n-m}(q)$. Let $M:=\mathrm{SL}_{m}(q) \times$ $\mathrm{SL}_{n-m}(q) \unlhd L$ in case $\mathbf{L}$, and let $M$ be the normal subgroup $\mathrm{SL}_{m}\left(q^{u}\right) \times \Omega(U)$ of $L$ otherwise. Then $M \leqslant N_{\Omega}(W) \leqslant L_{\Omega} \leqslant H_{\Omega}$. Furthermore, since $C \rtimes M$ is characteristic in $C \rtimes L \unlhd H$, it is normal in $H$. Hence, $C \rtimes M$ is normal in $H$. Thus, we have a normal series

$$
1<\bar{C} \leqslant \bar{C} \rtimes \bar{M} \leqslant \bar{C} \rtimes L_{\bar{\Omega}}=H_{\bar{\Omega}} \leqslant \bar{H}
$$

for $\bar{H}$. Thus, by Lemma 3.4, we have

$$
\delta_{\bar{H}}(A)=\delta_{\bar{H}, \bar{C}}(A)+\delta_{\bar{H}, \bar{M}}(A)+\delta_{\bar{H}, L_{\bar{\Omega}} / \bar{M}}(A)+\delta_{\bar{H}, \bar{H} / H_{\bar{\Omega}}}(A) .
$$

As in the proof of Proposition 6.5, we now proceed to bound each of the quantities on the right-hand side of (6.13).

First, as remarked above, the $\mathbb{F}_{p}[\bar{H}]$-module $C / C^{\prime}$ is either irreducible or has composition length 2 , with nonisomorphic $H$-composition factors. In particular, $\delta_{\bar{H}, C}(A) \leqslant 1$, and $\delta_{\bar{H}, \bar{C}}(A)=0$ if $A$ is not a $p$-group.

Now, from (6.12), $\bar{H} / \bar{C}$ has a normal series identical to the normal series in (6.6) in the proof of Proposition 6.5. From this proof, one quickly sees that no chief factor of $\bar{H} / \bar{C}$ can have dimension greater than 2 . Thus, by the paragraph above, we have $\delta_{\bar{H}}(A)=1$ if $A$ is contained in $C$. Also, for any non-Frattini chief factor $A \neq C / C^{\prime}$ of $\bar{H}$, we have $\delta_{\bar{H}}(A)=\delta_{\bar{H} / \bar{C}}(A)$.

If $H$ is of type $P_{m}$, then the arguments in cases $\mathbf{U}$ and $\mathbf{S}$ are almost identical to the arguments in the proofs of these cases in Proposition 6.5. That is, we get $\delta_{\bar{H}}(A)=\delta_{\bar{H} / \bar{C}}(A) \leqslant 2$ (respectively 3 ) if $A$ is noncentral (respectively central). If $H$ is of type $P_{m}$ in case $\mathbf{L}$, then $\bar{H}$, and hence $G$, cannot contain an element of $\operatorname{Aut}\left(G_{0}\right)-\bar{\Gamma}$ since such an element interchanges the maximal subgroups $P_{m}$ and $P_{n-m}$ of $\bar{\Omega}$. Thus, we also have $\delta_{\bar{H}}(A)=\delta_{\bar{H} / \bar{C}}(A) \leqslant 2$ (respectively 3 ) if $A$ is noncentral (respectively central) in this case. 
So we may assume that we are either in case $\mathbf{L}$ with $H$ of type $P_{m, n-m}$ or we are in case $\mathbf{O}$. Suppose first that we are in case $\mathbf{L}$ with $H$ of type $P_{m, n-m}$. We now argue exactly as in the $\mathbf{L}$ case of Proposition 6.5. The only slight difference is that the group $Y:=H_{\bar{\Omega}} / \bar{L}$ is a subgroup of $\left\{\left(\alpha, \beta, \alpha^{-1}, \beta^{-1}\right): \alpha, \beta \in \mathbb{F}_{q}^{\times}\right\} \leqslant \mathbb{F}_{q}^{\times} \times \mathbb{F}_{q}^{\times}$. We then get that $Y=H_{\bar{\Omega}} / \bar{L}$ has even order if and only if $q$ is odd and both $m$ and $n-m$ are even. It then follows that if $G / G_{0}=\bar{H} / H_{\bar{\Omega}}$ intersects $\bar{I} / \bar{\Omega}$ in a group of even order, then the $\bar{H}$-module $Y /\left(Y^{2} \operatorname{Frat}(Y)\right)$ has order $2^{2}$ (this is again because the 2-part of $Y$ is noncyclic in this case). The relations on $\operatorname{Out}\left(G_{0}\right)$ given in Section 1 then imply that $Y /\left(Y^{2} \operatorname{Frat}(Y)\right)$ is a trivial $\bar{H}$-module (since the field automorphisms on $G_{0}$ induce field automorphisms on each $\overline{\Omega_{i}}$ ). The module $Y /\left(Y^{2} \operatorname{Frat}(Y)\right)$ is also non-Frattini in the associated quotient $\bar{H} / \bar{L}$ as before, and, hence, we get

$$
\delta_{\bar{H}}(A)=\delta_{\bar{H} / \bar{L}}(A)=1+c+e+h,
$$

where $c:=1$ if the intersection of $G / G_{0}$ with $\bar{I} / \bar{\Omega}$ has even order, and $c:=0$ otherwise; $e:=1$ if the intersection of $G / G_{0}$ with the group of field automorphisms of $G_{0}$ has even order, and $e:=0$ otherwise; and $h:=1$ if $G$ contains a graph automorphism of $G_{0}$, and $h:=0$ otherwise. This gives us the second $\mathcal{C}_{1}$ case in Table 1 for groups in case $\mathbf{L}$.

So assume that we are in case $\mathbf{O}$. By [31, Lemma 4.1.12 and its proof], we have $H_{\bar{\Omega}}=K \times \overline{\Omega(U)}$, where either $K$ is a subgroup of $\mathrm{GL}_{m}(q)$ containing $\mathrm{SL}_{m}(q)$ or $K$ is a subgroup of $\mathrm{PGL}_{m}(q)$ containing $\mathrm{L}_{m}(q)$. Also, all non-Frattini chief factors of $\bar{H} / H_{\bar{\Omega}}$ are central, by Lemma 6.4. Since the groups $K$ and $\overline{\Omega(U)}$ are normal and have distinct centralizers in $\bar{H}$, we deduce that if $A$ is noncentral, then $\delta_{\bar{H}}(A)=\delta_{\overline{\Omega(U)}}(A)$ or $\delta_{\bar{H}}(A)=\delta_{K}(A)$. By examining the non-almost-simple groups which can occur in $H_{\bar{\Omega}}$ (from [31, Proposition 2.9.2]), we can see that $\delta_{E}(B) \leqslant 1$ for all non-Frattini chief factors $B$ of $E$, for each $E \in\{K, \overline{\Omega(U)}\}$. Hence, we have $\delta_{\bar{H}}(A) \leqslant 1$ if $A$ is noncentral, and this gives us what we need. In fact, since $\bar{H} / H_{\bar{\Omega}} \leqslant D_{8} \times Z_{f}$, we can also deduce that $\delta_{\bar{H}}(A) \leqslant 2$ if $A$ is central and has odd order.

So to complete the proof, we may assume that $|A|=2$. Since a field automorphism of $G_{0}$ also acts as a field automorphism on each central factor of $H_{\bar{\Omega}}$, we deduce (arguing as in the proof of Proposition 6.5) that either $G$ contains no field automorphisms or any non-Frattini chief factor of $\bar{H}$ contained in $H_{\bar{\Omega}}$ is noncentral. Suppose first that $G$ contains no field automorphisms. Then $\bar{H} / H_{\bar{\Omega}}$ is isomorphic to a subgroup of $D_{8}$, and so has at most two non-Frattini chief factors $G$-equivalent to $A$. By the argument in the paragraph above, we also have $\delta_{\overline{\Omega(U)}}(A) \leqslant 2$, with equality if and only if $n-m=4, \Omega(U) \cong \Omega_{4}^{+}(3)$, and $G / G_{0}$ is trivial. However, in this case, we get $\delta_{\bar{H} / \bar{C}}(A) \leqslant 3$ using Lemma 3.4 (with the additional $Z_{2}$-factor possibly coming from $K$ ). Thus, we may assume that all chief 
factors of $\bar{H}$ contained in $H_{\bar{\Omega}}$ are noncentral. Then $\delta_{\bar{H}}(A)=\delta_{\bar{H} / H_{\bar{\Omega}}}(A) \leqslant 3$, by Lemmas 3.4 and 6.4.

Thus, we have $\delta_{\bar{H}}(A) \leqslant 3$ in case $\mathbf{O}$ and any type, and this completes the proof.

Proposition 6.7. Suppose that $H$ lies in class $\mathcal{C}_{2}$. Then Theorem 2.7 holds.

Proof. In this case, $H$ is the stabilizer in $G$ of a subspace decomposition

$$
V=V_{1} \oplus V_{2} \oplus \cdots \oplus V_{t}
$$

satisfying the conditions in [31, Table 4.2.A]. In particular, $V_{i}$ is either a nondegenerate $m$-space for all $i$ or a totally singular $m$-space for all $i$ (the dimension $m$ is fixed). Write $\mathcal{D}:=\left\{V_{1}, \ldots, V_{t}\right\}$, and let $I_{i}$ denote the group of $\kappa$ isometries of $V_{i}$. Similarly, define $\Omega_{i}$ and $\Delta_{i}$. Also, write $X_{\mathcal{D}}$ for the full stabilizer of $\mathcal{D}$ in $X$, as $X$ ranges over the symbols $\Omega, S, I, \Delta, \Gamma$ and $\Sigma$. Denote the kernel of the action of $X_{\mathcal{D}}$ on $\mathcal{D}$ by $X_{(\mathcal{D})} \unlhd X_{\mathcal{D}}$ and the induced action of $X_{\mathcal{D}}$ on $\mathcal{D}$ by $X^{\mathcal{D}}$. In particular, $X^{\mathcal{D}} \cong X_{\mathcal{D}} / X_{(\mathcal{D})} \leqslant \operatorname{Sym}_{t}$.

Since $\Omega \leqslant G$, we have $\Omega_{\mathcal{D}} \leqslant G_{\mathcal{D}}=H$. Hence, $\Omega_{\mathcal{D}}=H_{\Omega} \leqslant H_{\Delta} \leqslant \Delta_{\mathcal{D}}$, and we have a normal series

$$
1<H_{\overline{\Omega_{(\mathcal{D})}}} \leqslant H_{\bar{\Omega}} \leqslant \bar{H}
$$

Thus, by Lemma 3.4, we have

$$
\delta_{\bar{H}}(A)=\delta_{\bar{H}, H_{\overline{\Omega_{(\mathcal{D})}}}}(A)+\delta_{\bar{H}, H_{\overline{\Omega^{\mathcal{D}}}}}(A)+\delta_{\bar{H}, \bar{H} / H_{\bar{\Omega}}}(A) .
$$

Now, the members of $\mathcal{C}_{2}$ are distinguished into types in [31, Table 4.2.A], and we divide our proof accordingly.

Suppose first that $H$ is of type $\left.\mathrm{GL}_{m}(q)_{2} \operatorname{Sym}_{t}, \mathrm{GU}_{m}(q)\right)_{2} \operatorname{Sym}_{t}, \operatorname{Sp}_{m}(q)$ 2 $\operatorname{Sym}_{t}$ or $\mathrm{O}_{m}^{\epsilon}(q)$ 2 $\operatorname{Sym}_{t}$. Then the spaces $V_{i}$ are mutually isometric. Moreover, [31, Lemma 4.2.8 part (iii)] implies that

$$
H_{\Omega}=\Omega_{\mathcal{D}}=\Omega_{(\mathcal{D})} J_{\Omega} \leqslant \Delta_{1} 2 \operatorname{Sym}_{t},
$$

where $J_{\Omega}=\operatorname{Sym}_{t}$, apart from case $\mathbf{O}$ with $m=1$ and $q \equiv \pm 3(\bmod 8)($ see $[31$, Proposition 4.2.15]). In this case, $J_{\Omega}=\mathrm{Alt}_{t}$. Either way, we have

(a) $\Delta_{1} / \Omega_{1}$ has abelian Frattini quotient;

(b) $\Omega_{(\mathcal{D})} \geqslant \Omega_{1}^{t}$; and

(c) $H_{\Omega_{(\mathcal{D})}} / \Omega_{1}^{t}$ is the deleted subgroup of $\left(\Delta_{1} / \Omega_{1}\right)^{t}$. 
Finally, since $N_{H}\left(\Delta_{1}\right)$ is precisely the stabilizer in $H$ of $V_{1}$, [31, Lemma 4.2.1] implies that $H=N_{H}\left(\Delta_{1}\right) H_{\Omega}$.

Thus, we can use Lemma 3.9, together with Lemma 3.10, to find the chief factors of $\bar{H}$ contained in $H_{\overline{\Omega_{(\mathcal{D})}}}$. Indeed, note that the outer automorphism group $T$ induced by the action of $N_{\bar{H}}\left(\mathrm{P} \Omega_{1}\right)$ on $\mathrm{P} \Omega_{1}$ is isomorphic to the outer automorphism group induced by $\bar{H}$ on $\bar{\Omega}$. Hence, by Lemma 3.9, apart from case $\mathbf{O}$ with $q$ odd, there is, for each prime $r$, at most one non-Frattini chief factor of $\bar{H}$ contained in $H_{\overline{\Omega_{(\mathcal{D})}}} / \mathrm{P} \Omega_{1}^{t}$ (which, if it exists, is noncentral): in the case $r \mid t$ and $t>2$, there is one non-Frattini chief factor of order $r^{t-2}$; in the case $r \nmid t$, there is one of order $r^{t-1}$; while if $t=r=2$, there are no non-Frattini chief factors of $\bar{H}$ contained in $H_{\overline{\Omega_{(\mathcal{D})}}} / \mathrm{P} \Omega_{1}^{t}$.

Next, assume that we are in case $\mathbf{O}$, with $q$ odd. If $H_{\bar{\Delta}} / H_{\bar{\Omega}} \cong D_{8}$, then $T$ permutes the two chief factors of order 2 of $H_{\bar{I}} / H_{\bar{\Omega}}$. Otherwise, $T$ fixes them. Hence, if $H_{\bar{\Delta}} / H_{\bar{\Omega}}<D_{8}$, then there are two non-Frattini chief factors of $\bar{H}$ contained in $H_{\overline{\Omega_{(\mathcal{D})}}} / \mathrm{P} \Omega_{1}^{t}$ if $t>2$, and none if $t=2$ : in the case $t>2$ is even, both have order $2^{t-2}$. If $t>2$ is odd, both have order $2^{t-1}$. If $H_{\bar{\Delta}} / H_{\bar{\Omega}} \cong D_{8}$, then there is one non-Frattini chief factor of $\bar{H}$ contained in $H_{\overline{\Omega_{(\mathcal{D})}}} / \mathrm{P} \Omega_{1}^{t}$ if $t>2$ and none if $t=2$ : in the case $t>2$ is even, the chief factor has order $2^{2(t-2)}$. If $t>2$ is odd, it has order $2^{2(t-1)}$. In any case, $H_{\overline{\Omega_{(\mathcal{D})}}} / \mathrm{P} \Omega_{1}^{t}$ comprises either one or two noncentral non-Frattini chief factors of $\bar{H}$.

Next, we consider the non-Frattini chief factors of $\bar{H}$ contained in $\mathrm{P} \Omega_{1}^{t}$. If $\mathrm{P} \Omega_{1}$ is simple, then $\mathrm{P} \Omega_{1}^{t}$ is a nonabelian chief factor of $\bar{H}$, by Lemma 3.9. If $\mathrm{P} \Omega_{1}=\mathrm{Sp}_{4}(2)$, then $\mathrm{P} \Omega_{1}^{t}$ comprises three non-Frattini chief factors of $\bar{H}$ : the nonabelian $\mathrm{Alt}_{6}^{t}$; a noncentral abelian factor of order $2^{t-(2, t)}$; a central factor of order 2, again by Lemma 3.9. Finally, if $\mathrm{P} \Omega_{1}$ is nonsimple, then $\mathrm{P} \Omega_{1}$ is listed in [31, Proposition 2.9.2]. In particular, by Lemma 3.10, we have $\delta_{\mathrm{P} \Delta_{1}, \mathrm{P} \Omega_{1}}(B) \leqslant 1$ for any non-Frattini chief factor $B$ of $\mathrm{P} \Delta_{1}$, with equality if and only if $W$ is noncentral and contained in $\mathrm{P} \Delta_{1}$. It follows, again using Lemma 3.9, that for each non-Frattini chief factor $W$ of $\mathrm{P} \Delta_{1}$ contained in $\mathrm{P} \Omega_{1}$, we get a unique nonFrattini chief factor, $\bar{H}$-equivalent to $W^{t}$, contained in $\mathrm{P} \Omega_{1}^{t}$. This is noncentral, again by Lemma 3.9.

In summary, we have $\delta_{\bar{H}, H_{\overline{\Omega_{(\mathcal{D})}}}}(A) \leqslant 1$, with equality possible only if either $A$ is noncentral or $|A|=2$ and $\mathrm{P} \Omega_{1}=\operatorname{Sp}_{4}(2)$.

Thus, we have determined the quantity $\delta_{\bar{H}, H_{\overline{\Omega_{(\mathcal{D})}}}}(A)$. We now determine the other quantities in the bound (6.15). Note that $\bar{H} / H_{\overline{\Omega^{\mathcal{D}}}} \cong J .\left(\bar{H} / H_{\bar{\Omega}}\right)$, and $J=$ Sym $_{t}$, unless we are in case $\mathbf{O}$ with $m=1$ and $q \equiv \pm 3(\bmod 8)$, in which case $J=$ Alt $_{t}$. Suppose that we are not in this latter case. Then the Frattini subgroup of $\bar{H} / H_{\overline{\Omega^{\mathcal{D}}}}$ is contained in the centralizer of the Alt $t_{t}$ normal subgroup since $\operatorname{Out}\left(\mathrm{Alt}_{t}\right)$ is elementary abelian of order 1,2 or $2^{2}$. In particular, the $\bar{H}$-chief factor 
$Z_{2}$ in $J=\operatorname{Sym}_{t}$ is non-Frattini in $\bar{H}$. Thus, we conclude that $\delta_{\bar{H}, H_{\Omega^{\mathcal{D}}}}(A) \leqslant 1$, with equality if and only if either

(a) $|A|=2$ (so that $A$ is central) and we are not in case $\mathbf{O}$ with $m=1$ and $q \equiv \pm 3(\bmod 8)$

(b) $V \cong \mathrm{Alt}_{t}$ and $t \neq 2,4$; or

(c) $t=4$ and $|A| \in\{3,4\}$.

Finally, using Lemma 6.4 , we see that $\delta_{\bar{H}, \bar{H} / H_{\bar{\Omega}}}(A) \leqslant 3$, with equality if and only if $|A|=2$ and $(\star)$ holds (see Page 3 for the definition of $(\star)$ ).

Next, assume that $m=n / 2$ and that $H$ is of type $\mathrm{GL}_{n / 2}\left(q^{u}\right) .2$. Then $H_{I}$ has shape $\operatorname{GL}_{n / 2}\left(q^{u}\right) .2$ by [31, Corollary 4.2 .2 part (ii) and Lemma 4.2.3]. Here, $H_{I} / \mathrm{GL}_{n / 2}\left(q^{u}\right)=H_{I}^{\mathcal{D}} \cong \operatorname{Sym}_{2}$ is the induced action of $H_{I}$ on $\mathcal{D}$. If $n=4$ and $q^{u} \leqslant 3$, then $G$ has no maximal subgroups in class $\mathcal{C}_{2}$ of this form, using the tables in [9, Ch. 8]. So assume that $\operatorname{SL}_{n / 2}\left(q^{u}\right)$ is perfect. Then $Z \leqslant \operatorname{Frat}\left(\operatorname{SL}_{n / 2}\left(q^{u}\right)\right)$ (recall that $Z$ is the scalar subgroup of $I$ ). Hence, since $H_{I} / \operatorname{SL}_{n / 2}\left(q^{u}\right)$ is cyclic, we have $\delta_{H_{\bar{I}}}(A) \leqslant 1$. Since $\bar{H} / H_{\bar{I}}$ is abelian metacyclic, it follows that $\delta_{\bar{H}}(A) \leqslant 3$, with equality possible only if $A$ is central.

Finally, assume that $m=n / 2$ and that $H$ is of type $O_{n / 2}(q)^{2}$ so that we are in case $\mathbf{O}^{ \pm}$with $m q$ odd. By [31, proof of Proposition 4.2.16], we have $H_{\bar{I}}=$ $H_{\bar{\Omega}} \cong \mathrm{SO}_{m}(q) \times \mathrm{SO}_{m}(q)=\Omega_{m}(q) .2 \times \Omega_{m}(q) .2$, and $\operatorname{soc}\left(H_{\bar{I}}\right) \cong \Omega_{m}(q) \times \Omega_{m}(q)$. Furthermore, [31, Lemma 4.2.2(i)] implies that there exists an element $\sigma$ of $H_{\bar{\Delta}}$ such that $V_{1}^{\sigma}=V_{2}$. Hence, $\sigma$ interchanges the two copies of $\operatorname{SO}_{m}(q)$ in $H_{\bar{I}}$, from which it follows that $H_{\bar{\Delta}} / \operatorname{soc}\left(H_{\bar{I}}\right)$ is isomorphic to a subgroup of $D_{8}$. Since $\bar{H} / H_{\bar{\Delta}}$ is cyclic, we deduce that $\delta_{\bar{H}}(A)=1$ if $A$ is nonabelian; $\delta_{\bar{H}}(A) \leqslant 3$ if $A$ is central; and $\delta_{\bar{H}}(A) \leqslant 2$ if $A$ is abelian but noncentral.

It now follows that $\delta_{H}(A) \leqslant 1$ (respectively 2) if $A$ is nonabelian (respectively noncentral). If $A$ is central, then $\delta_{H}(A) \leqslant 4$, with equality if and only if $|A|=2$,

$(\star)$ holds and either

(1) case $\mathbf{L}$ holds or

(2) case $\mathbf{O}^{ \pm}$holds and either $m>1$ or $m=1$ and $q \equiv \pm 1(\bmod 8)$.

This completes the proof.

Proposition 6.8. Suppose that $H$ lies in $\mathcal{C}_{3}$. Then Theorem 2.7 holds.

Proof. This case is easier to handle than the previous ones. Suppose first that $H_{\bar{\Omega}}$ has a characteristic quasisimple subgroup $L$ with $C_{\bar{H}}(L)=Z(L)$. Then since it follows that $L \unlhd \bar{H}$, we have, by Lemma 3.5, that $Z(L) \leqslant \operatorname{Frat}(L) \leqslant \operatorname{Frat}(\bar{H})$. 
Thus, $\bar{H} / Z(L)$ is isomorphic to a subgroup of $\operatorname{Aut}(L / Z(L))$ containing $L / Z(L)$. In particular, $\delta_{\bar{H}}(A) \leqslant 1$ (respectively 2,3 ) if $A$ is nonabelian (respectively noncentral, central) for any non-Frattini chief factor of $\bar{H}$, by Proposition 3.12.

So we may assume that $H_{\bar{\Omega}}$ does not have a characteristic quasisimple subgroup $L$ with $C_{\bar{H}}(L)=Z(L)$. Then, by [31, Section 4.3], we must be in case $\mathbf{L}$ or $\mathbf{U}$, with $H$ of type $\operatorname{GL}_{1}^{\epsilon}\left(q^{n}\right)$. Then $H_{\Gamma} \leqslant \Gamma L_{1}\left(q^{u n}\right)$, which is metacyclic. Since $\left|H / H_{\Gamma}\right| \leqslant 2$, the result now follows immediately from Lemma 3.4.

Proposition 6.9. Suppose that $H$ lies in class $\mathcal{C}_{4}$. Then Theorem 2.7 holds.

Proof. Here, $H$ stabilizes a tensor product decomposition $V=V_{1} \otimes V_{2}$, and $\kappa=\kappa_{1} \otimes \kappa_{2}$, where $\kappa_{i}$ is either the zero form, a unitary form, a symplectic form or a symmetric form on $V_{i}$ (see [31, Table 4.4.A]). Also, $\left(V_{1}, \kappa_{1}\right)$ is not similar to $\left(V_{2}, \kappa_{2}\right)$. Let $I_{i}$ denote the group of isometries of $\left(V_{i}, \kappa_{i}\right)$, and, similarly, define $\Omega_{i}, S_{i}$ and $\Delta_{i}$ and the projective equivalents $\mathrm{PI}_{i}, \mathrm{P} \Omega_{i}, \mathrm{PS}_{i}$ and $\mathrm{P} \Delta_{i}$. Also, write $n_{i}:=\operatorname{dim}_{\mathbb{F}} V_{i}$ so that $n=n_{1} n_{2}$. We have $N_{\bar{I}}\left(V_{1} \otimes V_{2}\right) \cong\left(\mathrm{PI}_{1} \times \mathrm{PI}_{2}\right)\langle z\rangle$, where $z \in N_{\bar{S}}\left(V_{1} \otimes V_{2}\right)$ and $z^{2} \in \mathrm{PI}_{1} \times \mathrm{PI}_{2}$ (see [31, Lemma 4.4.5]).

Suppose first that we are not in case $\mathbf{O}$, and let $L:=\mathrm{P} \Omega_{1} \times \mathrm{P} \Omega_{2} \leqslant H_{\bar{\Omega}}$. Since the spaces $\left(V_{1}, \kappa_{1}\right)$ and $\left(V_{2}, \kappa_{2}\right)$ are nonisometric, the groups $\mathrm{P} \Omega_{i}$ are normal in $\bar{H}$. Hence, a chief factor $A$ of $\bar{H}$ contained in $L$ must be contained in one of the $\mathrm{P} \Omega_{i}$. Also, the only nonsimple groups $\mathrm{P} \Omega_{i}$ which can occur are $\mathrm{Sp}_{4}(2), \mathrm{L}_{2}(2)$, $\mathrm{L}_{2}(3), \mathrm{U}_{2}(2), \mathrm{U}_{2}(3), \mathrm{U}_{3}(2), \mathrm{PSp}_{2}(3), \Omega_{3}(3), \mathrm{P} \Omega_{4}^{+}$(3), by [31, Proposition 4.4.9]. Arguing as in the proof of Proposition 6.7, we can use the tables in $[9, \mathrm{Ch} .8]$ to show that at most one of these groups can occur.

Next, we consider the quotient $\bar{H} / L$. By [31, Lemma 4.4.3], $H_{\bar{\Delta}}$ induces diagonal automorphisms on $\mathrm{P} \Omega_{i}$, for each $i$. It is also clear that any field automorphism of $G_{0}$ induces a field automorphism on each P $\Omega_{i}$. Hence, $\bar{H} / L \leqslant$ $\left(\Gamma_{1} / \mathrm{P} \Omega_{1} \times \Gamma_{2} / \mathrm{P} \Omega_{2}\right) . R$, where $|R| \leqslant 2$ with equality if and only if we are in case $\mathbf{L}$ and $G$ contains a graph automorphism of $G_{0}$. Now, let us consider the intersection of $\bar{H} / L$ with the group $\Gamma_{1} / \mathrm{P} \Omega_{1} \times \Gamma_{2} / \mathrm{P} \Omega_{2}$. Call this intersection $Y$, and suppose first that we are not in case $\mathbf{O}$. Then the group $\Gamma_{i} / \mathrm{P} \Omega_{i}$ has shape $D_{i} . F_{i}$, where $D_{i}$ is cyclic of order dividing $\left(n_{i}, q^{u}-1\right)$ (we remind the reader that the integer $u$ is defined immediately after (6.2) on Page 26) and $F_{i}$ is cyclic of order dividing $u f$. Furthermore, by the arguments above, $Y / Y \cap\left(D_{1} \times D_{2}\right)$ is cyclic of order $e$, where $e$ is the order of the intersection of $G / G_{0}$ with the group of field automorphisms of $G_{0}$.

Suppose that we are in case $\mathbf{U}$ or $\mathbf{S}$. Then from the arguments in the above paragraph, we immediately get $\delta_{\bar{H}, \bar{H} / L}(A) \leqslant 3$, with equality possible only if $e>1$ and $A$ is central. Since $L$ is a direct product $L=\mathrm{P} \Omega_{1} \times \mathrm{P} \Omega_{2}$, where at least one of the groups is a nonabelian simple group, the result follows immediately 
unless one of the $\mathrm{P} \Omega_{i}$, say $\mathrm{P} \Omega_{1}$, is nonsimple. So assume that $\mathrm{P} \Omega_{1}$ is nonsimple. The possibilities for $\mathrm{P} \Omega_{1}$ are listed above, and we have $\delta_{\mathrm{P} \Omega_{1}}(B) \leqslant 2$ for all chief factors $B$ of $\mathrm{P} \Omega_{1}$, with equality if and only if $|B|=q, B$ is central and $\mathrm{P} \Omega_{1}=$ $\mathrm{U}_{3}(2)$ in case $\mathbf{U}$, or $\mathrm{P} \Omega_{1}=\mathrm{P} \Omega_{4}^{+}(3)$ in case $\mathbf{O}$. However, in these cases, the two central chief factors in question are fused by any noninner automorphism of $\mathrm{P} \Omega_{1}$. Thus, either $\delta_{\mathrm{P} \Omega_{1}}(B) \leqslant 1$ or $\delta_{\mathrm{P} \Omega_{1}}(B)=2$ and $e=1$. Thus, either way, we have $\delta_{\bar{H}}(A) \leqslant 3$. This gives us what we need unless $A$ is noncentral, so assume that this is the case. If $A$ is nonabelian, then we clearly have $\delta_{\bar{H}}(A)=1$, so assume also that $A$ is abelian. However, a quick computation with the possibilities for $I_{1}$ listed above shows that $\delta_{\mathrm{PI}_{1}}(B)=1$ for all noncentral chief factors $B$ of $\mathrm{PI}_{1}$. Since $H_{\bar{I}}$ is a subdirect product in $\overline{I_{1}} \cdot a \times \overline{I_{2}} \cdot a$, where $a \leqslant 2$, and $\bar{H} / H_{\bar{I}}$ is abelian, the bound $\delta_{\bar{H}}(A) \leqslant 2$ follows.

Thus, we may assume that we are in case $\mathbf{L}$ or case $\mathbf{O}$. Suppose first that we are in case $\mathbf{L}$. Then by arguing as in the above paragraph, we get that if $\mathrm{P} \Omega_{1}$ is nonsimple, then $\delta_{\bar{H}}(A) \leqslant 3$ (respectively 2,1 ) if $A$ is central (respectively noncentral, nonabelian). So we may assume that both $\mathrm{P} \Omega_{1}$ and $\mathrm{P} \Omega_{2}$ are nonabelian simple groups. The bound $\delta_{\bar{H}}(A) \leqslant 1$ if $A$ is nonabelian then follows immediately since $\mathrm{P} \Omega_{1}$ and $\mathrm{P} \Omega_{2}$ have distinct centralizers. So we may assume that $A$ is abelian. Then $\delta_{\bar{H}}(A)=\delta_{\bar{H} / L}(A)$, by Lemma 3.4. We now consider the structure of the group $\bar{H} / L$. First, $\bar{H} / H_{\bar{I}}$ is abelian, and as shown in the paragraph above, $H_{\bar{I}}$ is a subgroup of a group of shape $D_{1} \times D_{2}$, where $D_{i}$ is cyclic of order $\left(n_{i}, q-1\right)$. By Lemma 3.4, we then have $\delta_{\bar{H} / L}(A)=\delta_{\bar{H}, H_{\bar{T}} / L}(A)+$ $\delta_{\bar{H} / H_{\bar{I}}}(A)$. Since $\bar{H} / H_{\bar{I}}$ is abelian, the bound $\delta_{\bar{H}}(A) \leqslant 2$ (respectively 3 ) now follows immediately if $A$ is noncentral (respectively $A$ central with $|A| \neq 2$ ).

So we may assume that $|A|=2$. As shown in the fourth paragraph above, $Y:=(\bar{H} / L) \cap \bar{\Gamma}$ is a subgroup of a group of shape $D_{1} \cdot F_{1} \times D_{2} \cdot F_{2}$, where $D_{i}$ and $F_{i}$ are cyclic of order dividing $\left(n_{i}, q-1\right)$ and $f$, respectively. Also, setting $Y_{D}:=Y \cap\left(D_{1} \times D_{2}\right)$, we have that $Y / Y_{D}$ is cyclic of order $e$, isomorphic to the group of field automorphisms in $G$. Next, [31, Proposition 4.4.10] implies that the subgroup $C:=H_{\bar{\Omega}} / L$ of $Y \cap\left(D_{1} \times D_{2}\right)$ has order

$$
d:=\frac{\left(q-1, n_{1}\right)\left(q-1, n_{2}\right) \operatorname{hcf}\left(q-1, n_{1}, n_{2}\right)}{(q-1, n)} .
$$

Furthermore, $C$ contains $C_{1} \times C_{2}$, where $C_{i}$ is the unique subgroup of $D_{i}$ of order $\left(q-1, n_{1}, n_{2}\right)$. This is because if $\left(c_{1}, c_{2}\right) \in C \leqslant D_{1} \times D_{2}$, with $c_{i} \in D_{i}$, then $c_{1}^{n_{2}} c_{2}^{n_{1}}=1$, by [31, (4.4.9)]. Now, if either $n_{1}$ or $n_{2}$ is odd, or if $q$ is even, then $d$ is odd and we get $\delta_{\bar{H}}(A)=\delta_{\bar{H} / H_{\bar{\Omega}}}(A) \leqslant 3$, using Lemma 6.4. So we may assume that $n_{1}$ and $n_{2}$ are even, and that $q$ is odd. Then $Y_{D}=H_{\bar{I}} / L$ is a subgroup of $D_{1} \times D_{2}$ containing a subgroup $C_{1} \times C_{2}$ with $\left|C_{i}\right|$ even. Hence, $Y_{D} /\left(\operatorname{Frat}\left(Y_{D}\right) Y_{D}^{2}\right)$ is an 
$\bar{H}$-module of order $2^{2}$. In fact, using the relations on $\operatorname{Out}\left(G_{0}\right)$ from [31, Section 2], we see that this is a trivial $\left(\bar{H} / H_{\bar{\Omega}}\right)$-module. Since $\delta_{\bar{H} / H_{\bar{I}}}(A)=\delta_{2,(e, 2)}+i$, where $i:=1$ if $G$ contains a graph automorphism, and $i:=0$ otherwise, we deduce from Lemma 3.4 that $\delta_{\bar{H}}(A) \leqslant 4$, with equality if and only if $n_{1}$ and $n_{2}$ are even, $q$ is odd and $G$ contains both a field automorphism of even order and a graph automorphism.

Finally, assume that we are in case $\mathbf{O}$. Suppose first that $H$ has type $\mathrm{O}_{n_{1}}^{\epsilon_{1}}(q) \otimes$ $\mathrm{O}_{n_{2}}^{\epsilon_{2}}(q)$ with $q$ odd, $n_{i} \geqslant 3$ and $\left(n_{1}, \epsilon_{1}\right) \neq\left(n_{2}, \epsilon_{2}\right)$. Write $Q_{i}$ for the quadratic form associated with $\kappa_{i}$.

Now, similar to case $\mathbf{L}$, we have that $H_{\bar{\Delta}} / L$ is a subgroup of $\left(\mathrm{P} \Delta_{1} / \mathrm{P} \Omega_{1}\right) \times$ ( $\mathrm{P} \Delta_{2} / \mathrm{P} \Omega_{2}$ ), while $\bar{H} / H_{\bar{\Delta}}$ is cyclic of order $e \mid f$. Thus, all non-Frattini chief factors of $\bar{H} / L$ are central. Since we also have $\delta_{\mathrm{P} \Omega_{i}}(B) \leqslant 2$ for all non-Frattini chief factors $B$ of $\mathrm{P} \Omega_{i}$, we have that $\delta_{\bar{H}}(A)=\delta_{\bar{H}, L}(A) \leqslant 2$ if $A$ is noncentral. It is also clear that $\delta_{\bar{H}}(A)=1$ if $A$ is nonabelian. So we may assume that $A$ is central. Since all central chief factors in the possible nonsimple groups $\mathrm{P} \Omega_{i}$ are acted upon nontrivially by noninner automorphisms, we quickly reduce to the case where $\delta_{\bar{H}}(A)=\delta_{\bar{H} / L}(A)$.

We now consider the structure of $\bar{H} / L$. If $n$ is odd, then $\bar{H} / L$ has shape $R_{1} . R_{2}$.e, where $\left|R_{i}\right| \leqslant 2$ and $e \mid f$, by [31, Proposition 4.4.18]. The result then follows immediately from Lemma 3.4. So we may assume that $n$ is even. Clearly, we may also assume that $|A|=2$. Now, as above, we have that $H_{\bar{\Delta}} / L$ is a subgroup of $\left(\mathrm{P} \Delta_{1} / \mathrm{P} \Omega_{1}\right) \times\left(\mathrm{P} \Delta_{2} / \mathrm{P} \Omega_{2}\right)$, while $\bar{H} / H_{\bar{\Delta}}$ is cyclic of order $e \mid f$.

We now determine the shape of the group $H_{\bar{\Omega}} / L \leqslant\left(\mathrm{P} \Delta_{1} / \mathrm{P} \Omega_{1}\right) \times\left(\mathrm{P} \Delta_{2} / \mathrm{P} \Omega_{2}\right)$. We define $s_{\epsilon_{i}}:=1$ if $\epsilon_{i}=+$ and $s_{\epsilon_{i}}:=0$ otherwise. Then by [31, Lemma 4.4.13], $H_{\bar{\Omega}} / L \leqslant\left(\mathrm{P} \Delta_{1} / \mathrm{P} \Omega_{1}\right) \times\left(\mathrm{P} \Delta_{2} / \mathrm{P} \Omega_{2}\right)$ has shape:

(1) $1 \times Z_{2}$ if $n_{2}$ is odd;

(2) $2^{2} \times 2^{2}$ if $\left(n_{1}, n_{2}\right)$ is even and both $Q_{i}$ have discriminants that are square;

(3) $2 \times 2^{(2, i+2)}$ if $\left(n_{1}, n_{2}\right)$ is even, $Q_{1}$ has square discriminant and $Q_{2}$ has nonsquare discriminant with Witt index $i \in\{0,1\}$;

(4) $\left(2^{s_{\epsilon_{1}}} \times 2^{s_{\epsilon_{2}}}\right) \cdot 2$ (a subdirect product of $\left.2^{1+s_{\epsilon_{1}}} \times 2^{1+s_{\epsilon_{2}}}\right)$ if $\left(n_{1}, n_{2}\right)$ is even and both discriminants are nonsquare.

Since $\mathrm{P} \Delta_{2} / \mathrm{P} \Omega_{2}=Z_{2}$ in case $(1)$, we have that $\delta_{\bar{H}}(A)=1+\delta_{\bar{H} / H_{\bar{\Omega}}}(A) \leqslant 4$, with equality if and only if $G / G_{0}$ intersects $\bar{\Delta} / \bar{\Omega}$ in a noncyclic group and $e$ is even. Note, in particular, that these conditions can only hold if $(\star)$ holds (see Page 3 for the definition of $(\star))$ and either $\bar{H}$ is of type $\mathrm{O}_{n_{1}}^{+}(q) \otimes \mathrm{O}_{n_{2}}(q)$ or of type $\mathrm{O}_{n_{1}}^{-}(q) \otimes \mathrm{O}_{n_{2}}(q)$ with $Q_{1}$ having square discriminant. 
This leaves us to deal with cases (2)-(4). If we are in case (2), then $H_{\bar{I}}=H_{\bar{\Omega}}$ by [31, Lemma 4.4 .13 (ii)(b)]. Thus, if $H_{\bar{\Delta}}=H_{\bar{I}}$, then $\delta_{\bar{H}}(A)=4+\delta_{2,(2, e)}$. If $H_{\bar{\Delta}}>H_{\bar{I}}$, then $H_{\bar{\Delta}} / H_{\bar{\Omega}}$ is a proper subdirect product of $D_{8} \times D_{8}$ containing $2^{2} \times 2^{2}$. It follows easily that the intersection of each factor with the centre of the relevant copy of $D_{8}$ is Frattini. Hence, we get $\delta_{\bar{H}}(A)=3+\delta_{2,(2, e)}$. If we are in case (3), then $H_{\bar{S}}=H_{\bar{I}}$, again by [31, Lemma 4.4.13 (ii)(b)]. We then get that $\delta_{\bar{H}}(A)=2+\delta_{2,(2, i+2)}+\delta_{2,(2, e)}\left(\right.$ respectively $\left.\delta_{\bar{H}}(A)=1+\delta_{2,(2, i+2)}+\delta_{2,(2, e)}\right)$ if $G / G_{0}$ intersects $\bar{\Delta} / \bar{\Omega}$ nontrivially (respectively trivially). Finally, if case (4) holds, then arguing as above, we get $\delta_{\bar{H}}(A)=2+s_{\epsilon_{1}}+s_{\epsilon_{2}}+\delta_{2,(2, e)}$ (respectively $\left.\delta_{\bar{H}}(A)=1+s_{\epsilon_{1}}+s_{\epsilon_{2}}+\delta_{2,(2, e)}\right)$ if $G / G_{0}$ intersects $\bar{\Delta} / \bar{\Omega}$ nontrivially (respectively trivially).

The argument is entirely similar when we are in case $\mathbf{O}^{+}$with $H$ of type $\operatorname{Sp}_{n_{1}}(q) \otimes \operatorname{Sp}_{n_{2}}(q)$. In fact, we get $\delta_{\bar{H}}(A) \leqslant 2$ for all $A$ in this case. This completes the proof.

Proposition 6.10. Suppose that $H$ lies in class $\mathcal{C}_{5}$. Then Theorem 2.7 holds.

Proof. In this case, $H$ stabilizes a form $\kappa_{1}$ on a vector space $V_{1}$ of dimension $n$ over a subfield $\mathbb{F}_{1}$ of $\mathbb{F}$ of prime index $r$. Let $I_{1}$ be the group of isometries of $\left(V_{1}, \kappa_{1}\right)$. Also define $\Omega_{1}, \Delta_{1}, \mathrm{P} \Omega_{1}$ and $\mathrm{P} \Delta_{1}$ in the usual way. Then $H_{\bar{\Gamma}} \leqslant$ $N_{\bar{\Gamma}}\left(V_{1}\right) \cong \mathrm{P} \Delta_{1} \rtimes\left\langle\phi_{r}\right\rangle$, where $\phi_{r}$ is a generator for $\operatorname{Gal}\left(\mathbb{F}: \mathbb{F}_{1}\right)$. It follows that the centralizer in $\bar{H}$ of $\bar{\Omega}_{1}$ is contained in $\left\langle\phi_{r}\right\rangle$ and, hence, that $\bar{H} / H_{\bar{\Omega}}$ is isomorphic to a subgroup of $\operatorname{Out}\left(\mathrm{P} \Omega_{1}\right) . r$, where the extension is a cyclic extension of the associated group of field automorphisms of $\Omega_{1}$. Thus, $\bar{H} / H_{\bar{\Omega}}$ is an extension of at most three cyclic groups and has at most one noncentral chief factor. The bound $\delta_{\bar{H}}(A) \leqslant 1$ (respectively 2,3 ) now follows immediately if $\mathrm{P} \Omega_{1}^{\prime}$ is nonabelian simple and $A$ is nonabelian (respectively noncentral, central). Otherwise, the list of possibilities for $\mathrm{P} \Omega_{1}^{\prime}$ is in [31, Proposition 2.9.2], and the desired bound follows quickly by direct computation.

Proposition 6.11. Suppose that $H$ lies in class $\mathcal{C}_{6}$. Then Theorem 2.7 holds.

Proof. Here, $n=r^{m}$ for a prime $r$, and the group $H_{\bar{\Delta}}$ is a subgroup of $\overline{N_{\Delta}(R)}$ for an extraspecial $r$-group $R \leqslant \Gamma$ (see [31, Definition, Page 150]). By [31, (4.6.1) and Table 4.6.A], the group $\overline{N_{\Delta}(R)}$ has shape $\bar{R} . C$, where $\bar{R} \cong r^{2 m}$ and either $C \cong \mathrm{Sp}_{2 m}(r)$ or $r=2$ and $C \cong O_{2 m}^{ \pm}(2)$. Also, $C$ acts naturally on $\bar{R}$. Furthermore, $\bar{R}$ is contained in $H_{\bar{\Omega}}$, and $H_{\bar{\Omega}} / \bar{R}$ acts irreducibly on $\bar{R}$. Since $H_{\bar{\Omega}}$ is normal in $\bar{R}$.C, it follows that $H_{\bar{\Delta}}$ has shape $\bar{R}$. $C_{1}$, where $C_{1}$ is a subgroup of $C$ containing a (nontrivial) normal irreducible subgroup of $C$.

Suppose first that $n \geqslant 4$. Then $C / Z(C)$ is almost simple, and $C^{\prime}$ is quasisimple. Furthermore, $C / C^{\prime}$ is cyclic. It follows that $\delta_{\bar{H}, H_{\bar{\Delta}}}(A) \leqslant 1$ (recall that the centre 
of a quasisimple group is Frattini; see Lemma 3.5). Since $\bar{H} / H_{\bar{\Delta}}$ is abelian of rank at most 2 , it follows that $\delta_{\bar{H}}(A) \leqslant 1$ (respectively 2,3 ) if $A$ is nonabelian (respectively noncentral, central) for any non-Frattini chief factor of $\bar{H}$, which gives us what we need.

Next, assume that $n=3$ so that $(r, m)=(3,1)$. Then $H_{\bar{\Delta}}$ has shape $3^{2} . C_{1}$, where $C_{1} \in\left\{Q_{8}, \mathrm{Sp}_{2}(3)\right\}$ (see [31, Proof of Proposition 4.6.4]). It follows that $\delta_{\bar{H}, H_{\bar{\Delta}}}(A) \leqslant 1$, and the required bounds follow as in the previous paragraph. The case $n=2$ is similar.

Proposition 6.12. Suppose that $H$ lies in class $\mathcal{C}_{7}$. Then Theorem 2.7 holds.

Proof. Here, $H_{\Gamma}$ is a subgroup of the stabilizer in $\Gamma$ of a tensor decomposition

$$
V=V_{1} \otimes V_{2} \otimes \cdots \otimes V_{t}
$$

where $\kappa=\kappa_{1} \otimes \cdots \otimes \kappa_{t}$ for appropriate forms $\kappa_{i}$ on $V_{i}$ (see [31, Table 4.7.A]). Furthermore, the spaces $\left(V_{i}, \kappa_{i}\right)$ are isometric, so they all have a common dimension $m$. In particular, $n=m^{t}$. Write $I_{i}$ for the group of isometries of $\left(V_{i}, \kappa_{i}\right)$. Similarly, define $\Omega_{i}$ and $\Delta_{i}$ and the projective equivalents $\mathrm{P} \Omega_{i}, \mathrm{P} \Delta_{i}$ and $\mathrm{PI}_{i}$.

Let $\mathcal{D}=\left\{V_{1}, \ldots, V_{t}\right\}$, and write $\Gamma_{\mathcal{D}}$ for the full (setwise) stabilizer in $\Gamma$ of $\mathcal{D}$. Also, for any subgroup $K$ of $\Gamma_{\mathcal{D}}$, write $K_{(\mathcal{D})}$ for the kernel of the action of $K$ on $\mathcal{D}$. Also, write $K^{\mathcal{D}}=K / K_{(\mathcal{D})} \leqslant \operatorname{Sym}_{t}$ for the induced action of $K$ on $\mathcal{D}$. We then have a normal series

$$
1 \leqslant H_{\overline{\Delta(\mathcal{D})}} \leqslant H_{\bar{\Delta}} \leqslant \bar{H} .
$$

Thus, we have

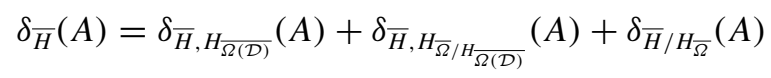

using Lemma 3.4. Now, since $G$ contains $\Omega, H_{\Delta}$ contains the full setwise stabilizer $\Omega_{\mathcal{D}}$ of $\mathcal{D}$ in $\Omega$. By [31, (4.7.1) and (4.7.2)], we have

$$
\overline{\Delta_{\mathcal{D}}}=\overline{\Delta_{(\mathcal{D})}} J \cong \mathrm{P} \Delta_{1} 2 \operatorname{Sym}_{t}
$$

(with the product action), where $\overline{\Delta_{(\mathcal{D})}} \cong\left(\mathrm{P} \Delta_{1}\right)^{t}$ and $J \cong \mathrm{Sym}_{t}$. Furthermore, by [31, (4.7.8)], the intersection $J_{\bar{\Omega}}$ is either $\mathrm{Alt}_{t}$ or $\mathrm{Sym}_{t}$.

Now, the group $H_{\overline{\Omega_{(\mathcal{D})}}}$ is contained in $\mathrm{P} \Delta_{1}^{t}$ and contains the subgroup $\mathrm{P} \Omega_{1}^{t}$. Also, $N_{\bar{H}}\left(\mathrm{P} \Delta_{1}\right)=\operatorname{Stab}_{\bar{H}}\left(V_{1}\right)$.

Assume first that $\bar{H}^{\mathcal{D}}$ is transitive. Then $\bar{H}=N_{\bar{H}}\left(\mathrm{P} \Delta_{1}\right) H_{\bar{\Delta}}$. The other hypotheses required in Lemma 3.9 follow from Lemma 3.10 in this case.

Thus, we may apply Lemma 3.9 to find the chief factors of $\bar{H}$ contained in $H_{\overline{\Omega_{(\mathcal{D})}}}$. In fact, the argument here is exactly the same as in the proof of 
Proposition 6.7: we have $\delta_{\bar{H}, H_{\bar{\Omega}_{(\mathcal{D})}}}(A) \leqslant 1$, with equality possible only if either $A$ is noncentral or $|A|=2$ and $\mathrm{P} \Omega_{1}=\mathrm{Sp}_{4}(2)$.

Thus, we have determined the quantity $\delta_{\bar{H}, H_{\bar{\Omega}_{(\mathcal{D})}}}(A)$. We now determine the other quantities in the bound (6.16). As shown above, $\bar{H} / H_{\overline{\Omega^{\mathcal{D}}}} \cong J_{\bar{\Omega}} \cdot\left(\bar{H} / H_{\bar{\Omega}}\right)$, and $J_{\bar{\Omega}}=$ Sym $_{t}$ unless one of the following holds (see [31, Section 4.7]):

(1) case $\mathbf{L}, t=2, m \equiv 2(\bmod 4)$ and $q \equiv-1(\bmod 4)$;

(2) case $\mathbf{U}, t=2, m \equiv 2(\bmod 4)$ and $q \equiv 1(\bmod 4)$;

(3) case $\mathbf{O}^{+}, t=2, m \equiv 2(\bmod 4)$; or

(4) case $\mathbf{O}^{+}, H$ of type $\mathrm{O}_{m}^{\epsilon}(q) 2 \operatorname{Sym}_{t}, \epsilon \in\{ \pm\}, t=3, m \equiv 2(\bmod 4)$ and $q \equiv 3 \epsilon(\bmod 4)$.

Now, arguing as in the proof of Proposition 6.7, we have that the $Z_{2}$ chief factor of $\bar{H} / H_{\overline{\Omega^{\mathcal{D}}}}$ is non-Frattini (and central). Thus, we conclude that $\delta_{\bar{H}, H_{\overline{\Omega^{\mathcal{D}}}}}(A) \leqslant 1$, with equality if and only if

(a) $|A|=2$ (so that $A$ is central) and we are not in cases (1)-(4) above; or

(b) $A \cong \mathrm{Alt}_{t}$ and $t \neq 2,4$;

(c) $t=4$ and $|A| \in\{3,4\}$.

Finally, by applying Lemma 6.4 , we deduce that $\delta_{\bar{H}, \bar{H} / H_{\bar{\Omega}}}(A) \leqslant 3$, with equality if and only if $|A|=2$ and $(\star)$ holds (see Page 3 for the definition of $(\star)$ ).

Assume next that $\bar{H}^{\mathcal{D}}$ is intransitive. Then $t=2$ and $\bar{H}^{\mathcal{D}}=1$. Since $\bar{\Delta}$ induces Sym $_{2}$ on $\mathcal{D}$, we must have that $H_{\bar{\Delta}} \leqslant H_{\overline{\Delta(\mathcal{D})}}$. Arguing in a similar way to the two previous paragraphs, it then follows that $\bar{H}$ has shape $\left(\mathrm{P} \Omega_{1} \times \mathrm{P} \Omega_{2}\right) . C . R$, where $C$ is either cyclic (if we are not in case $\mathbf{O}$ ) or isomorphic to a subgroup of $D_{8}$. The group $R$ is cyclic in case $\mathbf{O}$, and a direct product of (possibly trivial) cyclic groups otherwise. The bound $\delta_{\bar{H}}(A) \leqslant 3$, with equality possible only if $|A|=2$, now follows as above. Furthermore, $\delta_{H}(A) \leqslant 1$ (respectively 2 ) if $A$ is nonabelian (respectively noncentral).

It now follows that $\delta_{H}(A) \leqslant 1$ (respectively 2) if $A$ is nonabelian (respectively noncentral). If $A$ is central, then $\delta_{H}(A) \leqslant 4$, with equality if and only if $|A|=2$, $(\star)$ holds and either

(i) case $\mathbf{L}$ holds and we are not in case 1 above or

(ii) case $\mathbf{O}^{ \pm}$holds and we are not in cases (3)-(4) above.

This completes the proof. 
Proposition 6.13. Suppose that $H$ lies in class $\mathcal{C}_{8}$. Then Theorem 2.7 holds.

Proof. The argument here is almost identical to the argument used to prove Proposition 6.10: we repeat the details here for the reader's benefit. The group $H_{\Gamma}$ here is a classical group itself of dimension $n$ over $\mathbb{F}$ : we write $\kappa_{1}$ for the associated unitary, symplectic or quadratic form. See [31, Table 4.8.A] for the precise possibilities for $H_{\Gamma}$.

Let $I_{1}$ be the group of isometries of $\left(V, \kappa_{1}\right)$, and similarly define $\Omega_{1}$ and the projective groups $\mathrm{PI}_{1}$ and $\mathrm{P} \Omega_{1}$. The group $\mathrm{P} \Omega_{1}$ is normal in $\bar{H}$ by [31, Proof of Proposition 4.8.2]. Furthermore, $\Omega_{1}$ is absolutely irreducible by [31, Proposition 2.10.6]. Hence, $C_{H}\left(\Omega_{1}\right)=\mathbb{F}^{*}$. It follows that $C_{H_{\bar{I}}}\left(\mathrm{P} \Omega_{1}\right)$ is trivial and, hence, that $H_{\bar{I}} / \mathrm{P} \Omega_{1} \leqslant \operatorname{Out}\left(\mathrm{P} \Omega_{1}\right)$. If $\mathrm{P} \Omega_{1} \cong \mathrm{Sp}_{4}(2)$, then $\bar{H} \leqslant \operatorname{Aut}\left(\mathrm{Alt}_{6}\right)=\mathrm{Alt}_{6} \cdot 2^{2}$ and $\delta_{\bar{H}}(A) \leqslant 2$.

Now, assume that $\mathrm{P} \Omega_{1}$ is a nonabelian simple group. Then $H_{\bar{\Gamma}} / H_{\bar{I}}$ induces a field automorphism on $\mathrm{P} \Omega_{1}$, so $H_{\bar{\Gamma}}$ is almost simple, isomorphic to a subgroup of $\Gamma_{1}$. It follows that either $\bar{H}$ is almost simple or of the form $\bar{H}=K \times Z_{2}$, where $K \leqslant \Gamma_{1}$ is almost simple. The required bounds $\delta_{\bar{H}}(A) \leqslant 1$ (respectively 2,3 ) if $A$ is nonabelian (respectively noncentral, central) follow from Proposition 3.12.

Finally, if $\mathrm{P} \Omega_{1}$ is not a nonabelian simple group, then we can deduce the possibilities for $\bar{H}$ from [31, Proposition 2.9.2]. The required bounds follow easily by direct computation in each case.

\section{The exceptional and remaining classical cases}

In this final section, we deal with the cases where the socle $G_{0}$ of $G$ is either an exceptional simple group or in $\left\{\operatorname{Sp}_{4}\left(2^{f}\right), \mathrm{P} \Omega_{8}^{+}(q)\right\}$. Furthermore, in the cases when $G_{0} \in\left\{\operatorname{Sp}_{4}\left(2^{f}\right), \mathrm{P} \Omega_{8}^{+}(q)\right\}$, we may (and will) assume that $G \nless \bar{\Gamma}$, where $\bar{\Gamma}$ is as defined in Section 6 . We begin by fixing some notation. Let $\mathbf{G}$ be a simple algebraic group of adjoint type over an algebraically closed field $\mathbb{F}$ of characteristic $p>0$, and let $\sigma$ be a Steinberg endomorphism of G. Denote the (finite) fixed point subgroup of $\mathbf{G}$ under $\sigma$ by $\mathbf{G}_{\sigma}$. In this section, we will assume that $G_{0}:=\left(\mathbf{G}_{\sigma}\right)^{\prime}$ is either an exceptional finite simple group or isomorphic to $\operatorname{Sp}_{4}(q)$ or $\mathrm{P} \Omega_{8}^{+}(q)$. Let $\Phi$ be a root system for $\mathbf{G}$, and let $\Pi:=\left\{\alpha_{1}, \ldots\right.$, $\alpha_{r}$ \} be a set of fundamental roots, where $r$ denotes the Lie rank of $\mathbf{G}$. We label the fundamental roots, and the corresponding Dynkin diagram for $\mathbf{G}$, as in Bourbaki [8].

By [26, Theorem 2.2.3], we may assume that $\sigma$ has the form $\sigma=g_{\rho} \phi_{q}$, where $g_{\rho}$ is a (possibly trivial) graph automorphism of $\mathbf{G}$ induced by a bijection $\rho$ of $\Pi$ and $\phi_{q}$ is a Frobenius endomorphism of $\mathbf{G}$. In this case, we write $G_{0}={ }^{d} \mathbf{G}\left(q^{2 u+1}\right)$, where $d$ is the order of the automorphism $g_{\rho}, u$ is defined to be 1 if $\mathbf{G}=B_{2}, F_{4}$ 
or $G_{2}$ and $G_{0}$ is twisted (that is, $g_{\rho}$ is nontrivial) and $u:=0$ otherwise. We also write $\tau$ for the linear map induced on the Euclidean space $\mathbb{R} \Phi$ by the bijection $\rho$ above. Also, we fix a $\sigma$-invariant maximal torus $\bar{T} \cong \mathbb{F}^{r}$ in $\mathbf{G}$.

Throughout, we will write $H_{0}:=H \cap G_{0}$. Also, for a subgroup $X$ of $\mathbf{G}$, we will write $X_{\sigma}$ for the group $X \cap \mathbf{G}_{\sigma}$. Finally, we will write $I=\operatorname{Inndiag}\left(G_{0}\right)=\mathbf{G}_{\sigma}$ for the group of inner diagonal automorphisms of $G_{0}$.

We begin with the classical cases which were omitted in Section 6.

Proposition 7.1. Suppose that $G_{0} \in\left\{\operatorname{Sp}_{4}\left(2^{f}\right), \mathrm{P} \Omega_{8}^{+}(q)\right\}$ and that $G \nless \bar{\Gamma}$. Then Theorem 2.7 holds.

Proof. Let $g \in G$ be an exceptional automorphism of $G_{0}$, in the language of Remark 6.3. Then $g$ reduces to a graph automorphism $g_{\rho}$ modulo field and inner diagonal automorphisms. The automorphism $g_{\rho}$ has order $|\rho|=2$ if $G_{0}=\operatorname{Sp}_{4}\left(2^{f}\right)$ and order $|\rho|=3$ if $G=\mathrm{P} \Omega_{8}^{+}(q)$ (in the latter case, $g$ is said to be a triality automorphism). Adopting the notation above, we note first that by [26, Theorem 2.5.1], $g$ acts on the maximal torus $\bar{T} \cong\left(\mathbb{F}^{\times}\right)^{r}$ as an element of $\operatorname{Aut}\left(\mathbb{F}^{\times}\right)$\& $\operatorname{Sym}_{r}$, projecting on to a $|\rho|$-cycle in $\operatorname{Sym}_{r}$. Furthermore, $g_{\rho}$ acts on the root subgroups $U_{\alpha}(\alpha \in \Phi)$ in the obvious way, by sending $U_{\alpha}$ to $U_{\alpha^{\rho}}$.

Suppose first that $G_{0}=\operatorname{Sp}_{4}(q)$, where $q=2^{f}$. Here, $\left|g_{\rho}\right|=2$. The maximal subgroups of $G$ were determined by Aschbacher [1], and most are groups which have already been dealt with in Section 6. The ones we have not dealt with are those for which $H_{0}$ is not maximal in $G_{0}$. In these cases, $G$ contains an exceptional automorphism $g$ as in the first paragraph above. By [1, Theorem 14.2], the possibilities for $H_{0}$ are as follows:

(i) $H_{0}$ has shape $C \rtimes(q-1)^{2}$, where $C$ is a 2-group and $C / C^{\prime} \cong\left(\mathbb{F}_{q}\right)^{2}$;

(ii) $H_{0}$ has shape $\left(\Omega_{2}^{\epsilon}(q)^{2} .2\right) .2$; or

(iii) $H_{0}$ has shape $\Omega_{2}^{-}\left(q^{2}\right)^{2} \cdot 2$.

In the first case, $H_{0} / C^{\prime} \cong\left(\mathbb{F}_{q} \rtimes \mathbb{F}_{q}^{\times}\right)^{2}$ is a direct product of affine groups, and $g$ switches the two factors (see the first paragraph above). It follows that $C / C^{\prime}$ is irreducible as an $\mathbb{F}_{2}[H]$-module. Hence, $C / C^{\prime}$ is the unique non-Frattini chief factor of $\bar{H}$ contained in $C$. Furthermore, the non-Frattini chief factors of $H$ contained in $H_{0} / C$ can be described as follows: let $l$ be a prime divisor of $q-1$ (in particular, $l$ is odd). If $g^{2}$ acts irreducibly on the natural $\left(Z_{l}\right)^{2}$ factor of $\left(\mathbb{F}_{q}^{\times}\right)^{2}$, then $\left(Z_{l}\right)^{2}$ is the unique non-Frattini $l$-chief factor of $H$ contained in the section $\left(\mathbb{F}_{q}^{\times}\right)^{2}$. If $g^{2}$ does not act irreducibly, then the $\left(Z_{l}\right)^{2}$ comprises two non-Frattini chief factors of $H$ : one is trivial of order $l$, while the other is the deleted subgroup 
of $\left(Z_{l}\right)^{2}$ (nontrivial). Thus, since $H / H_{0}$ is cyclic, we deduce that $\delta_{H}(A) \leqslant 2$ in all cases.

In case (ii), the 2 on top of $H_{0}$ acts by switching the two factors of $\Omega_{2}^{\epsilon}(q)^{2}$. Arguing in a similar way to the previous paragraph, we see that the non-Frattini chief factors of $\bar{H}$ contained in $\Omega_{2}^{\epsilon}(q)^{2}$ have orders 2 , and either $l$ or $l^{2}$, for each prime $l$ dividing $q-\epsilon$. Since $H / H_{0}$ is cyclic, we deduce that $\delta_{H}(A) \leqslant 2$.

In case (iii), we have $H_{0} \cong D_{2\left(q^{2}+1\right)} .2$, and the non-Frattini chief factors of $H$ contained in $H_{0}$ have orders 2 (with multiplicity 2 ) and $l$, for each prime $l$ dividing $q^{2}+1$. Hence, $\delta_{H}(A) \leqslant 3$, with equality possible only if $|A|=2$.

Finally, assume that $G_{0}=\mathrm{P} \Omega_{8}^{+}(q)$. The maximal subgroups of $G$ were determined by Kleidman [30], and as above, the only ones we have not dealt with are those for which $H_{0}$ is not maximal in $G_{0}$. Using [30, Table III], the possible groups are as follows:

(iv) $H_{0}$ has shape $C \rtimes L$, where $C$ is a $p$-group, $C / C^{\prime} \cong\left(\mathbb{F}_{q}\right)^{6}$ and $L$ is a subdirect product of $\mathrm{GL}_{2}(q) \times(q-1) \times(q-1)$;

(v) $H_{0}$ has shape $\left(1 /(2, q-1) \mathrm{GL}_{3}^{\epsilon}(q) \times(q-\epsilon) /(2, q-1)\right) .2^{2}$, where $1 /(2, q-1) \mathrm{GL}_{3}^{\epsilon}(q)$ is the unique subgroup of $\mathrm{GL}_{3}^{\epsilon}(q)$ of index $(2, q-1)$ containing $\mathrm{SL}_{3}^{\epsilon}(q)$;

(vi) $H_{0} \cong G_{2}(q)$;

(vii) $H_{0}$ has shape $\left(D_{2\left(q^{2}+1\right) /(2, q-1)} \times D_{2\left(q^{2}+1\right) /(2, q-1)}\right) \cdot 2^{2}$; or

(viii) $q$ is odd and $H_{0}$ has shape $\left(2^{6} \cdot 2^{3}\right) \cdot \mathrm{L}_{3}(2)$.

Suppose first that we are in case (iv), and write $\Pi=\left\{\alpha_{1}, \alpha_{2}, \alpha_{3}, \alpha_{4}\right\}$ so that $\Pi$ is a set of fundamental roots for $\mathbf{G}$ as outlined in the notation introduced preceding the proposition. Using [26, Sections 1.13 and 1.14], we can see that $C$ is generated by the root subgroups $U_{\alpha}$, where $\alpha=\sum_{i=1}^{4} c_{i} \alpha_{i}$ with $c_{1}+c_{3}+c_{4}=1$. In turn, it is easy to see that

$$
C=C^{\prime}\left\langle U_{\alpha_{1}}, U_{\alpha_{1}+\alpha_{2}}\right\rangle+C^{\prime}\left\langle U_{\alpha_{3}}, U_{\alpha_{3}+\alpha_{2}}\right\rangle+C^{\prime}\left\langle U_{\alpha_{4}}, U_{\alpha_{4}+\alpha_{2}}\right\rangle .
$$

The triality automorphism then acts as a 3-cycle on the set $\left\{U_{\alpha_{1}}, U_{\alpha_{3}}, U_{\alpha_{4}}\right\}$ (see the first paragraph of the proof above). Since $\mathrm{GL}_{2}(q)$ acts naturally on the two-dimensional subspace $C^{\prime}\left\langle U_{\alpha_{1}}, U_{\alpha_{1}+\alpha_{2}}\right\rangle / C^{\prime}$ (see [30, Section 4.1.4]), we deduce that $H$ acts irreducibly on $C / C^{\prime}$. We also have that $L$ has structure $\mathrm{SL}_{2}(q) .\left(C_{1} \times C_{2} \times C_{3}\right)$, where the $C_{i}$ are cyclic groups of order dividing $q-1$, permuted transitively by the triality automorphism in $H$. Suppose $q>3$. Then it follows as in the $\operatorname{Sp}_{4}(q)$ case above that the non-Frattini chief factors of $H$ contained in $H_{0}$ form a subset of $\left\{q^{6}, \mathrm{~L}_{2}(q), l, l^{2}, 3: l \mid q-1, l\right.$ prime, $\left.l \neq 3\right\}$. 
Furthermore, $\delta_{H, H_{0}}(A)=1$ in each case. Since $H / H_{0} \leqslant \operatorname{Sym}_{4} \times Z_{f}$, we have $\delta_{H, H / H_{0}}(A) \leqslant 2$, with equality possible only if $A$ is central. The required bounds now follow immediately from Lemma 3.4. If $q \leqslant 3$, then the result can be quickly checked using Magma.

Suppose next that case (v) holds. If $q=2$, then the result is easily checked, so assume that $q>2$. Then using [26, Section 1.13], we see that $H_{0} / H_{0}^{\prime}$ is conjugate to a subquotient of the torus $\bar{T}$ in $\mathbf{G}$. Since $\bar{T}$ itself is acted upon via permutation of coordinates by a triality automorphism (see the first paragraph of the proof), we can use similar arguments to the $\operatorname{Sp}_{4}(q)$ case above to determine the nonFrattini chief factors of $H$ contained in $H_{0}$. We have that $\delta_{H, H_{0}}(A)=1$ for any non-Frattini chief factor of $H$ contained in $H_{0}$. Since $H / H_{0} \leqslant \operatorname{Sym}_{4} \times Z_{f}$, the result now follows as in the previous paragraph.

Next, suppose that case (vi) holds. That is, $H_{0} \cong G_{2}(q)$. Then $H_{0}^{\prime}$ is simple of index 1 or 2 in $H_{0}$. If the index is 1 , then $H / H_{0}^{\prime} \leqslant \operatorname{Aut}(G) \leqslant Z_{f} \times \operatorname{Sym}_{4}$ and the required bounds follow. If the index is 2 then $q=2$ and $H / H_{0}^{\prime} \leqslant 2 . \mathrm{Sym}_{4}$, and the required bounds again follow.

The argument in case (vii) is similar to case (v). Let $N:=D_{2\left(q^{2}+1\right) /(2, q-1)} \times$ $D_{2\left(q^{2}+1\right) /(2, q-1)}$. Then we have that $N=C^{2} \cdot 2^{2}$ with $C$ cyclic, and the section $C^{2}$ is conjugate to a section of the torus $\bar{T}$. The $2^{2}$ on top of $N$ also satisfies this property if $p \neq 2$. Thus, arguing as in the previous cases, we have $\delta_{H, N}(A) \leqslant 2$, with equality possible only if $p=2$ and $|A|=2$. By using [30, Proposition 3.3.1 and its proof] again, we also have that if $P$ is a Sylow 2-subgroup of $H / N$, then $P \leqslant \operatorname{Out}\left(\mathrm{L}_{2}\left(q^{2}\right)\right) \times \operatorname{Out}\left(\mathrm{L}_{2}\left(q^{2}\right)\right)$. Thus, $P$ is abelian, and it follows that $H / N$ is a subgroup of $A_{4} \times Z_{f}$ if $p$ is odd or $A_{3} \times Z_{f}$ if $p=2$ (containing a triality in either case). Thus, we have that $\delta_{H, N}(A) \leqslant 2$ with equality possible only if $|A|=p=2$, while $\delta_{H, H / N}(A) \leqslant 2$, with equality possible only if $A$ is central and $|A|=3$. This gives us what we need.

Finally, suppose that we are in case (viii) so that $q$ is odd. Our reference here is [30, Proposition 3.4.1]. We have that $H_{0}=N_{G_{0}}(P)$, where $P \leqslant G_{0}$ is elementary abelian of order 8 . Furthermore, $H_{0}$ can be realized as a subgroup of $\mathrm{P} \Omega_{8}^{+}(3)$. By using Magma, we can see that $H_{0}$ has three chief factors of order $2^{3}$, two of which are not $G$-equivalent (since they do not centralize each other). Since $H / H_{0} \leqslant \mathrm{Sym}_{4} \times Z_{f}$, the result now follows as above.

For the remainder of the paper, we may therefore assume that $G_{0}$ is an exceptional simple group. By [35, Theorem 2], the possibilities for $H$ are as follows.

THEOREM 7.2. Let $G$ be a finite almost simple group having as socle $G_{0}$ an exceptional simple group. If $H$ is a maximal subgroup of $G$, then one of the following holds: 
(i) $H$ is a maximal parabolic subgroup of $G$.

(ii) $H$ is almost simple.

(iii) $H=N_{G}(D)$ is the normalizer of a connected reductive $\sigma$-stable subgroup $D$ of $\boldsymbol{G}$ of maximal rank.

(iv) $H=N_{G}(E)$, where $E$ is an elementary abelian group.

(v) $F^{*}(H)$ is as in [35, Table III].

(vi) $H$ is the centralizer of a graph, field or graph-field automorphism of $G_{0}$ of prime order.

(vii) $G_{0}=E_{8}(q)$ and $F^{*}(H) \in\left\{\mathrm{Alt}_{5} \times \mathrm{Alt}_{6}, \mathrm{Alt}_{5} \times \mathrm{L}_{2}(q)\right\}$.

If $H$ is as in Theorem 7.2(ii), then the bounds in the conclusion of Theorem 2.7 follow immediately from Proposition 3.12. If $H$ is as in case (vii), then $H / F^{*}(H)$ is cyclic by [35, Theorem 2], so $\delta_{H}(A) \leqslant 1$ for all chief factors $A$ of $H$. Thus, we need only address cases (i), (iii), (iv), (v) and (vi).

We begin with the case where $H$ is as in Theorem 7.2(i).

Proposition 7.3. Let $G$ be an almost simple group with exceptional socle $G_{0}$ and let $H$ be a maximal parabolic subgroup of $G$. Then Theorem 2.7 holds.

Proof. Fix a non-Frattini chief factor $A$ of $H$. Since $H$ is maximal, $H$ is one of the following types:

(a) $H_{0}=P_{i}$ is the parabolic subgroup obtained by deleting node $i$ from the Dynkin diagram for $\mathbf{G}$;

(b) $G_{0}$ is either of type $F_{4}$ with $p=2$; type $B_{2}$ with $p=2$; type $G_{2}$ with $p=$ 3 ; or type $E_{6}$, and $G$ is either twisted or $G$ is untwisted and $G$ contains a graph or graph-field automorphism. Then $H_{0}=P_{i j}$ is the parabolic subgroup obtained by deleting nodes $i$ and $j$ from the Dynkin diagram for $\mathbf{G}$, where $\tau$ acts on the Dynkin diagram by interchanging nodes $i$ and $j$; or

(c) $G_{0}={ }^{3} D_{4}(q)$ and $H_{0}=P_{i j k}$ is the parabolic subgroup obtained by deleting nodes $i, j$ and $k$ from the Dynkin diagram for $\mathbf{G}$. The isometry $\tau$ acts as a 3 -cycle on the set $\{i, j, k\}$.

As in the proof of Proposition 6.6, we have $H_{0}=C \rtimes L$, where $C$ is the unipotent radical of $H_{0}$ and $L$ is a Levi subgroup. Since $C$ is characteristic in 
$H_{0} \unlhd H$, both $C$ and $C^{\prime}$ are normal subgroups of $H$. Thus, we have a normal series

$$
1 \leqslant C^{\prime} \leqslant C \leqslant C \rtimes L=H_{0} \leqslant H .
$$

Our strategy will be to investigate the structure of the factors in this series and then apply Lemma 3.4. Note that the group $C$ is a $p$-group, so $C^{\prime} \leqslant \operatorname{Frat}(C) \leqslant$ $\operatorname{Frat}(H)$.

We first consider the structure of $C$. Assume that we are in case (a) so that $H_{0}=P_{i}$. Here, the group $C / C^{\prime}$ is an irreducible module for $L$ over the field $\mathbb{F}_{q}$ of definition for $G_{0}$, by [5, Theorem 2(a)]. Hence, the group $C / C^{\prime}$ is a chief factor of $H$, of $p$-power order.

Assume now that we are in case (b). Then it follows from [5] that $C / C^{\prime}$ is either irreducible as an $L$-module or has two nonisomorphic irreducible composition factors. In the latter case, we have a series $1 \leqslant C^{\prime}<C_{1}<C$ for $C$, where each term is normalized by $L$. Moreover, $C_{1} / C^{\prime}$ and $C / C_{1}$ are nonisomorphic as $L$ modules, and hence nonisomorphic as $H_{0}=C \rtimes L$ modules. Since $C / C^{\prime}$ is an $H$-module and $H_{0} \unlhd H$, it follows from Clifford's theorem that either $1 \leqslant C^{\prime}<$ $C_{1}<C$ is also an $H$-series (in which case, $C_{1} / C^{\prime}$ and $C / C_{1}$ are irreducible $H$ modules) or $C / C^{\prime}$ is irreducible. Either way, we conclude that

$$
1 \leqslant C^{\prime} \leqslant C_{1} \leqslant C \leqslant C \rtimes L=H_{0} \leqslant H
$$

is a normal series for $H$, with $C^{\prime} \leqslant \operatorname{Frat}(H), C_{1} / C^{\prime}$ a chief factor of $H$ and $C / C_{1}$ either trivial or a chief factor of $H$ which is not $H$-equivalent to $C_{1} / C^{\prime}$.

Next, assume that we are in case (c) so that $G_{0}={ }^{3} D_{4}(q)$ and $H_{0}=P_{134}$. In this case, $C / C^{\prime}$ is an irreducible $H_{0}$-module of dimension 6 over $\mathbb{F}_{q}$. Thus, $C / C^{\prime}$ is an $H$-chief factor of order $q^{6}$.

We now consider the structure of $L$. Set $J:=\{1, \ldots, r\}-\{i\}$ if $H_{0}=P_{i}$, $J:=\{1, \ldots, r\}-\{i, j\}$ if $H_{0}=P_{i j}$ and $J:=\{1, \ldots, r\}-\{i, j, k\}$ if $H_{0}=P_{i j k}$. By [26, Theorem 2.6.5], we have $L=T M$, where $T$ is a maximal torus in $G_{0}$. Furthermore, $M=O^{p^{\prime}}(G), M=M_{1} \circ \cdots \circ M_{t}$ is a central product of finite groups of Lie type in characteristic $p$, and the elements of $T$ induce diagonal automorphisms on the components $M_{i}$ of $M$. By inspecting the Dynkin diagram of $\mathbf{G}$ in each case, one can see that the groups $M_{i} / Z\left(M_{i}\right)$ are either pairwise nonisomorphic or two of them are isomorphic to $\mathrm{L}_{2}\left(q^{2 u+1}\right)$. Furthermore, either $M_{i} / Z\left(M_{i}\right)$ is simple or $q \leqslant 3$ and $M_{i} / Z\left(M_{i}\right) \cong \mathrm{L}_{2}(q)$. For each $i$, define $L_{i}$ to be the product of those $M_{j}$ with $M_{j} H$-conjugate to $M_{i}$. Then $L_{i}$ is either a finite group of Lie type or isomorphic to a central product of $M_{i}$ and $M_{i}^{g}$, where $g \in H$ and $M_{i} / Z\left(M_{i}\right) \cong \mathrm{L}_{2}(q)$. Since $M C / C \unlhd H / C$, each group $L_{i} C / C$ is, in fact, normal in $H / C$. Since the distinct factors $L_{i} C / C$ of $M C / C \cong M$ have pairwise distinct centralizers in $M C / C$, and hence in $H / C$, it follows that if $A$ is a chief 
factor of $H / C$ contained in $M C / C$, then $A$ is contained in $L_{i} C / C$ for some $i$, and $\delta_{H, M C / C}(A)=\delta_{L_{i}}(A)$. Since $\delta_{X}(A)=1$ for all chief factors $A$ of $X$ for $X$ in $\left\{\mathrm{SL}_{2}(2), \mathrm{SL}_{2}(3)\right\}$, we deduce that if $L_{i} C / C=M_{i} C / C$, then $\delta_{H, M C / C}(A) \leqslant 1$ for all chief factors $A$ of $H$ contained in $L_{i} C / C$. Furthermore, equality holds only if $A$ is noncentral or if $M_{i} / Z\left(M_{i}\right)=\mathrm{L}_{2}(2)$ and $|A|=2$. If $L_{i} / Z\left(L_{i}\right) \cong \mathrm{L}_{2}(q)^{2}$, then since the two factors are permuted transitively by $H$, we again get $\delta_{H, M C / C}(A) \leqslant 1$ for all non-Frattini chief factors $A$ of $H$ contained in $L_{i} C / C$ (the chief factors in $Z\left(L_{i}\right)$ are Frattini).

We now consider $H / M C$. Using the definition of $M$ from [26, Theorem 2.6.5], we see that a field automorphism of $G_{0}$ acts as a field automorphism on each component $M_{i}$ of $M$. Similarly, if $H$ contains a graph automorphism $g$, then that graph automorphism must either centralize or act as a graph automorphism on $M_{i}$ if $L_{i}=M_{i}$. If $L_{i} \neq M_{i}$, then $g$ must swap the two components of $L_{i}$. Since $T$ induces diagonal automorphisms on $M_{i}$, we conclude that if $L_{i}=M_{i}$, then $H /(M C) \cong K . R$, where $K$ is a metacyclic subgroup of $\operatorname{Out}\left(M_{i} / Z\left(M_{i}\right)\right)$, and $|R| \leqslant 2$. Otherwise, $H /(M C) \cong K\langle g\rangle$, where $K=K_{1} K_{1}^{g}$ with $K_{1}$ a metacyclic subgroup of $\operatorname{Out}\left(M_{i} / Z\left(M_{i}\right)\right)$.

Next, we consider the chief factors of $H$ contained in the $p$-group $C$. The dimensions over $\mathbb{F}_{p}$ of the composition factors of the $\mathbb{F}_{p}[H]$-module $C / C^{\prime}$ are greater than 2 by [5, Theorem 2]. In particular, $\delta_{H, C / C^{\prime}}(A) \leqslant 1$, and $\delta_{H, C}(A)=0$ if either $A$ is not a $p$-group or $A$ is a $p$-group with $\operatorname{dim}_{\mathbb{F}_{p}}(A) \leqslant 2$.

We are now ready to complete the proof of the proposition. By the arguments above, we have that $H$ has shape C.M.K.R, where $C, M, K$ and $R$ are as above. If $A$ is contained in $C$, then $A$ is either Frattini or $A$ is contained in $C / C^{\prime}$. In the latter case, since none of the groups $M, K$ or $R$ have chief factors of dimension larger than 1 , we have $\delta_{H}(A)=1$ by the previous paragraph. Suppose next that $A$ is contained in $M$. Then we have $\delta_{H, M C / C}(A)=1$. If $A$ is nonabelian, then $\delta_{H}(A)=1$ since $H / M C$ is soluble. If $A$ is abelian and non-Frattini, then we must have $q \leqslant 3$ and $L_{i} / Z\left(L_{i}\right) \cong \mathrm{L}_{2}(q)^{s}$ for some $i$ and some $s \leqslant 2$. In this case, since $T$ induces diagonal automorphisms on $M_{i} / Z\left(M_{i}\right)$, we have $H /\left(C \hat{L}_{i}\right) \leqslant \mathrm{GL}_{2}(q) \times$ $Z_{2}$ if $s=1$ and $H /\left(C \hat{L}_{i}\right) \leqslant \mathrm{GL}_{2}(q) \geq Z_{2}$ if $s=2$, where $\hat{L}_{i}$ denotes the product of all components of $M$ not equal to $M_{i}$. We then get $\delta_{H}(A)=\delta_{H /\left(C \hat{L}_{i}\right)}(A) \leqslant 2$, which gives us what we need. Finally, suppose that $A$ is in $K . R$ but not in C.M. Then since $K$ is metacyclic, we have $\delta_{H}(A) \leqslant 3$, with equality possible only if $|A|=2$. This completes the proof.

Next, we consider the case where $H$ is as in Theorem 7.2(iii).

Proposition 7.4. Let $G$ be an almost simple group with exceptional socle $G_{0}$ and let $H$ be a maximal subgroup of $G$ as in Theorem 7.2(iii), where $D$ is not a torus. Then Theorem 2.7 holds. 
Proof. Fix a non-Frattini chief factor $A$ of $H$. Here, the group $D$ is defined as follows: let $\Delta$ be a closed subset of $\Phi$ maximal with the property that $\Delta$ is a root system, and the set of rays through elements of $\Delta$ is $\tau$-invariant (see [26, Definition 2.3.1]). Then define

$$
D_{\Delta}:=\left\langle\hat{U}_{\alpha}: \alpha \in \Delta\right\rangle,
$$

where $\hat{U}_{\alpha}$ denotes the root subgroups in $\mathbf{G}$ with respect to a maximal torus $T=T_{\Delta}$ in G. Recall that $W$ is the Weyl group of G. Let $W(\Delta)$ be the Weyl group of $D_{\Delta}$, and let $\operatorname{Stab}_{W}(\Delta)$ denote the stabilizer of $\Delta$ in $W$ so that $\operatorname{Stab}_{W}(\Delta)$ normalizes $W(\Delta)$. For any $w \in \operatorname{Stab}_{W}(\Delta)$, define a subgroup of $\mathbf{G}$ as follows. Choose $n \in$ $N_{\mathbf{G}}(T)$ mapping to $w \in W \cong N_{\mathbf{G}}(T) / T$, and choose $x \in \mathbf{G}$ such that $\sigma=(\sigma n)^{x}$ (see, for example, [26, Theorem 2.1.2(f)]). Then set

$$
D_{\Delta, w}:=D_{\Delta}^{x} T^{x} .
$$

The group $D$ is defined to be one of the $D_{\Delta, w}$ as above.

Now, retaining the notation above, set $M:=D_{\Delta}^{x}$ and $S:=T^{x}$. Also, set $W_{\Delta}:=\operatorname{Stab}_{W}(\Delta) / W(\Delta)$. Then $D_{\sigma}=M_{\sigma} S_{\sigma}$, and $N_{I}(D)=N_{I}\left(D_{\sigma}\right)=$ $D_{\sigma} \cdot C_{W_{\Delta}}(W(\Delta) w)=\left(M_{\sigma} S_{\sigma}\right) . W_{\Delta}$, by [26, Proposition 2.6.2]. In particular, since $\operatorname{Stab}_{W}(\Delta) \leqslant W$ acts faithfully on the maximal torus $S \leqslant D$, we have that $W_{\Delta}$ acts faithfully on $D$. Hence, $C_{W_{\Delta}}(W(\Delta) w)$ acts faithfully on $S$, and since $N_{I}\left(D_{\sigma}\right) / D_{\sigma} \cong N_{\mathbf{G}}(D) / D=W_{\Delta}$, it follows that $C_{I}\left(D_{\sigma}\right) \leqslant D_{\sigma}$.

The structure of $N:=N_{I}(D)$ is given in [34, Table 5.1]. Note that $N_{I^{\prime}}(D) \leqslant$ $H_{I}:=H \cap I \leqslant N$, and $N / N_{I^{\prime}}(D)$ is cyclic of order dividing $\left|I / I^{\prime}\right|$, the group of diagonal automorphisms of $G_{0}$. Thus,

$$
H \cap N \unlhd N \quad \text { and } \quad N / H \cap N \text { has order dividing }\left|I / I^{\prime}\right| .
$$

We now proceed to examine each of the cases in [34, Table 5.1]. Recall that we are trying to prove that

$$
\delta_{H}(A) \leqslant 3 \text { if } A \text { is abelian, and } \delta_{H}(A) \leqslant 2 \text { if } A \text { is nonabelian. }
$$

Using the bound $\delta_{H}(A) \leqslant \delta_{H, H_{I}}(A)+\delta_{H / H_{I}}(A)$ and appealing to Table 2 for the chief factors of $H / H_{I}$, the result is clear in most cases. For example, consider $G_{0}={ }^{3} D_{4}(q)$. One of the two possibilities for $H_{I}$ in [34, Table 5.1] has shape $K$. $a$, where $a$ divides $(2, q-1)$, and $K$ has shape $Z$. $\left(\mathrm{L}_{2}(q) \circ \mathrm{L}_{2}\left(q^{3}\right)\right)$, where $Z$ is Frattini in $K$ (and hence Frattini in $H$, since $K$ is subnormal in $H$ ). We then get $\delta_{H}(A) \leqslant 2$ for any $A$ since $H / H_{I}$ is cyclic (see Table 2). This approach works in almost all cases, except for the following:

(a) $G_{0}=E_{6}^{\epsilon}(q)$ and $N=E$. $\operatorname{Sym}_{3}$, where $E=J \circ R$, with $J \cong \Omega_{8}^{+}(q)$ and $R=(q-\epsilon)^{2}$. 
(b) $G_{0}=E_{6}^{\epsilon}(q)$ and $N=J$. $\operatorname{Sym}_{3}$, where $J=e .\left(\mathrm{L}_{3}^{\epsilon}(q) \times \mathrm{L}_{3}^{\epsilon}(q) \times \mathrm{L}_{3}^{\epsilon}(q)\right) \cdot e^{2}$ and $e:=(3, q+\epsilon)$.

(c) $G_{0}=E_{7}(q)$ and $N=\left(J_{1} \circ J_{2}\right) \cdot d^{3} \cdot \operatorname{Sym}_{3}$, where $J_{1}=\mathrm{SL}_{2}(q)^{3}, J_{2}=\Omega_{8}^{+}(q)$ and $d:=(2, q-1)$.

(d) $G_{0}=E_{7}(q)$ and $N=J \cdot \mathrm{L}_{3}(2)$, where $J=d^{3} \cdot \mathrm{L}_{2}(q)^{7} \cdot d^{4}$ and $d:=(2, q-1)$.

(e) $G_{0}=E_{8}(q)$ and $N=J .\left(\operatorname{Sym}_{3} \times 2\right)$, where $J=d^{2} \cdot \mathrm{P} \Omega_{8}^{+}(q)^{2} \cdot d^{2}$ and $d:=(2$, $q-1)$.

(f) $G_{0}=E_{8}(q)$ and $N=J$. GL $2(3)$, where $J=e^{2} \cdot \mathrm{L}_{3}^{\epsilon}(q)^{4} \cdot e^{2}$ and $e:=(3, q-\epsilon)$.

(g) $G_{0}=E_{8}(q)$ and $N=J \cdot \operatorname{AGL}_{3}(2)$, where $J=d^{4} \cdot \mathrm{L}_{2}(q)^{8} \cdot d^{4}$ and $d:=(2$, $q-1)$.

In the notation introduced in the first paragraph above, we are defining $E:=$ $D_{\sigma}, J:=M_{\sigma}$ and $R:=S_{\sigma}$. Also, $W_{\Delta}$ is $\operatorname{Sym}_{3}, \operatorname{Sym}_{3}, \operatorname{Sym}_{3}, \mathrm{~L}_{3}(2), \operatorname{Sym}_{3} \times 2$, $\mathrm{GL}_{2}(3)$ and $\mathrm{AGL}_{3}(2)$ in these cases, respectively. By the arguments above, we have $C_{G}(E) \leqslant E$.

We now deal with these outstanding cases. Suppose first that $G_{0}=E_{6}^{\epsilon}(q)$ so that $H / H_{I}$ is a subgroup of $Z_{f} \times Z_{2}$ (see Table 2) and that $N=E . S_{3}$, where $E=J \circ R$, with $J \cong \Omega_{8}^{+}(q)$ and $R=(q-\epsilon)^{2}$. Since there is a unique non-Frattini chief factor in $J$, which is nonabelian, and this is the only nonabelian chief factor of $H$, we have $\delta_{H}(A)=1$ if $A$ is nonabelian, and $\delta_{H}(A)=\delta_{H / J}(A)$ otherwise.

So we need to consider the chief factors in the soluble group $H / J$. We first determine the action of $N$ on $R$. Without loss of generality, we will take $\Phi$ to be as in [8, Page 260], with set of fundamental roots $\Pi:=\left\{\alpha_{1}, \ldots, \alpha_{6}\right\}$. The longest root $\alpha_{0}$ is defined by $\alpha_{0}:=\alpha_{1}+2 \alpha_{2}+2 \alpha_{3}+3 \alpha_{4}+2 \alpha_{5}+\alpha_{6}$. From this, we may form the extended Dynkin diagram of type $E_{6}$ with vertices $\widetilde{\Pi}:=\left\{-\alpha_{0}, \alpha_{1}, \ldots\right.$, $\left.\alpha_{6}\right\}$. Denote by $X=X(T)$ the set of rational characters of $T$.

Now, without loss of generality, we can take $\Delta$ to be the $D_{4}$-subsystem of $E_{6}$ with fundamental roots $\Pi_{D}=\left\{\alpha_{2}, \alpha_{3}, \alpha_{4}, \alpha_{5}\right\}$. Let $X_{D}$ be the subspace of $X \otimes \mathbb{Q}$ generated by $\Delta$. Also, let $V:=X \otimes \mathbb{Q} / X_{D}$. Then $\left\{\alpha_{1}, \alpha_{6}\right\}$ reduces, modulo $X_{D}$, to a basis for $V$. Using Magma, one can see that $\operatorname{Stab}_{W}(\Delta) \cap \operatorname{Stab}_{W}(\widetilde{\Pi})$ contains an element $a$ whose induced action on $\widetilde{\Pi}$ is $\left(\alpha_{2}, \alpha_{3}, \alpha_{5}\right)\left(\alpha_{1}, \alpha_{6},-\alpha_{0}\right)$. Similarly, $\operatorname{Stab}_{W}(\Delta)$ contains an element $b$ which acts on $\Delta$ as $\left(\alpha_{3}, \alpha_{5}\right)$ (but this element does not stabilize $\widetilde{\Pi})$. Furthermore, $\alpha_{1}^{b}=\alpha_{6}$ modulo $X_{D}$. Since $\alpha_{0}=\alpha_{1}+\alpha_{6}$ modulo $X_{D}$, it follows that the induced action of $W_{\Delta}$ on $A$ is

$$
W_{\Delta}^{V}=\left\langle\left[\begin{array}{cc}
-1 & -1 \\
1 & 0
\end{array}\right],\left[\begin{array}{ll}
0 & 1 \\
1 & 0
\end{array}\right]\right\rangle \cong \operatorname{Sym}_{3} .
$$


Now, recall that the action of $\sigma$ on $X(T)$ commutes with the action of $W$ on $X(T)$. By [12, Proposition 7 and the discussion following], $V /(\sigma-1) V$ is isomorphic as a $W_{\Delta}$-group to $S_{\sigma}=R$. Hence, since $R \cong(q-\epsilon)^{2}$, we may write

$$
V /(\sigma-1) V=\langle\chi\rangle \times\langle\Sigma\rangle \cong(q-\epsilon)^{2} .
$$

We have $\chi^{a}=\chi^{-1} \Sigma^{-1}$ and $\chi^{b}=\Sigma$ by (7.5). In particular, it follows that for any prime $p_{1}$ dividing $q-1, W_{\Delta}=\langle a, b\rangle \cong \mathrm{Sym}_{3}$ acts irreducibly on

$$
Y_{p_{1}}:=\left[S_{\sigma} / O^{p_{1}}\left(S_{\sigma}\right)\right] /\left[\operatorname{Frat}\left(S_{\sigma} / O^{p_{1}}\left(S_{\sigma}\right)\right)\right] \cong p_{1}^{2} .
$$

Furthermore, $Y_{2} \cdot\langle a\rangle \cong \mathrm{Alt}_{4}$ and $Y_{2} \cdot\langle b\rangle \cong D_{8}$.

With the information deduced above, we can now determine the required upper bounds on $\delta_{H}(A)$, for each non-Frattini chief factor $A$ of $H$. We have a normal series

$$
1<J \leqslant H \cap D_{\sigma} \leqslant H \cap N \leqslant H \leqslant(H \cap N) \cdot\langle\phi\rangle \cdot\langle g\rangle,
$$

where $\phi$ represents a field automorphism of $G_{0}$ and $g$ is a graph automorphism. The field and graph automorphisms normalize $D_{\sigma}$, by [34, Lemma 1.3].

As mentioned at the beginning of our analysis of this case, we need only consider the $H$-chief factors contained in $H \cap D_{\sigma} / M_{\sigma} \leqslant S_{\sigma} / Z$. Indeed, by the arguments in the paragraph above, they can be completely determined by the group $H \cap N / H \cap D_{\sigma}$. Since $N / D_{\sigma} \cong W_{\Delta} \cong \operatorname{Sym}_{3}$ (see [34]) and $|N / H \cap N|$ divides 3 by (7.3), this group has order 2 or 6 . If it has order 2, then $H \cap N$ acts on $S_{\sigma}$ by switching the cyclic factors, so we get at most two chief factors of order $l$ for each prime $l$ dividing $q-\epsilon$, except that one of these is Frattini in the case $l=2$. Otherwise, we get at most one non-Frattini $H$-chief factor in $S_{\sigma} / Z$ for each prime dividing $q-\epsilon$. All of this information follows from the arguments above.

Since $\sigma$ and $\phi$ commute, it now follows that $\delta_{H}(A) \leqslant 3$ for all $A$, with equality possible only if $|A|=2$. This gives us what we need.

We now consider the cases (b)-(g). In each case, we have $S_{\sigma}=1$ and $D_{\sigma}^{\prime}=$ $M_{\sigma}^{\prime}=J^{\prime}$ is a central product $J_{1} \circ J_{2}$ of normal subgroups $J_{i}$ of $N$, where $J_{i}$ is either trivial or a central product $M_{1} \circ M_{2} \circ \cdots \circ M_{l}$, where the $M_{i}$ are pairwise isomorphic, are normalized by $D_{\sigma}$ and each $M_{i}$ is either quasisimple or in $\left\{\mathrm{SL}_{2}(2), \mathrm{SL}_{2}(3)\right\}$. By [26, Proposition 2.6.2c], $D_{\sigma} / D_{\sigma}^{\prime}$ induces diagonal automorphisms on each $M_{i}$. Also, since $C_{I}\left(D_{\sigma}\right) \leqslant D_{\sigma}$, we have that $N / D_{\sigma}^{\prime} \leqslant \operatorname{Out}\left(D_{\sigma}^{\prime}\right)$. The result now follows easily. For example, suppose that we are in case (b) above. Then $G_{0}=$ $E_{6}^{\epsilon}(q)$ and $N=J . \operatorname{Sym}_{3}$, where $J=e .\left(\mathrm{L}_{3}(q) \times \mathrm{L}_{3}(q) \times \mathrm{L}_{3}(q)\right) . e^{2}$, and $e:=(3$, $q+\epsilon)$. We have $\operatorname{Out}\left(J^{\prime}\right) \cong \operatorname{Out}\left(\mathrm{L}_{3}(q)\right)$ 2 $\operatorname{Sym}_{3}$. Hence, since the $e^{2}$ on top of $J$ induces diagonal automorphisms of $\mathrm{L}_{3}(q)$, we have $N / J \leqslant Z_{3}$ 2 $\operatorname{Sym}_{3}$. In fact, if $e>1$, then it follows from Lemma 3.9 that $N / J=Y \rtimes \operatorname{Sym}_{3}$, where $Y$ is the fully deleted permutation module of $\operatorname{Sym}_{3}$. Since $H_{I} / J$ has index at most 3 in 
$N / J$, we deduce that if $e>1$, then the $e^{2}$ on the top of $J$ either consists of two noncentral chief factors if $\left|H_{I} / J\right|=2$ or one noncentral chief factor otherwise. Furthermore, at least two of the factors in the section $\mathrm{L}_{3}(q)^{3}$ are conjugate in $H$. Hence, $\delta_{H}(A)=1$ if $A$ is nonabelian. Since $H / H_{I}$ is abelian, we also deduce that $\delta_{H}(A) \leqslant 2$ for any noncentral non-Frattini chief factor of $A$. Finally, $Z(J) \cong Z_{3}$ is Frattini and $H / H_{I}$ is metacyclic, so we have $\delta_{H}(A) \leqslant 3$ if $A$ is abelian (with equality possible only if $|A|=2$ and $f$ is even).

As another example, consider case $(\mathrm{g})$ so that $G_{0}=E_{8}(q)$ and $N=J$. $\mathrm{AGL}_{3}(2)$, where $J=d^{4} \cdot \mathrm{L}_{2}(q)^{8} \cdot d^{4}$, and $d:=(2, q-1)$. As above, we have that $Z(J)=d^{4}$ is Frattini in $J$, and hence Frattini in $H$. Also, the $d^{4}$ on top of $J$ induces diagonal automorphisms in the factors of the section of $\mathrm{L}_{2}(q)^{8}$. Furthermore, since $N / J^{\prime} \leqslant \operatorname{Out}\left(J^{\prime}\right)$ and $H_{I} / J^{\prime} \cong d^{4}$. $\operatorname{AGL}_{3}(2)$, we deduce that $H_{I} / J \cong \operatorname{AGL}_{3}(2)$ and $H_{I} / J^{\prime} \leqslant Z_{2} . Z_{3} 2 \mathrm{GL}_{3}(2)$. Also, the intersection of $H_{I} / J^{\prime}$ with the base group of this wreath product has order $d^{4} .3^{2}$. This is because the only quotient $\mathrm{AGL}_{3}(2) / K$ of the affine group with soluble $K$ and an embedding $\mathrm{AGL}_{3}(2) / K \rightarrow$ $\mathrm{Sym}_{8}$ is the group $\mathrm{GL}_{3}(2)$ acting on the nonzero vectors of its natural module. We can now determine $\delta_{H}(A)$ for each chief factor $A$ of $H$. We have

$$
H=d^{4} \cdot \mathrm{L}_{2}(q)^{8} \cdot d^{4} \cdot 3^{2} \cdot \mathrm{GL}_{3}(2) \cdot Z,
$$

where $Z \leqslant Z_{f}$. The $d^{4}$ at the bottom is Frattini. If $L_{2}(q)$ is simple, then the section $\mathrm{L}_{2}(q)^{8}$ is a chief factor since $\mathrm{GL}_{3}(2)$ permutes the copies transitively. If $q \leqslant 3$, then one can find the non-Frattini chief factors of $H$ contained in $\mathrm{L}_{2}(q)^{8}$ by direct computation. If $q=2$, they have orders $3^{8}, 2$; while if $q=3$, they have orders $2^{16}, 3^{7}, 3$.

Next, one can also quickly check in Magma (using the PermutationModule function) that the submodules of the (indecomposable) $\mathrm{GL}_{3}(2)$-permutation module $2^{8}$ have dimensions $0,1,4,7$ and 8 . Whence, if $d>1$, then the $2^{4}$ on top of $J$ is an $H$-module of composition length 2, consisting of a (necessarily Frattini) submodule of dimension 1 and a noncentral chief factor of dimension 3 . The $3^{2}$ is also a noncentral chief factor of $H$, and $\mathrm{GL}_{3}(2)$ is a nonabelian chief factor. Finally, $Z$ is a central factor. The required bounds now follow in each case.

Proposition 7.5. Let $G$ be an almost simple group with exceptional socle $G_{0}$ and let $H$ be a maximal subgroup of $G$ as in Theorem 7.2(iii), where $D$ is a torus. Then Theorem 2.7 holds.

Proof. Fix a non-Frattini chief factor $A$ of $H$. Here we have $H=N_{G}\left(T_{\sigma}\right)$, where $T$ is a $\sigma$-stable maximal torus in $\mathbf{G}$. Furthermore, the groups $T_{\sigma}$ and $N_{\mathbf{G}_{\sigma}}\left(T_{\sigma}\right) / T_{\sigma}$ are given in [34, Table 5.2]. The latter is, in fact, a subgroup $C$ of the Weyl group $W$ of $\mathbf{G}$ (see [26, Theorem 2.3.4]), and our strategy will be to determine the action 
of $C$ on the abelian group $T_{\sigma}$ : from this, we can completely determine the nonFrattini chief factors of $N_{\mathbf{G}_{\sigma}}\left(T_{\sigma}\right)$. We will frequently use the fact that

$$
H \cap I \geqslant N_{G_{0}}\left(T_{\sigma}\right)=N_{\mathbf{G}_{\sigma}}\left(T_{\sigma}\right) T_{\sigma}
$$

to deduce facts about the non-Frattini $H$-chief factors contained in $H \cap I$.

The abelian group $T_{\sigma}$ is the direct product of its $l$-parts (for $l$ prime), so the non-Frattini chief factors will be determined by the $\mathbb{F}_{l}[C]$-modules $M_{l}:=$ $\left(T_{\sigma}\right)_{l} / \operatorname{Frat}\left(\left(T_{\sigma}\right)_{l}\right)$. If $l$ does not divide $|C|$, then $M_{l}$ lifts to a $\overline{\mathbb{Q}}[C]$-module $\overline{M_{l}}$, and the dimensions of the composition factors of $M_{l}$ are the same as those for $\overline{M_{l}}$. In the finitely many cases, where $l$ divides $|C|$, we can use Magma to find the composition factors of $M_{l}$ as a $\mathbb{F}_{l}[C]$-module (see Remark 7.6). Apart from two cases, we find that $M_{l}$ is irreducible as an $\mathbb{F}_{l}[C]$-module, and hence as an $\mathbb{F}_{l}[H]$-module, by (7.6). In most cases, $\operatorname{dim} M_{l}$ is large, so one can immediately deduce that $\delta_{H}\left(M_{l}\right)=1$ for all $l$. In general, we can quickly deduce from Table 2 and [34, Table 5.2] that $\delta_{H}\left(M_{l}\right) \leqslant 2$ if $\left|M_{l}\right|>2$ and $\delta_{H}\left(M_{l}\right) \leqslant 3$ if $\left|M_{l}\right|=2$. The remaining chief factors of $H$ are all contained in $C$ and $H / H_{0}$, and we can deduce the bound for $\delta_{H}(A)$ immediately from Table 2 and [34, Table 5.2] again.

This leaves us with the cases mentioned above where $M_{l}$ is reducible as an $\mathbb{F}_{l}[C]$-module. By the arguments above, we may assume that $M_{l}$ is reducible as an $H$-module as well. These cases are as follows:

(i) $G_{0}=E_{6}^{\epsilon}(q), 3$ divides $q-\epsilon, T_{\sigma}=(q-\epsilon)^{6}$ and $l=3$. In this case, $M_{l}$ has two $C$-composition factors $A_{l}$ and $B_{l}$ of dimensions 1 and 5, respectively. Since $M_{l}$ is also reducible as an $H$-module, we deduce from (7.6) that $A_{l}$ and $B_{l}$ are also $H$-composition factors. Since $C=W\left(E_{6}\right)=S .2$, where $S$ is nonabelian simple and $H / H_{0}$ has at most two non-Frattini chief factors of order 3, at least one of which must be central, the required bound follows for $A=B_{l}$. Clearly, $\delta_{H}\left(A_{l}\right)=1$, and so we have what we need.

(ii) $G_{0}=E_{7}(q), q$ is odd, $T_{\sigma}=(q-\epsilon)^{7}$ with $\epsilon= \pm$ and $l=2$. Here $M_{l}$ has two $C$-composition factors of dimensions 1 and 6 , and the argument follows as above.

REMARK 7.6. We remark that the subgroup $C$ from the proof of Proposition 7.5 is the centralizer of $\sigma w$, where $w$ is an element in the Weyl group of $G$. We now briefly describe how to use Magma to compute the composition factors of the group $T_{\sigma}$ as in the proof above. First, use the Magma function WeylGroup to compute $W$. Then we convert $W$ to a matrix group over $\mathbb{Q}$ by using Reflectiongroup (W). One can then compute the possibilities for $C$, before converting $C$ to a matrix group over $\mathbb{F}_{l}$ (if $l$ divides $|C|$ ) using 
the function $\operatorname{sub}<\mathrm{GL}(r, 1) \mid$ Generators $(C)>$. We then use the function Compositionfactors (GModule (C)) to compute the composition factors of the natural $C$-module, where $\mathbb{F}:=\mathbb{F}_{l}$ or $\mathbb{F}:=\mathbb{Q}$ according to whether $l$ divides $|C|$ or not, respectively. For example, if $G=E_{6}(q), l=3$ and $T_{\sigma}$ has Cartan diagram of type $3 A_{2}$ (see [34, Table 5.2]), then $C$ is the centralizer of the seventh representative in the list of conjugacy classes of $W$ stored in Magma, and our code reads as follows:

$\mathrm{G}:=$ GroupofLieType("E6", RationalField());

$\mathrm{W}:=$ WeylGroup $(\mathrm{G})$;

$\mathrm{R}:=$ ReflectionGroup $(\mathrm{W}) ; \mathrm{CC}:=$ ConjugacyClasses $(\mathrm{R})$;

$\mathrm{C}:=$ Centraliser (R, CC [7] [3] ); h:=Generators (C);

$\mathrm{C} 1:=$ sub $<\mathrm{GL}(6,3) \mid \mathrm{h}>$; ; V:=GModule (C1) ;

Compositionseries (V);

Proposition 7.7. Let $G$ be an almost simple group with exceptional socle $G_{0}$ and let $H$ be a maximal subgroup of $G$ as in Theorem 7.2(iv) or (v) above. Then Theorem 2.7 holds.

Proof. Fix a non-Frattini chief factor $A$ of $H$. The structure of the group $N_{\mathbf{G}_{\sigma}}(H)$ is given in [35, Table III] in case $(\mathrm{v})$, and the bounds on $\delta_{H}(A)$ follow easily in every case.

So assume that we are in case (iv). Then $H$ normalizes an elementary abelian subgroup $E$ of $\mathbf{G}_{\sigma}$, and the structure of $N_{\mathbf{G}_{\sigma}}(E)$ is given by [13, Theorem 1 part (II)]. In particular, $N_{\mathbf{G}_{\sigma}}(E) / C_{\mathbf{G}_{\sigma}}(E)$ acts irreducibly on $E$, so $\delta_{H, E}(E)=1$.

Suppose first that $\mathbf{G}=E_{8}$ (so that $I \leqslant G$ ), and $H \cap I=N_{\mathbf{G}_{\sigma}}(E)=$ $2^{5+10} . \mathrm{SL}_{5}(2)$. In this case, $E=2^{5}, N_{\mathbf{G}_{\sigma}}(E) / C_{\mathbf{G}_{\sigma}}(E)$ acts naturally on $E$, and $C_{\mathbf{G}_{\sigma}}(E)$ is special of order $2^{15}$. Since $\mathrm{SL}_{5}(2)$ is simple and $I / H \cap I$ is cyclic, we must have that $H \cap I=N_{\mathbf{G}_{\sigma}}(E)=2^{5+10} . \mathrm{SL}_{5}(2)$. In particular, $Z\left(C_{\mathbf{G}_{\sigma}}(E)\right)=E$, from which it follows that $\delta_{H, C_{\mathbf{G}_{\sigma}}(E)}(E)=1$. From the proof of [13, Theorem 1 part (II)], we can see that $C_{\mathbf{G}_{\sigma}}(E) / E=U \oplus U^{\prime} \cong 2^{5} \oplus 2^{5}$ as an $\mathrm{SL}_{5}$ (2)-module, with the $\mathrm{SL}_{5}(2)$ acting naturally on each of $U, U^{\prime}$. Clearly, we have $\delta_{H}(A) \leqslant 2$ for $A \in\left\{E, U, U^{\prime}\right\}$. Finally, since $\operatorname{Out}\left(G_{0}\right)$ is cyclic, we have $\delta_{H}(A) \leqslant 1$ for any other chief factor $A$ of $H$.

The same strategy as above works in the case $\mathbf{G}=E_{6}$ and $N_{\mathbf{G}_{\sigma}}(E)=$ $3^{3+3} \cdot \mathrm{SL}_{3}(3)$, except that in this case, we have $H \cap I=N_{\mathbf{G}_{\sigma}}(E)$ since $N_{\mathbf{G}_{\sigma}}(E)$ is perfect and $N_{\mathbf{G}_{\sigma}}(E) / H \cap I$ is cyclic.

Next, assume that $\mathbf{G}=E_{7}$ (so that $|I: H \cap I| \leqslant 2$ ), and $H \cap I \leqslant N_{\mathbf{G}_{\sigma}}(E)=$ $\left(2^{2} \times \operatorname{Inndiag}\left(\mathrm{P} \Omega_{8}^{+}(q)\right)\right) . \mathrm{Sym}_{3}$. Here, the $\mathrm{Sym}_{3}$ on top acts naturally as $\mathrm{GL}_{2}(2)$ on $E=2^{2}$, and $H \cap I / E \leqslant \operatorname{Aut}\left(\mathrm{P} \Omega_{8}^{+}(q)\right)$. Hence, we have $H \cap I=N_{\mathbf{G}_{\sigma}}(E)=\left(2^{2} \times\right.$ $\left.\operatorname{Inndiag}\left(\mathrm{P} \Omega_{8}^{+}(q)\right)\right) . \operatorname{Sym}_{3}$ or $H \cap I=N_{\mathbf{G}_{\sigma}}(E)=\left(2^{2} \times \operatorname{Inndiag}\left(\mathrm{P} \Omega_{8}^{+}(q)\right)\right)$. $\operatorname{Alt}_{3}$. 
Either way, we have $\delta_{H}(E)=2$. Furthermore, since $\operatorname{Out}\left(G_{0}\right)=Z_{(2, q-1)} \times Z_{f}$, we have $\delta_{H}\left(Z_{2}\right) \leqslant 3, \delta_{H}\left(\mathrm{P} \Omega_{8}^{+}(q)\right)=1$ and $\delta_{H}\left(Z_{3}\right) \leqslant 2$ if the action on $Z_{3}$ is trivial, and $\delta_{H}\left(Z_{3}\right)=1$ otherwise.

The remaining cases are easier and follow from the same arguments as above; in every case, we have $N_{\mathbf{G}_{\sigma}}(E)=p^{n} . S$, with $S \leqslant \mathrm{GL}_{n}(p)$ simple and irreducible. We then use Table 2 to bound the contribution of the outer automorphism group to $\delta_{H}(A)$ in each case, and this completes the proof.

Proposition 7.8. Let $G$ be an almost simple group with exceptional socle $G_{0}$ and let $H$ be a maximal subgroup of $G$ as in Theorem 7.2(vi). Then Theorem 2.7 holds.

Proof. If $p=2$, then the structure of $H_{0}$ is given in [4, Section 19], while if $p>2$, the structure of $H_{0}$ is given in [25, Section 7] and [13, Proposition 2.7]. In particular, $F^{*}\left(H_{0}\right)$ is quasisimple. Furthermore, it is clear in each case that an element of $H-F^{*}\left(H_{0}\right)$ either acts trivially on $F^{*}\left(H_{0}\right)$ or induces an outer automorphism of $H_{0}$.

In particular, $H / F^{*}\left(H_{0}\right)$ has shape D.E.F, where $D, E$ and $F$ are (possibly trivial) cyclic groups. Furthermore, using Table 2, we can see that at least one pair of groups from $\{D, E, F\}$ has coprime orders. Whence, $\delta_{H}(A) \leqslant 1$ if $A$ is nonabelian, and $\delta_{H}(A) \leqslant 3$ otherwise.

We conclude the paper by showing that there exist infinitely many pairs $(G, H)$ where $G$ is almost simple, $H$ is a maximal subgroup of $G, d(H)=3$ and $H$ does not have an elementary abelian quotient of order $l^{3}$, for any prime $l$. Thus, our theorem is 'best possible'.

EXAMPLE 7.9. Let $p$ be a prime such that $p \equiv-1(\bmod 3)$ and let $n=2 m$ with $m$ odd and 3 dividing $m$, and set $G$ to be the subgroup of $\operatorname{Aut}\left(\mathrm{L}_{n}\left(p^{2}\right)\right)$ generated by inner, diagonal and field automorphisms. Let $H$ be a maximal parabolic subgroup of $G$ in class $\mathcal{C}_{1}$, of type $P_{m}$. Let $C$ be the unipotent radical of $H$, and let $L / C^{\prime}$ be a complement in $H / C^{\prime}$ for $C / C^{\prime}$ so that $H=C L$ (such a complement exists since $C / C^{\prime}$ is a non-Frattini minimal normal subgroup of $H$ in this case). Then $L / C^{\prime}$ contains a characteristic subgroup $M / C^{\prime}$ which is a central product $M / C^{\prime} \cong \overline{\Omega_{1}} \circ \overline{\Omega_{2}}$ of almost simple groups $\overline{\Omega_{i}}$, with $\overline{\Omega_{i}} \cong \mathrm{L}_{m}\left(p^{2}\right)$ for $i=1,2$. Let $L_{I}$ (respectively $H_{I}$ ) be the intersection of $L$ (respectively $H$ ) with $\operatorname{PGL}_{n}\left(p^{2}\right) \leqslant$ Aut $\left(\mathrm{L}_{n}\left(p^{2}\right)\right)$. By [31, Lemma 4.1.1], we have $L_{I} / M \cong \overline{I_{1}} / \overline{\Omega_{1}} \times \overline{I_{2}} / \overline{\Omega_{2}}$, where $\overline{I_{i}} \cong \mathrm{PGL}_{m}\left(p^{2}\right)$ for each $i$. Whence, $H_{I} / C M \cong C L_{I} / C M \cong L_{I} / M$ is isomorphic to $E_{1} \times E_{2}$, where $E_{1} \cong E_{2} \cong Z_{d_{1}}$, with $d_{1}:=\left(m, p^{2}-1\right)$. In particular, it follows that $H / C M \cong\left(Z_{d_{1}} \times Z_{d_{1}}\right) . Z_{2}$, where the $Z_{2}$ on top is the group of field 
automorphisms of $\operatorname{soc}(G)$. By definition of the subgroup $H$, we have that $Z_{2}$ acts on each $\overline{\Omega_{i}}$ as a field automorphism. Thus, a generator $x$ of $Z_{2}$ acts on a generator $y_{i}$ of $E_{i}$ by $y_{i}^{x}=y_{i}^{p}$. In particular, since 3 divides $d_{1}$ and $p \equiv-1(\bmod 3)$, we deduce that $H$ has a factor group $H / R \cong 3^{2}: 2$, where the 2 acts by inverting the nonzero elements in the $3^{2}$. Hence, $d(H / R)=3$. We have $d(H) \leqslant 3$ by the main theorem. However, since $L$ is a central product of two quasisimple groups and $d_{1}$ and $p$ are odd, we have $\delta_{H}\left(Z_{2}\right)=\delta_{H / C L_{I}}\left(Z_{2}\right)=1$.

\section{Acknowledgements}

We would like to express our deep gratitude to the anonymous referee who reviewed our paper. Their time, effort and diligence, together with their excellent comments and suggestions, have improved this paper immeasurably. Research partially supported by MIUR-Italy via PRIN Group theory and applications. The second author was supported by CMUP (UID/MAT/00144/2019) under FCT research grant IF/00636/2015. The third author was supported by EPSRC standard grant EP/P02310X/1.

Conflict of Interest: The authors certify that they have no affiliations with or involvement in any organization or entity with any financial or non-financial interest in the subject matter or materials discussed in this manuscript.

\section{References}

[1] M. Aschbacher, 'On the maximal subgroups of the finite classical groups', Invent. Math. 76 (1984), 469-514.

[2] M. Aschbacher and R. Guralnick, 'Some applications of the first cohomology group', J. Algebra 90 (1984), 446-460.

[3] M. Aschbacher and L. Scott, 'Maximal subgroups of finite groups', J. Algebra 92 (1985), 44-80.

[4] M. Aschbacher and G. M. Seitz, 'Involutions in Chevalley groups over fields of even order', Nagoya Math. J. 63 (1976), 1-91.

[5] H. Azad, M. Barry and G. Seitz, 'On the structure of parabolic subgroups', Comm. Algebra 18(2) (1990), 551-562.

[6] A. Ballester-Bolinches and L. M. Ezquerro, Classes of Finite Groups, Mathematics and Its Applications (Springer), 584 (Springer, Dordrecht, 2006).

[7] W. Bosma, J. Cannon and C. Playoust, 'The Magma algebra system. I. The user language', J. Symbolic Comput. 24 (1997), 235-265.

[8] N. Bourbaki, Groupes et algèbres de Lie - Chapitres 4,5, et 6, 2nd edn, (Springer, Berlin, Heidelberg, Paris, 1972).

[9] J. N. Bray, D. F. Holt and C. M. Roney-Dougal, The Maximal Subgroups of the LowDimensional Finite Classical Groups, Lecture Note Series, 407 (London Mathematical Society, Cambridge, 2013). 
[10] T. Burness, M. Liebeck and A. Shalev, 'Generation and random generation: From simple groups to maximal subgroups', Adv. Math. 248 (2013), 59-95.

[11] P. Cameron, A. Lucchini and C. Roney-Dougal, 'Generating sets of finite groups', Trans. Amer. Math. Soc. 370(9) (2018), 6751-6770.

[12] R. Carter, 'Centralizers of semisimple elements in finite groups of Lie type', Proc. Lond. Math. Soc. 37 (1978), 491-507.

[13] A. Cohen, M. Liebeck, J. Saxl and G. Seitz, 'The local maximal subgroups of the exceptional groups of Lie type, finite and algebraic', Proc. Lond. Math. Soc. 64(3) (1992), 21-48.

[14] T. Connor and D. Leemans, 'An atlas of subgroup lattices of finite almost simple groups', Ars Math. Contemp. 8 (2015), 259-266.

[15] J. Conway, R. Curtis, S. Norton, R. Parker and R. Wilson, Atlas of Finite Groups, (Oxford University Press, Eynsham, 1985).

[16] C. W. Curtis and I. Reiner, Representation Theory of Finite Groups and Associative Algebras, (AMS Chelsea Publishing, Providence, RI, 2006), Reprint of the 1962 original.

[17] F. Dalla Volta and A. Lucchini, 'Generation of almost simple groups', J. Algebra 178 (1995), 194-223.

[18] F. Dalla Volta and A. Lucchini, 'Finite groups that need more generators than any proper quotient', J. Aust. Math. Soc. 64 (1998), 82-91.

[19] F. Dalla Volta, A. Lucchini and F. Morini, 'On the probability of generating a minimal $d$ generated group', J. Aust. Math. Soc. 71 (2001), 177-185.

[20] E. Detomi and A. Lucchini, 'Crowns and factorization of the probabilistic zeta function of a finite group', J. Algebra 265 (2003), 651-668.

[21] E. Detomi and A. Lucchini, 'Probabilistic generation of finite groups with a unique minimal normal subgroup', J. Lond. Math. Soc. 87 (2013), 689-706.

[22] D. Flaass, '2-local subgroups of Fischer's groups', Mat. Zametki 35 (1984), 333-342.

[23] P. Förster, 'Chief factors, crowns, and the generalized Jordan-Hölder theorem', Comm. Algebra 16 (1988), 1627-1638.

[24] W. Gaschütz, 'Praefrattinigruppen', Arch. Math. 13 (1962), 418-426.

[25] D. Gorenstein and R. Lyons, 'The local structure of finite groups of characteristic 2 type', Mem. Amer. Math. Soc. 42 (1983), 1-731.

[26] D. Gorenstein, R. Lyons and R. Solomon, The Classification of the Finite Simple Groups Number Three, Mathematical Surveys and Monographs, 40 (3) (American Mathematical Society, Providence, 1991).

[27] P. E. Holmes and R. A. Wilson, 'A new computer construction of the Monster using 2-local subgroups', J. Lond. Math. Soc. 67 (2003), 349-364.

[28] J. Humphreys, Linear Algebraic Groups, (Springer, New York, 1975).

[29] P. Jiménez-Seral and J. P. Lafuente, 'On complemented non-abelian chief factors of a finite group', Israel J. Math. 106 (1998), 177-188.

[30] P. Kleidman, 'The maximal subgroups of the finite 8-dimensional orthogonal groups $\mathrm{P} \Omega_{8}^{+}(q)$ and of their automorphism groups', J. Algebra 110 (1987), 173-242.

[31] P. Kleidman and M. Liebeck, The Subgroup Structure of the Finite Classical Groups, London Mathematical Society Lecture Note Series, 129 (Cambridge University Press, 1990).

[32] P. Kleidman, R. Parker and R. Wilson, 'The maximal subgroups of the Fisher group $F i_{23}$ ', J. Lond. Math. Soc. 39 (1989), 89-101.

[33] P. Kleidman and R. Wilson, 'The maximal subgroups of $\mathrm{Fi}_{22}$ ', Math. Proc. Cambridge Philos. Soc. 102 (1987), 17-23.

[34] M. Liebeck, J. Saxl and G. Seitz, 'Subgroups of maximal rank in finite exceptional groups of Lie type', Proc. Lond. Math. Soc. (3) 65 (1992), 297-325. 
[35] M. Liebeck and G. Seitz, 'Maximal subgroups of exceptional groups of Lie type, finite and algebraic', Geom. Dedicata 35(1-3) (1990), 353-387.

[36] S. Linton and R. Wilson, 'The maximal subgroups of the Fischer groups $F i_{24}$ and $F i_{24}^{\prime}$ ', Proc. Lond. Math. Soc. 63 (1991), 113-164.

[37] A. Lucchini and F. Menegazzo, 'Generators for finite groups with a unique minimal normal subgroup', Rend. Semin. Mat. Univ. Padova 98 (1997), 173-191.

[38] A. Lucchini and F. Morini, 'On the probability of generating finite groups with a unique minimal normal subgroup', Pac. J. Math. 203 (2002), 429-440.

[39] R. Lyndon and P. Schupp, Combinatorial Group Theory (Springer, Berlin, 1977).

[40] G. Malle and D. Testerman, Linear Algebraic Groups and Finite Groups of Lie Type, Cambridge Studies in Advanced Mathematics, 133 (Cambridge University Press, Cambridge, 2011).

[41] U. Meierfrankenfeld and S. V. Shpectorov, 'Maximal 2-local subgroups of the Monster and Baby Monster', Preprint, 2002. Michigan State University, Available from http://www. mth.msu.edu/meier/Preprints/2monster/maxmon.pdf.

[42] N. Menezes, M. Quick and C. Roney-Dougal, 'The probability of generating a finite simple group', Israel J. Math. 198(1) (2013), 371-392.

[43] G. Miller, 'On the groups generated by two operators', Bull. Amer. Math. Soc. 7 (1901), 424-426.

[44] S. Norton and R. Wilson, 'Maximal subgroups of the Harada-Norton Group', J. Algebra 103 (1986), 362-376.

[45] S. Norton and R. Wilson, 'A correction to the 41-structure of the Monster, a construction of a new maximal subgroup $\mathrm{L}_{2}(41)$ and a new Moonshine phenomenon', J. Lond. Math. Soc. 87 (2013), 943-962.

[46] G. Seitz, 'Generation of finite groups of Lie type', Trans. Amer. Math. Soc. 271 (1982), 351-407.

[47] R. Steinberg, 'Generators for simple groups', Canad. J. Math. 14 (1962), 277-283.

[48] R. Wilson, 'The maximal subgroups of Conway's group Coo ', J. Algebra 85 (1983), 144-165.

[49] R. Wilson, 'Some subgroups of the Baby Monster', Invent. Math. 89 (1987), 197-218.

[50] R. Wilson, 'The local subgroups of the Fischer groups', J. Lond. Math. Soc. (2) 36 (1987), 77-94.

[51] R. Wilson, 'The maximal subgroups of the Baby Monster, I', J. Algebra 211 (1999), 1-14.

[52] R. Wilson, R. Parker, S. Nickerson, T. Breuer and J. Bray, 'AtlasRep, A GAP Interface to the Atlas of Group Representations', Version 1.5.1 (2016), http://www.math.rwth-aachen.de/ $\sim$ Thomas.Breuer/atlasrep.

[53] R. Wilson et al., 'A World-Wide-Web Atlas of finite group representations', http://brauer.maths.qmul.ac.uk/Atlas/v3/. 